\title{
The role of human biological monitoring in health risk assessment
}

\author{
Roma Gurusankar* \\ BMK Scientific Consultancy, \\ Ottawa, Ontario, Canada, \\ Email: romagurusankar@hotmail.com \\ *Corresponding author
}

\section{Nagarajkumar Yenugadhati}

University of Ottawa,

McLaughlin Centre for Population Health Risk Assessment,

Ottawa, Ontario, Canada

Email: nagaraj.yenugadhati@uottawa.ca

\section{Kannan Krishnan}

Département de santé environnementale et santé au travail, Université de Montréal,

Montréal, Quebec, Canada

Email: kannan.krishnan@umontreal.ca

\section{Sean Hays}

Summit Toxicology, LLP,

Allenspark, Colorado, USA

Email: shays@summittoxicology.com

\section{Douglas Haines}

Healthy Environments and Consumer Safety Branch, Health Canada,

Ottawa, Ontario, Canada

Email: doug.haines@hc-sc.gc.ca

\section{Angelika Zidek and Sandra Kuchta}

Assessment Methodology Division,

Health Canada,

Ottawa, Ontario, Canada

Email: angelika.zidek@hc-sc.gc.ca

Email: sandra.kuchta@hc-sc.gc.ca 


\title{
David Kinniburgh
}

University of Calgary,

Alberta Centre for Toxicology,

Alberta, Canada

Email: dkinnibu@ucalgary.ca

\section{Stephan Gabos}

Department of Laboratory Medicine and Pathology,

Division of Analytical and Environmental Toxicology,

University of Alberta,

Alberta, Canada

Email:sgabos@ualberta.ca

\section{Donald Mattison and Daniel Krewski}

University of Ottawa,

McLaughlin Centre for Population Health Risk Assessment, Ottawa, Ontario, Canada

and

Risk Sciences International,

Ottawa, Ontario, Canada

Email: dmattison@risksciences.ca

Email: dkrewski@uottawa.ca

\begin{abstract}
Human biological monitoring refers to the measurement of biomarkers in biological specimens. Advances in analytical chemistry together with an increased understanding of the potential toxicity of environmental chemicals have propelled the quest to identify and monitor chemicals and metabolites in human biological specimens. Many biomonitoring programs have provided valuable data on the presence of environmental chemicals in biological matrices. The availability of this large volume of biomonitoring data has increased the need to understand this information with respect to potential human health risks. This review summarises approaches for interpreting biomonitoring data in the context of population health and risk assessment. Moreover, the advantages and limitations of human biomonitoring approaches, major challenges in the interpretation of HBM data and the utility of human biomonitoring data in health risk assessment context are presented. Several knowledge gaps to improve the ability to interpret human biomonitoring data are discussed.
\end{abstract}

Keywords: human biomonitoring; interpretation of biomonitoring data; forward dosimetry; biomonitoring equivalents; HBM-I; HBM-II; reverse dosimetry; daily intake estimation; triclosan; phthalates.

Reference to this paper should be made as follows: Gurusankar, R., Yenugadhati, N., Krishnan, K., Hays, S., Haines, D., Zidek, A., Kuchta, S., Kinniburgh, D., Gabos, S., Mattison, D. and Krewski, D. (2017) 'The role of human biological monitoring in health risk assessment', Int. J. Risk Assessment and Management, Vol. 20, Nos. 1/2/3, pp.136-197. 
Biographical notes: Roma Gurusankar is the proprietor of BMK Scientific Consultancy, a toxicology consulting firm in Ottawa, Ontario. She has previously held Natural Sciences and Engineering Research Council of Canada Visiting Fellowship at the Inhalation Toxicology Laboratory, Health Canada, Ottawa, Ontario, and has worked as a Research Associate at Risk Sciences International Inc., Ottawa, Ontario. Her areas of expertise include toxicological evaluation and interpretation of health effects due to low-level ambient air pollutants, conducting human biomonitoring studies, interpretation of human biomonitoring data, risk assessment of chemical mixtures and development of toxicity profiles of environmental pollutants and microbes.

Nagarajkumar Yenugadhatii s an Epidemiologist at University of Ottawa. His academic background includes a Master's in Epidemiology and a Bachelor's in Medicine. His research interests are in epidemiology, biostatistics, health risk assessment and management, systematic reviews, toxicology and post market drug surveillance studies. In addition, heprovides his expertise in conducting statistical analysis using SAS and other analytical software to scientific committees and research centres.

Kannan Krishnan is a Professor and acting Director at the Department of Occupational and Environmental Health of the University of Montreal (Canada) where he also serves as the Vice-Dean of Research at the School of Public Health. $\mathrm{He}$ is a Board-Certified Toxicologist (Diplomate of the American Board of Toxicology) and elected as the Fellow of both the Academy of Toxicological Sciences and the Canadian Academy of Health Sciences. His key research contributions are in the areas of PBPK modelling, chemical mixtures, risk assessment methods and biomonitoring equivalents of environmental chemicals.

Sean Hays is the President and the Founder of Summit Toxicology, a toxicology and risk assessment consulting firm headquartered in Colorado. $\mathrm{He}$ is an Assistant Clinical Professor at the University of Colorado, School of Public Health, and an Affiliate Faculty in Chemical Engineering at Colorado State University. His expertise is in pharmacokinetic modelling and the interpretation of human biomonitoring data.

Douglas Haines is a Senior Science Advisor in Health Canada's Healthy Environments and Consumer Safety Branch. Under the Government of Canada's Chemicals Management Plan, he has developed and implemented national human biomonitoring and environmental monitoring initiatives to track Canadians' exposures to environmental chemicals and their integration into health risk assessments. This includes the Canadian Heath Measures Survey which was launched in 2007 and is providing biomonitoring and human health data on a national scale and surveillance in Canada's north which is carried out in partnership with the federal Northern Contaminants Program.

Angelika Zidek has worked in the field of exposure, risk assessment and pollution abatement for over 15 years and is currently a Manager at Health Canada's Existing Substances Risk Assessment Bureau. Her experience includes occupational and general population exposure to pesticides and industrial chemicals, waste management, as well as environmental compliance programs. As a Canadian delegate on the OECD Exposure Assessment Task Force, she works with other OECD member countries on ways to share tools and information related to environmental and human health exposure as well as opportunities to harmonise and collaborate on approaches related to chemical risk assessment. 
Sandra Kuchta is a Scientific Evaluator in the Existing Substances Risk Assessment Bureau at Health Canada. She works on the risk assessment of chemicals under Canada's Chemical Management Plan (CMP). Her academic background includes a Master of Science in Toxicology and a Bachelor of Science in Chemistry.

David Kinniburgh is the Director of the Alberta Centre for Toxicology at the University of Calgary and the Principal Investigator for the Alberta Biomonitoring Project. $\mathrm{He}$ is an Adjunct Associate Professor with the Department of Physiology and Pharmacology, Cumming School of Medicine, at the University of Calgary and a Clinical Professor with the Department of Laboratory Medicine and Pathology at the University of Alberta. He is a Certified Clinical Biochemist (American Board of Clinical Chemistry) and a Fellow of the Canadian Academy of Clinical Biochemistry. His research interests include clinical and environmental toxicology.

Stephan Gabos graduated from the University of Medicine and Pharmacy, Tirgu Mures, Romania. He started his carrier as a pathologist and later he trained in epidemiology and health information management. He was employed in various research, administrative, and executive management positions with the Cancer Board and the Ministry of Health in Alberta, Canada. He retired from the government in 2013 and continues to work in the academia as Research Scientist in the Division of Analytical and Environmental Toxicology, University of Alberta. He has a broad range of scientific research interests including environmental toxicology and epidemiology, cytotoxicity, exposure assessment, biomonitoring, and health risk assessment. For his service to the Government of Alberta and his contributions in the field of environmental health, he received four times the Alberta Premier's Award of Excellence. In 2015 he was inducted in the organisation The Leading Physicians of the World.

Donald Mattison is the Chief Medical Officer of Risk Sciences International and an Associate Director of the McLaughlin Centre for Population Health Risk Assessment, University of Ottawa. He also serves as the Medical Advisor to QuarterWatch, Institute for Safe Medication Practices and is a Senior Research Fellow of the International Prevention Research Institute. His research interests include reproductive and developmental toxicology, pharmacovigilance and pharmacology, observational research in pharmacoepidemiology, drug safety and efficacy research.

Daniel Krewski is a Professor in the School of Epidemiology, Public Health and Preventive Medicine at the University of Ottawa, where he also serves as Scientific Director of the McLaughlin Centre for Population Health Risk Assessment. His research interests include epidemiology, biostatistics, risk assessment, and risk management. He is a Fellow of the Society for Risk Analysis, the American Statistical Association, and a National Affiliate of the US National Academy of Sciences. He holds the Natural Sciences and Engineering Research Council of Canada Chair in Risk Science at the University of Ottawa.

This paper is a revised and expanded version of a paper entitled 'Canada health measures survey' presented at Health Canada Training Seminar on Risk Science in the 21st Century, University of Ottawa, Ottawa, Canada, 4-6 March 2013. 


\section{Introduction}

Human biological monitoring or biomonitoring (HBM) is measurement of the concentration of biomarker(s) (chemicals, metabolites and/or products formed due the interaction of the chemicals with the body) in biological specimens such as blood, urine, saliva, breast milk, meconium, toe nails, and hair. HBM provides a direct measure of the internal dose of chemical(s) and is reflective of exposures and disposition (including absorption, distribution, metabolism and elimination) from all exposures (inhalation, ingestion, dermal exposure, pre-natal exposure) (NRC, 2006; Angerer et al., 2007; Needham et al., 2007; Clewell et al., 2008). Biomarkers (and hence biomonitoring) can be broadly classified into exposure (e.g., measurement of metals such as lead in blood), biochemical effects (e.g., acrylamide adducts of hemoglobin) and biological effects (e.g., plasma acetyl cholinesterase activity) based on their functional characteristics and the field of application (NRC, 2006). Because HBM can characterise exposure, biochemical and biological effects they are of great interest in population health.

\section{Role of biomonitoring in protecting human health}

Human exposure to environmental chemicals occurs from multiple sources and, as depicted in Figure 1, can enter the body (absorption) through multiple routes (e.g., ingestion, inhalation and dermal contact). After absorption (producing an internal dose), chemicals undergo distribution, metabolism and excretion. Throughout these steps (defined as pharmacokinetics (PKs), or what the body does to the chemical) the chemical and/or its metabolites reach the target site(s) to elicit health effects. In traditional human health risk assessment, the exposure-health effect continuum depicted in Figure 1 is broken down into various steps, for example exposure assessment (quantification of exposure dose of a chemical), toxicity assessment (development of dose-response relationship of the chemical to derive guidance values) and risk characterisation (traditionally the product of exposure and substance guidance value). However, with HBM it is possible to compare the HBM concentrations to guidance values to quantify risk. Although the approaches and methods used in the risk assessment of carcinogenic and non-carcinogenic chemicals differ considerably, the basic principles remain the same. The data from HBM studies help to reduce the uncertainties in exposure assessment and provide valuable data to link the exposure to health effects (Angerer, 2012). Several aspects of human biomonitoring are explained by the National Research Council in its report on human biomonitoring of environmental chemicals in 2006 (NRC, 2006) and emphasised the importance of biomonitoring in exposure sciences in its report, Exposure Science in the 21st Century in 2012 (NRC, 2012).

Some of the major benefits of HBM include:

- detection of reliable biomarker(s) of a given chemical in an appropriate biological specimen provides direct evidence that exposure has occurred

- chemical-specific biomarker concentration measured in biospecimens is proportional to the internal dose of the chemical (i.e., body burden) and provides an integrated estimate of exposures from all sources and pathways 
- HBM can be used in surveillance programs to measure the concentrations of chemicals in the general population, identify vulnerable populations and sub-populations with higher than normal exposure to chemicals

- HBM can also be used to develop baseline concentrations of chemicals in a given population and assess the time trends

- as it provides a measure of internal dose, HBM has the potential to reduce the uncertainty of exposure characterisation in risk assessments

- through HBM it is possible to gather evidence for the effectiveness of any risk management measures that were implemented to reduce population exposure to chemicals

- HBM can be used to test research hypotheses, identify knowledge gaps and initiate new research pertaining to exposure sources, pathways and health effects (Albertini et al., 2006; NRC, 2012).

Figure 1 The forward and reverse dosimetry approaches for evaluating human biomonitoring data within a population health risk assessment context

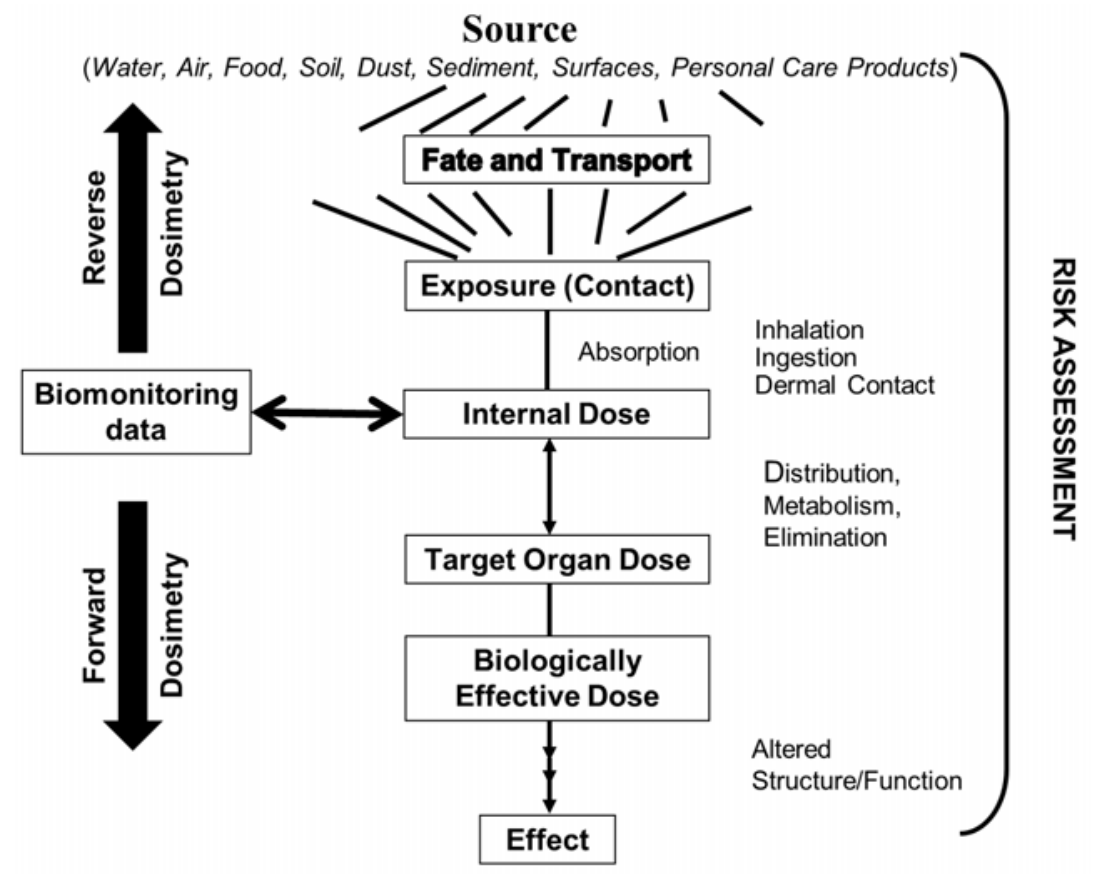

Source: Figure modified from Angerer et al. (2006) 
Like any other scientific approach, HBM has limitations that require special attention when interpreting biomonitoring data (Smolders et al., 2009). These limitations include:

- Non-detection of a biomarker in an individual does not necessarily mean that he/she is not exposed to a given chemical. This is because the biomarker detection in the biological specimen depends on several factors including the sensitivity and specificity of the biomarker to reflect exposure to the chemical of concern, availability of validated analytical method(s), physicochemical characteristics of the chemical, its PKs, the frequency of exposure and the timing between the exposure and sampling.

- At the same time, detection of a chemical in an individual does not necessarily mean that health effects would occur. Additional information regarding PKs as well as toxicity of the chemicals is essential to relate the biomonitoring data to potential health effects (Albertini et al., 2006).

- Moreover, since HBM data provides an integrated estimate of exposure, complementary information on exposure sources and pathways are required to develop possible population health interventions (Clewell et al., 2008).

HBM has played an important role in protecting the health of the general population for selected exposures. For example, biomonitoring of blood lead levels over several decades has been used successfully to assess lead exposure and health consequences in the general public and helped in developing health policies to reduce the exposure (CDC, 2005; Bushnik et al., 2010; Health Canada, 2013a). Moreover, HBM has been used to ascertain the effectiveness of public policies, for example related to second hand smoke, by demonstrating a decline in blood cotinine levels in children (CDC, 2010).

\section{Major biomonitoring programs in the world}

The availability of new analytical methods and their ability to detect chemicals in biological specimens at very low concentrations has increased the relevance of HBM data to human health risk assessment. Currently, several countries, including Canada, have ongoing biomonitoring programs to evaluate exposure to chemicals in the general population (Table 1). Although not exhaustive, the biomonitoring programs summarised in Table 1 provide a glimpse of diverse types of HBM activities conducted around the world. The list also illustrates the diverse population groups included (nationally representative populations, pregnant women, children, etc.) as well as different groups of chemicals measured in these surveys.

These HBM surveys have increased public awareness about the presence of many chemicals in humans (Sexton et al., 2004; Joas et al., 2014). Consequently, there is an increased demand to interpret the findings from biomonitoring surveys in the population health context. Important features of some of national and international biomonitoring programs are summarised in Table 1. 
Table 1 List of population-level biomonitoring programs in Canada and around the world

\begin{tabular}{|c|c|c|c|c|}
\hline 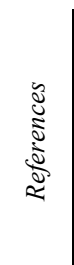 & 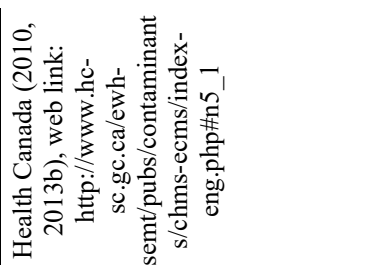 & 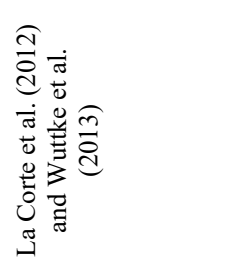 & 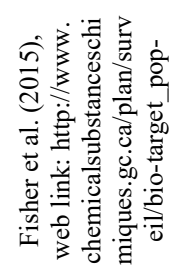 & 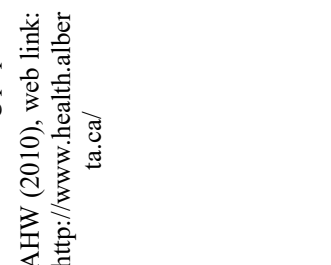 \\
\hline 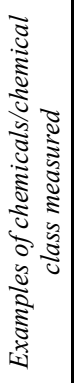 & 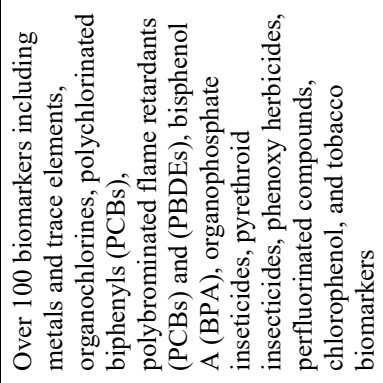 & 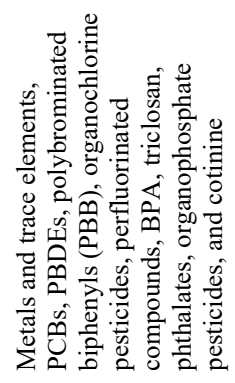 & 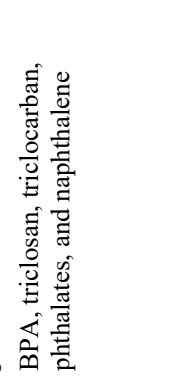 & 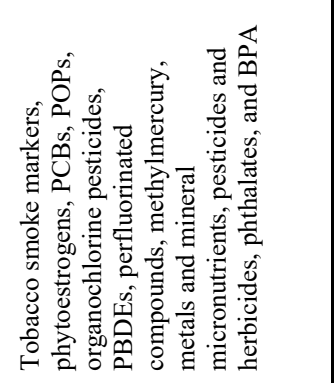 \\
\hline 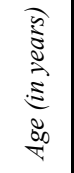 & îj & $\stackrel{\stackrel{\sim}{\wedge}}{\wedge}$ & $\stackrel{\infty}{\wedge \prime}$ & $\begin{array}{l}m \\
\text { b } \\
\text { 产 } \\
\text { ज. } \\
\text { ni } \\
\text { vi }\end{array}$ \\
\hline & 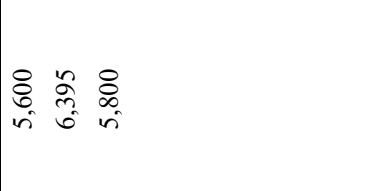 & 总 & 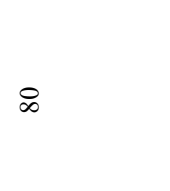 & 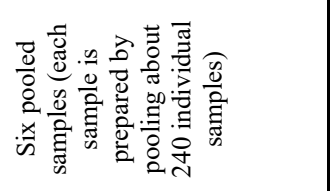 \\
\hline ఫ్ & 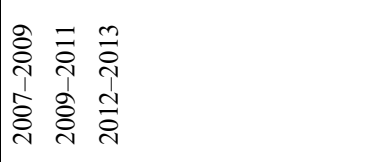 & $\overline{\vec{N}}$ & 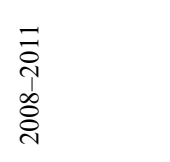 & 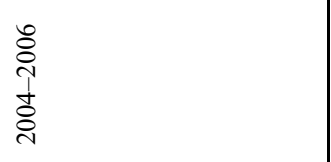 \\
\hline 总 & 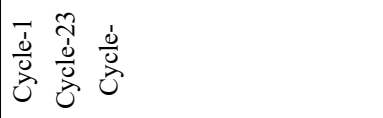 & & & \\
\hline $\begin{array}{l}\text { o } \\
\vdots \\
0 \\
0 \\
0 \\
0\end{array}$ & 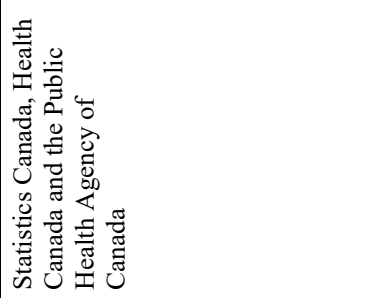 & 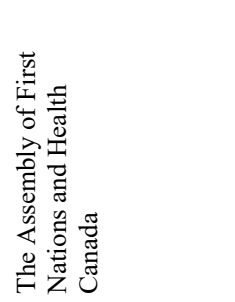 & 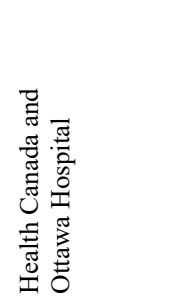 & 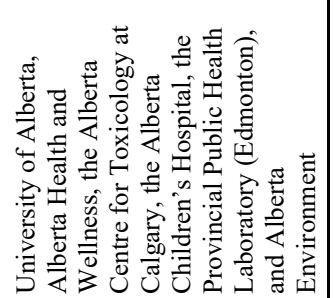 \\
\hline ڤั & 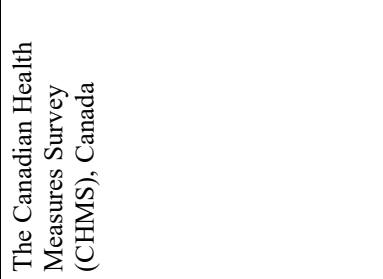 & 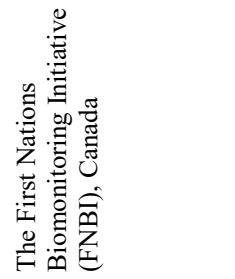 & 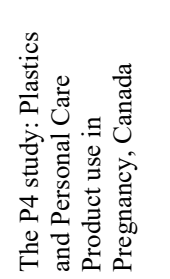 & 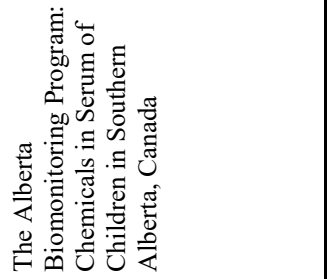 \\
\hline
\end{tabular}


Table 1 List of population-level biomonitoring programs in Canada and around the world (continued)

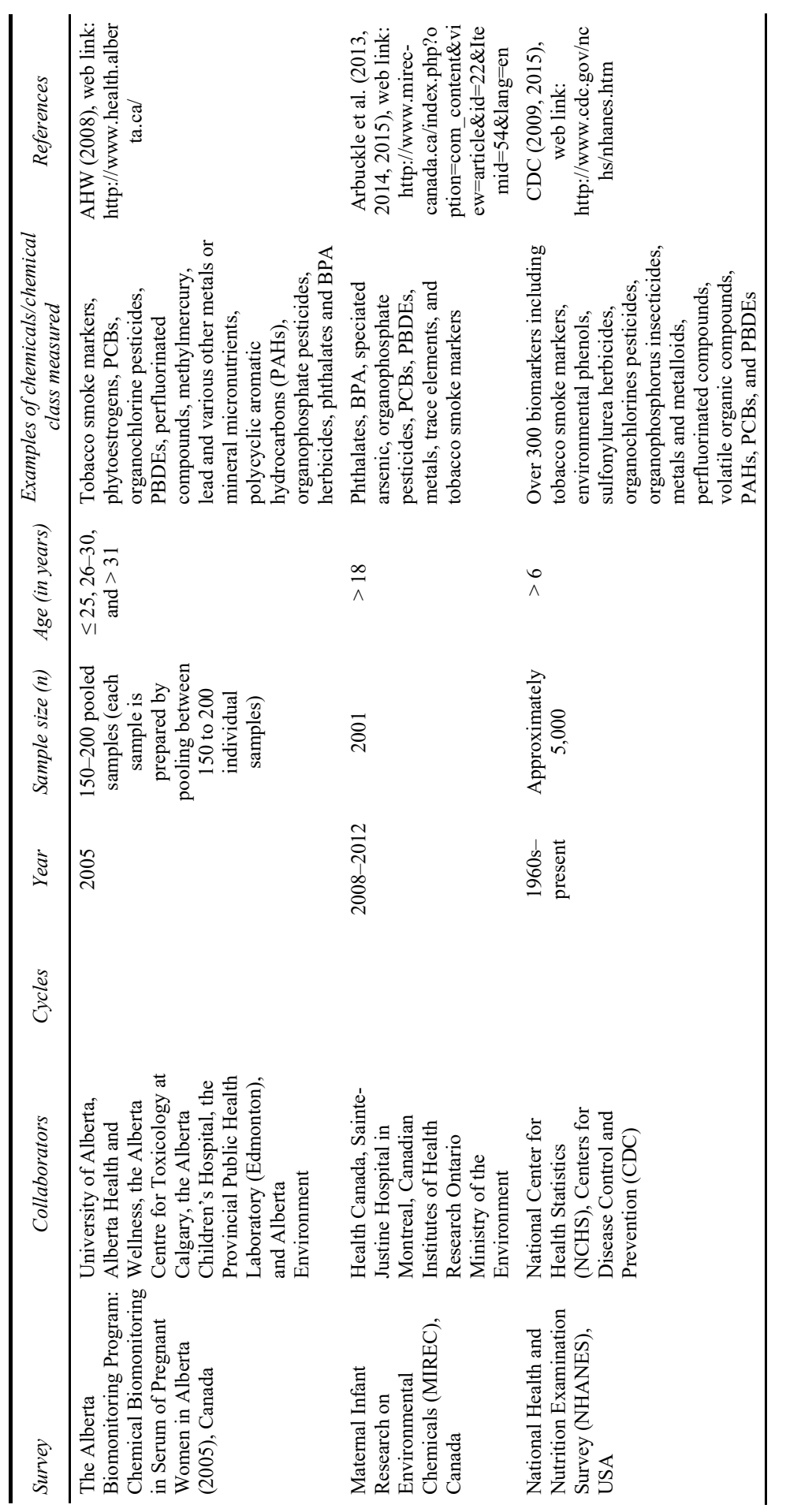


Table 1 List of population-level biomonitoring programs in Canada and around the world (continued)

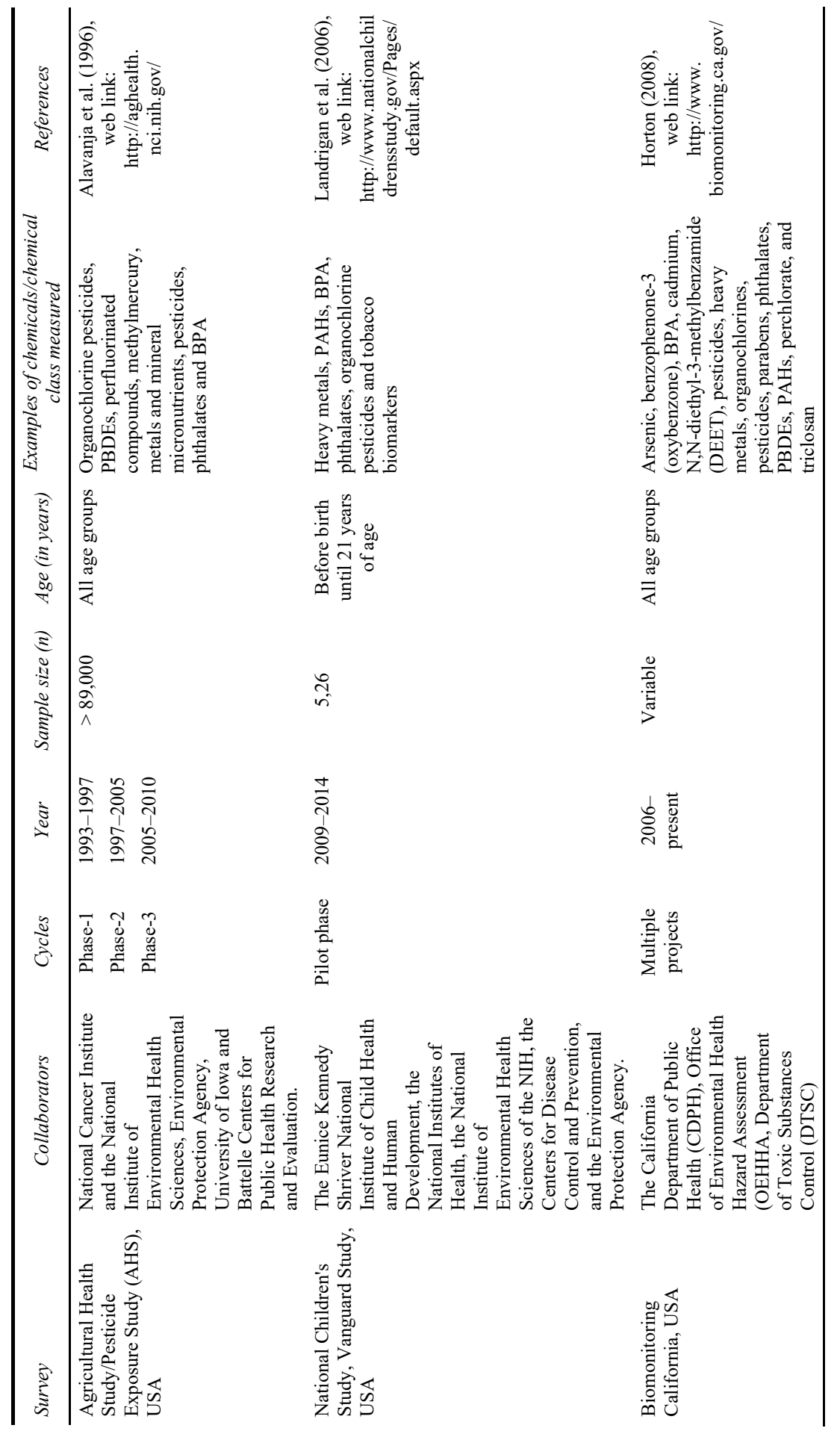


Table 1 List of population-level biomonitoring programs in Canada and around the world (continued)

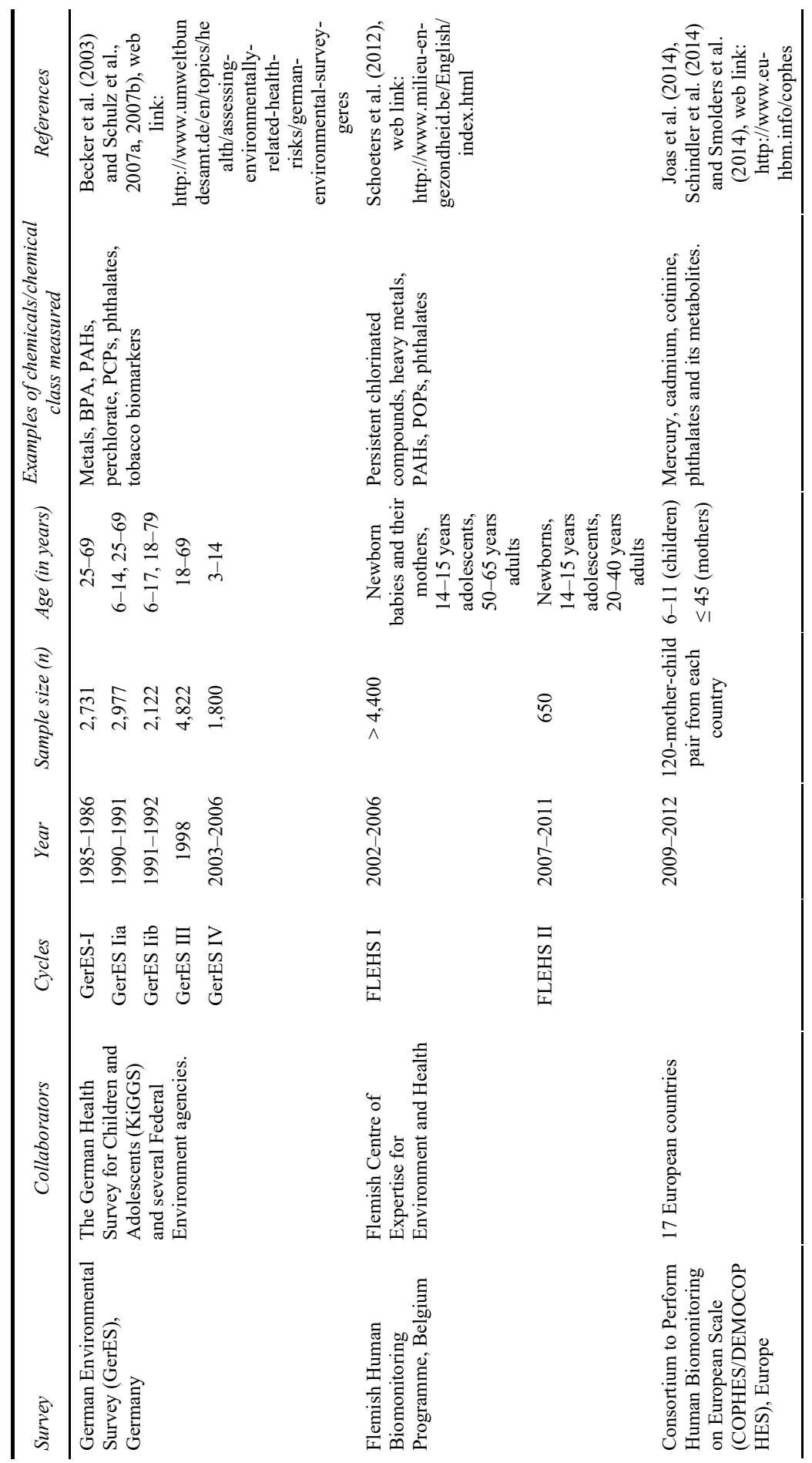


Table 1 List of population-level biomonitoring programs in Canada and around the world (continued)

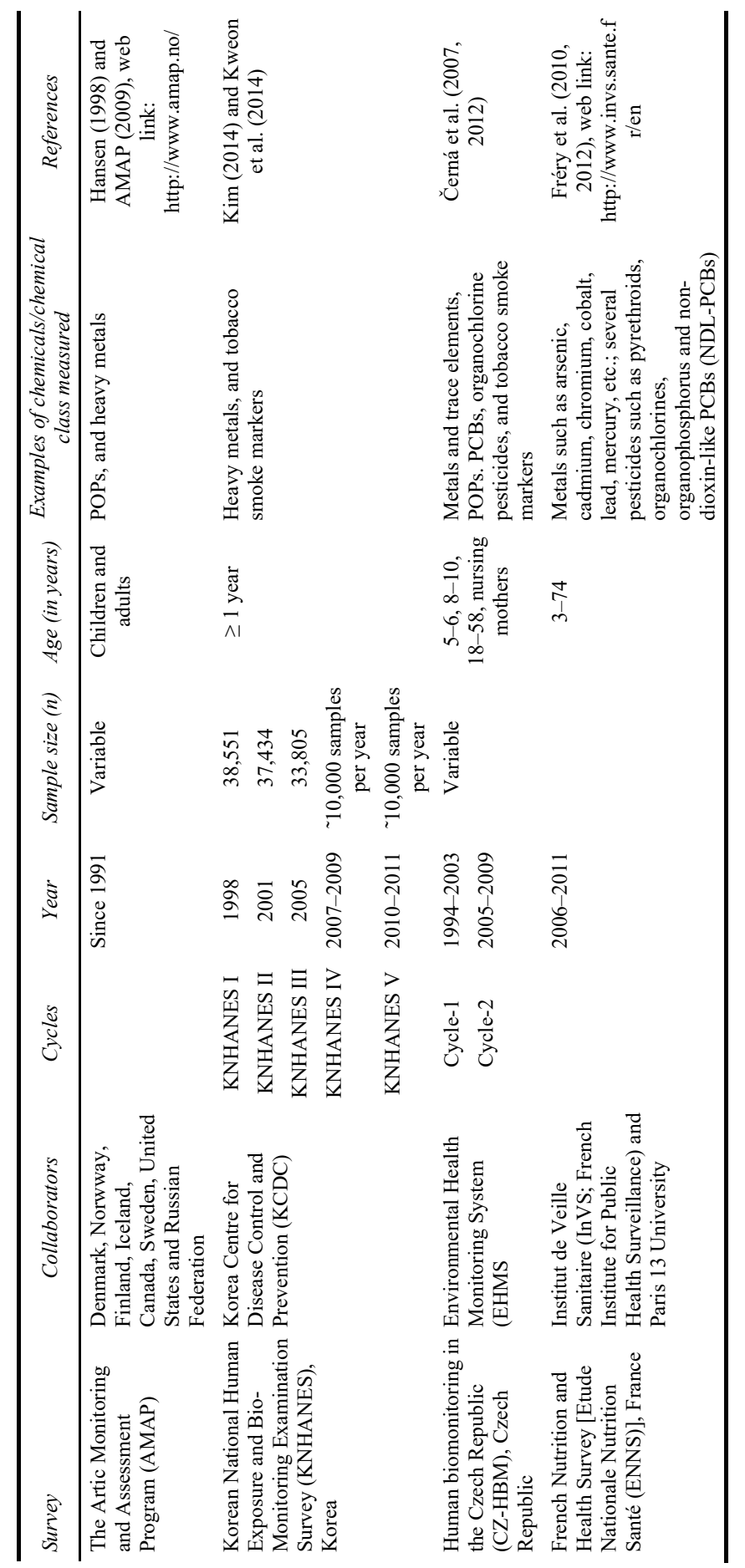




\subsection{The Canadian Health Measures Survey (CHMS), Canada}

The Canadian Health Measures Survey (CHMS) is a nationally representative comprehensive health measures survey carried out by Statistics Canada in collaboration with Health Canada and the Public Health Agency of Canada. It collects information on the general health and lifestyles of Canadians through interviews and direct physical measurements. This ongoing survey is conducted in two-year cycles and includes a biomonitoring component. The survey measures the environmental exposure of Canadians to chemicals, both natural as well as synthetic (http://www.hc-sc.gc.ca/ewhsemt/pubs/contaminants/chms-ecms/index-eng.php\#n5_1). In cycle 1 (2007-2009) approximately 5,600 Canadians aged 6-79 years and from 15 sites across Canada participated (Health Canada, 2010). Cycle 2 (2009-2011) included 6,395 Canadians aged 3-79 years from 18 sites across Canada (Health Canada, 2013b). In cycle 3 (2012-2013) approximately 5,800 Canadians ranging from 3-79 years of age, from 16 sites across Canada participated. Field work for cycle 4 began in 2014.

The first stage of each cycle is a household interview, where the participants undergo a personal interview and fill in a questionnaire. In the second stage, participants visit the CHMS mobile examination centre (MEC) for physical measurements. Blood and urine samples are collected from the participants by trained heathcare workers at the MEC. Several chemical contaminants are measured in the biological samples (blood and urine) in private and government analytical laboratories within Canada. In addition, presence of chemical contaminants in the household indoor air (cycles 2 and 3) and tap water (cycles 3 and 4) were also measured.

The main objective of the CHMS is to provide human biomonitoring data to scientists, and health and environment officials in order to:
“...establish reference ranges for concentrations of chemicals in Canadians, which could allow for comparisons with subpopulations in Canada and comparisons with other countries; establish baseline levels of chemicals to track trends of exposure levels in Canadians over time; to provide information for setting priorities and taking action to protect the health of Canadians and to protect Canadians from exposure to environmental chemicals; assess the effectiveness of regulatory and environmental risk management actions intended to reduce exposures and health risks from specific chemicals; to support future research on potential links between exposure to certain chemicals and specific health effects; and, to contribute to international monitoring programs, such as the Stockholm Convention on Persistent Organic Pollutants." (Health Canada, 2010)

Several classes of environmental chemicals were measured in the CHMS including metals and trace elements, organochlorines, polychlorinated biphenyls (PCBs), polybrominated flame retardants (PBDEs), benzene, phthalates, environmental phenols (bisphenol A, triclosan, triclocarban), organophosphate insecticides, pyrethroid insecticides, atrazine and carbamate pesticides, phenoxy herbicides, perfluorinated compounds, chlorophenol, polycyclic aromatic hydrocarbons, and tobacco (cotinine as the primary metabolite of nicotine).

\subsection{The First Nations Biomonitoring Initiative (FNBI), Canada}

The Assembly of First Nations (AFN), in partnership with Health Canada (through the First Nations and Inuit Health Branch, FNIHB), carried out a HBM program, The First 
Nations Biomonitoring Initiative (FNBI), to establish baseline information on human exposure to environmental chemicals for First Nations people living on reserves. While the CHMS represents the Canadian population, it does not include First Nation peoples living on reserves. There is no nationally representative information about the presence as well as the concentrations of environmental chemicals in First Nation populations. The FNBI was implemented to fill this knowledge gap (La Corte and Wuttke, 2012; Wuttke et al., 2013).

Data were collected from individuals across 13 randomly selected First Nation communities $(n=503), \geq 20$ years of age, during summer/fall of 2011 . The participants underwent a physical examination (i.e., height and weight measurement), filled out a household questionnaire and provided a biological specimen of blood and urine.

The following environmental chemicals or their metabolites were measured: metals and trace elements, PCBs, polybrominated diphenyl ethers and polybrominated biphenyls, organochlorine pesticides, perfluorinated compounds, environmental phenol, phthalates, pesticides, and cotinine. These were compared to concentrations in the Canadian general population using CHMS data, identifying similarities and differences in concentrations between these populations.

\title{
3.3 Maternal-Infant Research on Environmental Chemicals (MIREC) study, Canada
}

Maternal-Infant Research on Environmental Chemicals (MIREC) is a national level five-year biomonitoring study conducted on about 2,000 women recruited from Vancouver, Edmonton, Winnipeg, Sudbury, Ottawa, Kingston, Hamilton, Toronto, Montreal and Halifax, during the first trimester of pregnancy and followed through the pregnancy and up to eight weeks after birth. The participants had to be 18 years of age or older and 6 to 12 weeks pregnant (Arbuckle et al., 2013).

The main goals of this study are:

\begin{abstract}
"to measure the extent to which pregnant women and their babies are exposed to environmental chemicals, as well as tobacco smoke; to assess what pregnancy health risks, if any, are associated with exposure to heavy metals (lead, mercury, cadmium, arsenic and manganese); to measure the levels of environmental chemicals and some of the beneficial components (nutritional and immune constituents) of breast milk." (http://www.hc-sc.gc.ca/ewhsemt/contaminants/human-humaine/mirec-eng.php)
\end{abstract}

The MIREC study is a collaborative effort of Health Canada scientists, the Sainte-Justine Hospital in Montreal, and clinical researchers from the other participating cities. Sainte-Justine Hospital in Montreal is the coordinating centre for the study. MIREC is co-funded by Health Canada, the Canadian Institutes of Health Research (CIHR) and the Ontario Ministry of Environment. Biomarkers of environmental contaminants such as phthalates, bisphenol A, speciated arsenic, organophosphate pesticides, creatinine, PCBs, metals, trace elements, polybrominated diphenylethers (PBDEs), triclosan, and tobacco smoke exposure (e.g., cotinine) were measured in the biological samples such as mothers' blood, urine, hair, breast milk, umbilical cord blood and meconium (Arbuckle et al., 2014, 2015). A biobank was created to maintain the remaining unused portion of the biospecimens. In addition to environmental contaminants, the biospecimens were also analysed for susceptibility/effects biomarkers such as endothelins, and oxidative stress biomarkers (e.g., o-tyrosine). 
To study the effect of prenatal exposure to environmental chemicals on the growth and development of infants, a follow up study, Maternal-Infant Research on Environmental Chemicals-Infant Development (MIREC-ID), was initiated in 2009. In this follow-up study babies born to a subset of MIREC mothers $(n=525)$ were assessed for parameters pertaining to child development at birth and at 6 months of age (http://www.mirec-canada.ca). This study was completed in 2012 and the results are being analysed. Subsequently, other follow-up studies to assess the development of children born to MIREC mothers at age three, [Maternal-Infant Research on Environmental Chemicals-Child Development at 3; MIREC-CD3], and at ages two and five years, (Maternal-Infant Research on Environmental Chemicals-Child Development plus; MIREC-CDplus), are planned. These studies will characterise the population health impact of a broad set of environmental chemicals on maternal, pregnancy and infant health in Canada.

\subsection{The Alberta biomonitoring program}

In 2005, Alberta Health and Wellness (AHW), now called Alberta Health, along with the University of Alberta, Edmonton, the Alberta Centre for Toxicology (ACFT, University of Calgary) at Calgary, and the ProvLab, Edmonton, conducted a biomonitoring program in pregnant women with the objective to determine the priority environmental contaminants in their blood (AHW, 2008). The specific objectives of the study were to assess the influence of age, geographic location and seasonality in maternal serum concentrations of environmental contaminants. The priority chemicals were selected based on expert guidance and review of similar studies in other countries, such as US Centers for Disease Control and Prevention (CDC). Some emerging chemicals of interest were also included (e.g., Bisphenol A). About 60 chemicals were finally analysed: organochlorines, polybrominated compounds, perfluorinated compounds, methylmercury, metals and mineral micronutrients, and phenolic compounds.

Serum samples of pregnant women were selected from 50,599 serum samples that had been submitted to the ProvLab in Edmonton, in 2005 for screening of infectious diseases in early pregnancy. Individual samples were not analysed, but the residual samples containing at least $1 \mathrm{ml}$ of sample were pooled for analytical purposes. A total of 28,484 samples were selected randomly from 44,584 samples stratified on three geographic regions in Alberta (northern, central and southern regions), three age groups $(\leq 25,26-30$, and above 31 years) and month of receipt, and pooled to obtain at least $150 \mathrm{ml}$ sample for chemical analysis. The number of replicate pools generated varied among geographic region and age strata ranging from 8 to 24 pools. In addition, seven bovine serum control pools were obtained to account for any contamination during handling and chemical analysis. Temporal variations were considered only for Southern Alberta due to data limitations such as availability of sufficient pooled samples. Weighted regression analysis was performed on the data owing to the variations in the replicate pools within geographic regions.

Between 2004 and 2006, AHW and ACFT carried out a follow up study focusing on children. The same set of biomarkers measured in the pregnant women study was evaluated in serum samples to allow comparison (AHW, 2008, 2010).

Small serum samples $(400 \mu \mathrm{L})$ were drawn from 1,373 stored samples at the Alberta Children's Hospital, Calgary that were obtained from healthy children admitted for elective surgeries between 2004 and 2006. The sera were pooled together to obtain three 
replicate pools for each of the two age groups of children $(\leq$ five years and six to 13 years); the number of individual samples in each pool ranged between 196 and 240 samples. For quality assurance purposes seven pools of bovine serum or water were used as controls. The influence of age $(\leq 5,6-13)$ on the concentrations of contaminants was observed, and the levels were compared to the concentration in a previous study in pregnant women (AHW, 2010). These studies are important in characterising maternal pregnancy and childhood exposures and resultant internal concentrations which may influence health and development of two populations thought to be sensitive to chemical exposures.

In 2015, ACFT is completing the recruitment of pregnant woman into a biomonitoring study designed to determine the level of contaminants in mothers and cord blood of newborn babies.

\subsection{National Health and Nutrition Examination Survey (NHANES), USA}

The National Health and Nutrition Examination Survey (NHANES) is designed to assess the health and nutritional status of the American population. NHANES is a major program of the National Center for Health Statistics (NCHS). NCHS is part of the US $\mathrm{CDC}$ and produces statistics on various health parameters for the general American population.

The NHANES program began in the early 1960s and since that time a series of surveys that focus on different population groups or health topics have been conducted. The sample size for each year is about 5,000 people over six years of age and currently operates continuously, with results reported in two-year cycles (e.g., 2009-2010, 2011-2012, etc.). The survey comprises two stages, an interview stage and then an examination stage. The NHANES interview includes a questionnaire that has demographic, socioeconomic, dietary, and health-related questions. The examination component consists of medical, dental, and physiological measurements, administered by highly trained medical personnel.

The fourth national biomonitoring report on human exposure to environmental chemicals (CDC, 2009) lists 153 chemicals measured in the blood and urine samples collected from NHANES participants. Some of the chemicals measured include: tobacco smoke markers, environmental phenols, sulfonylurea herbicides, organochlorine pesticides and metabolites, organophosphorus insecticides, metals and metalloids, perfluorinated compounds, volatile organic compounds (VOCs), polycyclic aromatic hydrocarbons, phthalates and others. In 2015, NHANES updated the data tables and produced a new report (CDC, 2015).

Findings from this program are used to determine the prevalence of major diseases and their risk factors. Information collected from a given survey could be compared with corresponding information collected in previous surveys. This allows scientists and health officials to detect any changes in the risk factors in the general population over time, and aids in identifying vulnerable populations, for example, seniors and children (Pirkle et al., 1994). Information collected on nutritional status is assessed in order to promote good health and disease prevention. Data from NHANES is also used in epidemiological studies and health sciences research. It is also used by other countries to compare or establish baseline exposure values for some environmental contaminants (Koch and Calafat, 2009). Results from NHANES help in establishing public health policies, beneficial health programs and robust services (http://www.cdc.gov/). 


\subsection{German Environmental Survey (GerES), Germany}

The German Environmental Survey (GerES) evaluates exposure of Germany's general population to environmental contaminants. It is conducted in collaboration with the German Health Survey for Children and Adolescents (KiGGS) and several Federal Environment agencies. The objectives of the GerES include the establishment of baseline measurements of environmental chemicals in the German population, derivation of health-based HBM-reference values to interpret HBM data, and helping to improve the understanding of the relationship between environmental exposure and health effects (http://www.umweltbundesamt.de/en).

The GerES I was conducted in 1985-1986. The sample size was 2,731 persons, 25-69 years of age, from 100 sampling locations in West Germany (http://www.umweltb undesamt.de/). The levels of chemicals in the air, tap water, dust around the homes, and the contents of vacuum cleaner bags were measured. Measurements were also made of chemicals in biological samples (including blood, urine, and scalp hair) provided by some of the participants. The whole blood samples were screened for cadmium, copper, lead, and mercury; morning urine samples were screened for arsenic, cadmium, chromium, copper, mercury, creatinine, pentachlorophenol (PCP), and cotinine; scalp hair was evaluated for aluminium, arsenic, barium, boron, cadmium, calcium, copper, iron, lead, magnesium, phosphorus, strontium, and zinc.

The second survey was conducted in 1990-1991 (GerES IIa) in West Germany. In this survey 2,524 adults aged 25 to 69 years and 453 children aged 6 to 14 years were included. Another survey, GerES IIb was conducted in 1991-1992 in East Germany. 1,763 adults, aged between 18-79 years and 359 children and adolescents aged between 6 to 17 years from 50 sampling locations participated in the survey. Like GerES I, chemicals were measured in several environmental media in the home environment (for example, water, dust, air) as well as in biological samples (including blood, urine, and scalp hair) provided by a-sub-sample of the participants. The biological samples were screened for chemicals outlined in the first survey, for adults as well as children (Seifert et al., 1999).

GerES III was performed in 1998. The sample size was 4822 adults, 18-69 years of age, collected from 120 sampling locations across Germany. Researchers measured the levels of chemicals (measured in the first survey) in the biological specimens from the participants. GerES IV is similar to GerES III but focused exclusively on children. It started in May 2003 and the field work was completed in May 2006, 1,800 children from 150 sampling locations across Germany participated in this survey (Schulz et al., 2007b). The GerES V survey, commenced in 2014 is scheduled to be completed in 2017. This survey, like GerES IV, focuses on children aged 3 to 17 years and is intended to assess the impact of chemical and noise pollutions on the health and wellbeing of the children. The scope of GerES is not only to analyse pollutants in blood and urine samples from participants, but also to include the identification of potential exposure pathways by which individual pollutants reach humans as well as to assess the changes in the environmental impact over several years. These German surveys will allow population health officials the opportunity to identify HBM concentrations of concern and initiate interventions to reduce exposures, internal doses and adverse health effects. 


\subsection{Flemish human biomonitoring program, Belgium}

In 2002, the Flemish government initiated a human biomonitoring program in Flanders, Belgium. It was conducted by the Flemish Centre of Expertise for Environment and Health, funded by the Flemish government (http://www.milieu-en-gezondheid.be). One of the main objectives of this program was to identify baseline values or reference values for environmental pollutants in order to establish the exposure of the Flemish population. The program has completed two cycles, Flemish Environment and Health Study I 2002-2006 (FLEHS I) and FLEHS II (2007-2011). More than 4,400 participants participated in FLEHS I. The biological samples (blood and/or urine) were collected from eight different regions of Flanders, for four age groups, newborn babies and their mothers, adolescents (14-15 years) and adults (50-65 years). The program measured a variety of biomarkers of exposure such as persistent chlorinated compounds, heavy metals, dioxins, and metabolites of PAHs (Schroijen et al., 2008).

FLEHS II (2007-2011), included three target age groups, newborns $(\mathrm{n}=250)$, 14-15 year old adolescents $(n=200)$ and 20-40 year old adults $(n=200)$. Participants living in all provinces in the Flemish region were included. A stratified sample design, with the sample size proportional to the size of the population in each province was used to make the study representative of the Flemish population. More than 40 biomarkers were analysed in blood, urine and hair samples collected from the participants (Schoeters et al., 2012). The results from these studies will allow characterisation of baseline xenobiotic concentrations and provide necessary data for developing reference values for environmental chemicals in the Flemish population.

\subsection{French Nutrition and Health Survey [Etude Nationale Nutrition Santé (ENNS)]}

The Etude Nationale Nutrition Santé (ENNS) is a nationally representative, cross-sectional biomonitoring survey in France carried out by Institut de Veille Sanitaire (InVS; French Institute for Public Health Surveillance) and Paris 13 University. The survey included adults and children, 3 to 74 years of age. The participants were randomly selected using a three-stage probability design based on the community, household and individual and stratified by geographical area (Fréry et al., 2012).

The general objectives of ENNS were to provide nationally representative data on diet, physical activity and nutritional status in the French population (http://www.invs.sante.fr/en). In addition this survey also provided background levels of several contaminants in the French general population. The participants were administered a questionnaire, followed by anthropometric and blood pressure measurement. They also provided biological samples (blood, urine and hair for adults and hair from children) in which several biomarkers of nutrition and exposure to environmental chemicals were measured. The data on diet were collected from the participants by means of three $24 \mathrm{~h}$ dietary recalls (Fréry et al., 2010, 2012). Exposure to chemicals in food and/or in the environment was measured by analysing 42 biomarkers that included metals (arsenic, cadmium, chromium, cobalt, lead, mercury, etc.), several pesticides (pyrethroids, organochlorines, organophosphorus) and non-dioxin-like PCBs (NDL-PCBs) (Fréry et al., 2010). These studies will assist health officials to explore links between nutrition, diet, environmental chemical exposures and health in the French population. 


\subsection{Consortium to perform human biomonitoring on European scale (COPHES/DEMOCOPHES), Europe}

Consortium to Perform Human Biomonitoring on European Scale (COPHES) was a partnership between academia, governmental agencies and industry in 17 European countries. Initiated in 2009, COPHES had 35 members with wide range of experience and expertise. COPHES was tasked to harmonise human biomonitoring methods so as to implement them in pan-European scale. The objective for a pan-European biomonitoring program was to collect information on exposure to environmental chemicals in the representative general population living in different countries in Europe. The methods developed in COPHES were tested in 17 countries in Europe (Joas et al., 2012). The pilot study termed as DEMOCOPHES (Demonstration of a study to Coordinate and perform human biomonitoring on a European scale) was initiated in 2011. The target population for DEMOCOPHES was children aged 6 to 11 years and their mothers aged up to 45 years. About 240 urine and hair samples (120 children and 120 mothers) were collected from each of the 17 participating countries. Qualified personnel visited the homes of the participants and administered a questionnaire (to gather supporting information on for example, personal characteristics, lifestyle, and home environment) and collected hair and morning urine samples. Cadmium, cotinine, and phthalate metabolites in urine and mercury in hair were measured in the samples collected from the participants. Qualified laboratories were chosen to perform these analyses who have participated in inter-laboratory comparison exercises as well as external quality assessment exercises (Schindler et al., 2014). The DEMOCOPHES study was concluded in 2012, demonstrating the possibility of a pan-European human biomonitoring program that will enable the comparison of biomonitoring results obtained from participating countries (http://www.eu-hbm.info/cophes). This successful demonstration of a pan-European HBM program demonstrates the opportunity to study public health impacts of lifestyle, diet, and environmental exposures on diverse communities.

\subsection{Korean National Health and Nutrition Examination Survey, Republic of Korea}

The Korean National Health and Nutrition Examination Survey (KNHANES) is a national health survey, initiated in 1998 by the Korea Institute for Health and Social Affairs and the Korea Health Industry Development Institute in the South Korea. The main goals of this survey are, "to assesses the health and nutritional status of Koreans, monitor trends in health risk factors and the prevalence of major chronic diseases, and provide data for the development and evaluation of health policies and programs in Korea" (https://knhanes.cdc.go.kr/knhanes/eng/sub01/sub01_02.do).

The KNHANES I (1998) and the two subsequent surveys in 2001 (KNHANES II) and 2005 (KNHANES III) were carried out triennially with an overall sample size of about 35,000 in each survey period. Since 2007, this survey was converted into an annual health survey with an approximate sample size of 10,000 people per year and carried out by the Korea Centers for Disease Control and Prevention.

KNHANES is a nationally representative cross-section survey with a multi-stage clustered probability design and includes non-institutionalised civilians aged one year or older. It consist of three components namely, health interview, health examination and nutrition survey. The health interview and health examination survey are carried out in a 
MEC by qualified medical professionals. The nutrition survey is carried out at the participants' households one week after the health interview. Information on several health indicators, demographic and socioeconomic variables are collected from the participants during the survey. Blood and urine samples is collected from study participants ten years and older during the health examination. Several biochemical indicators of diseases such as diabetes, dyslipidemia, hepatitis, and anemia, heavy metals such as mercury, lead and cadmium as well as tobacco smoke biomarker (cotinine) are measured in the biological samples (Kim, 2014; Kweon et al., 2014).

\section{Interpreting human biomonitoring data}

As described in Section 3, the availability of HBM data has increased substantially over the past decade, and consistent efforts have been made to use that information in population health risk assessment (Angerer et al., 2006, 2007; European Commission, 2009; Health Canada, 2012; Krewski et al., 2014). Such endeavours, within and across countries, have resulted in frameworks and general guidelines for the interpretation of HBM data in protecting human health (ECETOC, 2005; NRC, 2006). These guidelines will be summarised in the following sections.

\subsection{General considerations for interpretation of human biomonitoring data}

A series of factors should be explicitly considered in the evaluation of biomarkers in the context of population health risk assessment. These include the following:

- Analytical data quality: the analytical integrity of the biomonitoring data must be established before attempting any interpretation. Quality control and quality assurance (QA/QC) procedures must be comprehensive and encompass pre-analytical (e.g., sample collection and storage), analytical (analytical methods and protocols) and post-analytical (data analysis, data dissemination) phases. Procedures for sample collection, storage and transportation to the analytical laboratory must be standardised to protect sample integrity and avoid contamination. Similarly, the choice of the analytical method must be carefully considered with proper attention to the external contamination of the samples (Ye et al., 2013). Standard operating procedures for the analytical method should be developed and should be strictly followed during sample analyses (Padmanabhan et al., 2008; Schindler et al., 2014). Limits of detection and quantification must be established using standardised protocols. Robustness of the analytical method should be evaluated as a part of the analytical method validation. Other key parameters such as accuracy, linear dynamic range, and long-term precision must be carefully evaluated (Langlois et al., 2014; Magnusson and Örnemark, 2014). Analysis of samples must be carried out with fully validated analytical methods and supported by the use of quality control samples (Albertini et al., 2006; Calafat and Needham 2008; Smolders et al., 2009). Finally, the laboratory should participate in inter-laboratory exercise as and when available (Göen et al., 2012; Schindleret al., 2014; Vandenberg et al., 2014). 
- Choice of biomarkers: biomonitoring data on the most appropriate biomarker(s) in a relevant biological matrix are required to make meaningful conclusions concerning the exposure or potential population health effects. As PK factors determine the disposition of the chemical in the body, they play an important role in establishing sensitive and specific biomarkers to evaluate exposure in the HBM study. For example, diisononyl phthalate (DINP), one of the widely used plasticisers, is rapidly metabolised to mono-isononyl phthalate (MINP; a minor metabolite) which further undergoes oxidative reactions to form secondary metabolites (e.g., monohydroxy isononyl phthalate; MHINP) prior to excretion in urine (Koch and Angerer, 2007). Therefore, biomonitoring data on MHINP, as opposed to MINP, would provide a more accurate estimate of human exposure to DINP. In addition, PK information would also be useful to evaluate the metabolite's specificity. For example, phenol is one of the major metabolites of benzene. However, urinary phenol measurement is not a good indicator of benzene exposure as phenol also occurs naturally and can be found in other exposures such as cigarette smoke (McDonald et al., 2001; Arnold et al., 2013). Non-specificity could also arise from endogenous production of a biomarker in the body (e.g., ethylene oxide).

- Variability in biomarker concentration: the concentration of biomarkers in individuals based on convenience samples (e.g., spot urine samples) could vary considerably within a day and or between-days. The PKs of the biomarkers (e.g., elimination half-lives) as well as the exposure frequency plays a key role in determining temporal variability in biomarker concentrations (Aylward et al., 2012). In addition, the daily urine volume excreted, as well as the volume of each void, varies widely amongst individuals (Valentin, 2002). The volume of urine excreted by an individual depends on a range of factors including hydration status, fluid intake, seasonal variation, glomerular filtration rate, renal tubule function (secretion and resorption) and body size (Valentin, 2002). Generally children have a lower absolute urine excretion volume than adolescents and adults, based on body size (Valentin, 2002). These variations collectively referred to as 'urine dilution effects' could influence the concentration of biomarkers in the urine.

Ideally, measurement of a chemical or its metabolites in 24-h urine samples would be highly beneficial in HBM studies. However, collection of 24-h urine samples is cumbersome, placing a burden on study participants and, in the case of children, may even be impractical. Hence, large-scale population-based biomonitoring surveys such as NHANES and CHMS measure chemical contaminants in spot urine samples (random urine samples collected from study participants). Spot urine samples are also used in several epidemiological studies designed to characterise the relationship between exposure and health effects, for example in the MIREC cohort study described in the previous section and Center for the Health Assessment of Mothers and Children of Salinas (CHAMACOS), a cohort study which evaluated environmental chemical exposures and health impacts in women and their children (Eskenazi et al., 2003).

Population representative surveys usually employ large sample sizes to cover different age groups, ethnicity and gender in a given population. Therefore, population distribution of chemicals measured in such surveys inherently account for inter-and intra-individual variability. However, urine dilution effects become 
important when inferences are made on exposure levels based on biomonitoring data, for example between two sub-population groups (e.g., children vs. adults) or between individuals who falls in two different percentiles of a population distribution (Saravanabhavan et al., 2014; Hays et al., 2015). Thus, it is critical to adjust for urine dilution effects to avoid exposure misclassifications. As described in subsequent sections, this is particularly important for chemicals with short half-life.

- Evaluation of descriptive statistics: the biomarker data from large-scale general population biomonitoring surveys such as NHANES are typically skewed to the left (i.e., the majority of the people in the general population are exposed to low environmental levels). Therefore, using the geometric mean (GM) concentration to calculate daily intakes (DIs) provides a better estimate of the central tendency of the dose in the population than other measures. The 95 th percentile concentration is often used to derive the upper bound dose estimate. In general, individuals whose biomarker concentrations are higher than the 95th percentile population distribution are classified as highly exposed. However, half-life of the chemicals and the exposure frequency greatly influence the temporal variability of the biomarker concentrations in individuals. The urinary biomarker concentrations of chemicals with short half-life with respect to exposure frequency tend to exhibit higher temporal variability (Aylward et al., 2012). In such cases, high urinary concentrations in spot urine samples from individuals (who are typically classified as highly exposed) may not necessarily indicate continuous high exposure, but simply reflect 'peak' exposure which occurred shortly before the spot sample was obtained. Hence, using the 95th percentile concentrations for those measures in dose calculations would provide a conservative upper bound exposure estimate. Likewise, individuals whose biomarker concentrations fall at low percentiles (e.g., fifth percentile) of distribution for short-lived chemicals would indicate that the exposure occurred long before (at least a couple of half-lives) the urine sample collection.

- Analysis of pooled samples: while spot samples are typically taken in both national and smaller studies, pooled samples are also a cost-effective alternative (Heffernan et al., 2014). For example, Ye et al. (2012) examined concentrations of bisphenol A and several other phenols in pooled serum samples from 3 to 11 year olds in California. The AHW biomonitoring program included a comprehensive list of environmental contaminants measured in over 1,300 children and 28,000 pregnant women. Samples were pooled within each age class, with three replicate pools for each age class (AHW, 2008). In addition, for certain analyses (e.g., dioxins) the available analytical methods demand large volumes of blood (Rawn et al., 2012). It is often not possible (and may be considered as unethical) to collect large volumes of blood, particularly from infants and children. In such situations, analysis of chemicals in pooled blood samples from several participants (say for example based on age groups) would be very practical and convenient. Careful considerations must be given to how samples are pooled (number of specimen per pool and the subpopulation represented) as well as interpretation of HBM data from pooled samples (Schisterman et al., 2005; Verret et al., 2010). This is particularly important when ascertaining infrequent or peak exposures of interest as pooled samples only provide an estimate of central tendency. 
It is highly desirable to relate biomonitoring data to health effects directly; however, at the present time there are only a few chemicals, such as blood lead and methyl mercury, for which sufficient epidemiological evidence exists to establish a direct relationship between biomarker concentration and health effects (Trasande et al., 2005; Clewell et al., 2008; Health Canada, 2013a). For most of the environmental chemicals, PK and toxicity data from animal experiments and exposure information in humans in conjunction with valid HBM data (in terms of analytical quality, biomarker selection and its stability in the biological matrix, and the relationship between the biomarker to the chemical exposure) are required to relate chemical exposure to health effects (Albertini et al., 2006; NRC, 2006). However, estimates of population health risks can be estimated based on animal toxicity studies, and certainly the HBM concentrations can be compared across time and between different populations, supporting public health interventions (Krewski et al., 2014).

\subsection{Health-based exposure guidance values}

Health-based exposure guidance values for several chemicals have been derived by different regulatory agencies, and can be identified in authoritative national and international guidance documents. These include RfDs (reference dose) and RfCs (reference concentration) from the US Environmental Protection Agency (USEPA, 2015); minimal risk levels (MRLs) from the US Agency for Toxic Substances and Disease Registry (ATSDR, 2015); acceptable daily intake (ADIs) from the World Health Organization(WHO) (Lu, 1988); and ADI/ tolerable daily intake (TDI)/RfD from Health Canada (Health Canada, 1995, 1996). These guidance values refer to different routes of exposure (oral, inhalation, dermal), different exposure durations (chronic, sub-chronic and acute) and also different biological responses (e.g., cancer and non-cancer, including reproductive and developmental effects). Several tools and guidelines have been developed for calculating guidance values (see http://www.epa.gov/risk/guidance.htm). It should be noted that the guidance values developed by different regulatory agencies can differ from one another. This might be due to the choice of the critical effect, key study used to assess the point of departure (POD) and/or uncertainty factors (UF) applied. As an example, different exposure reference values have been developed for triclosan: $300 \mu \mathrm{g} / \mathrm{kg} /$ day [no observed adverse effect level (NOAEL)/UF] (USEPA, 2008), $120 \mu \mathrm{g} / \mathrm{kg} / \mathrm{day}$ (NOAEL)/UF) (European Commission, 2009; Krishnan et al., 2010b) and ADI: $80 \mu \mathrm{g} / \mathrm{kg}$ /day (NOAEL/composite adjustment factors) (Health Canada, 2012). For many chemicals health-based guidance values developed by government agencies using transparent, peer-reviewed methods are unavailable. In such cases, if sufficient animal toxicity data are available, provisional (screening level) guidance values can be derived using the POD values from the key study, after applying adjustment factors (Sand et al., 2011; Becker et al., 2012). Based on the robustness of the toxicity data (e.g., whether the critical data are based on chronic versus acute animal studies), additional UF may need to be considered. For several high production volume chemicals, the lowest observable adverse effect level (LOAEL) and NOAEL values could be used as the starting point to derive screening level health-based exposure guidance values (Becker et al., 2012). Chemicals that lack robust toxicity data pose a major challenge for deriving health-based guidance values. In such cases, the threshold of toxicological concern (TTC) method may be used to determine provisional tolerable dose levels from historic data and a distribution of NOAEL values (Kroes et al., 2005; Becker et al., 2012). 


\subsection{PK data}

Some understanding of the PK characteristics of the chemical is essential to establish a relationship between the external exposure dose and the HBM concentrations in biological specimens. For some chemicals, dosing experiments conducted in human volunteers have been used to develop such relationships (Bartoníček, 1962; Huang et al., 1994; Ukai et al., 1994; Koch and Angerer, 2007). Most of these studies were conducted in occupational settings and primarily focused on inhalation exposures to VOCs. As only a few exposure concentrations were included in those studies, the shape of the curve describing the relationship between the external dose and the biomarker concentration is not well defined. In such cases, a linear relationship between the biomarker concentration and the exposure dose is generally assumed. In some human studies, PK data are derived from exposure to a single oral dose (Koch and Angerer, 2007). In such cases, if the rate of absorption is assumed to be rapid compared to the rate of elimination, the exposure dose can be related to steady-state biomarker concentrations in blood or urine using simple first-order kinetics (Sandborgh-Englund et al., 2006; Hays et al., 2007).

In some instances, PK data collected in animal toxicity studies are used directly as surrogates for human PK characteristics. For the safety evaluation of triclosan from consumer products, the European Commission used the NOAEL of $12 \mathrm{mg} / \mathrm{kg} \mathrm{bw} / \mathrm{d}$ for hematological effects from a rat chronic study (European Commission, 2009). The margin of safety (MOS) was estimated by comparing the expected triclosan exposure dose (using exposure modelling) in humans to that of the NOAEL from the critical rat study.

More often UF are applied when a NOAEL or LOAEL from animal studies is extrapolated to human exposures. UF are applied based on several considerations including the availability of biomarker concentration data at the critical dose, mode of action (MOA) of the chemical, and an understanding of the similarities (and differences) between the metabolic pathways of the chemicals in animals and humans. Even if PK data for the critical toxicity study are not available for the derivation of the guidance values, parameters from related animal experiments can be used with additional extrapolations using allometric scaling factors (Kirman et al., 2003).

\section{HBM data interpretation approaches}

Forward and reverse dosimetries are two major approaches that are currently utilised for interpretation of HBM data in health risk assessments. In Figure 1, both these approaches are illustrated in the context of an exposure-health effects continuum (exposure $\rightarrow$ internal dose $\rightarrow$ health effects). As mentioned earlier, the chemical-specific biomarker concentrations could be directly related to the internal dose of a given chemical. The forward dosimetry approach utilises human/animal PK data and/or PK modelling to estimate reference biomarker concentrations in relevant biological matrices from existing human guidance values (e.g., TDI, RfD, RfC etc.) or POD values from animal experiments (Hays et al., 2007, 2008a; Clewell et al., 2008; Angerer et al., 2011). The HBM data are compared against these reference biomarker concentrations to evaluate health risks and set priorities for risk assessment (Aylward et al., 2013) (i.e., relating internal dose to health effects; Figure 1). Reverse dosimetry on the other hand, aims to 
estimate the exposure dose that would result in the measured biomarker concentration (i.e., connecting internal dose to exposure; Figure 1).

\section{Forward dosimetry-based methods}

\subsection{Biomarker guidance values in occupational settings}

The forward dosimetric approach has been used in occupational settings to derive biomarker guidance values for over 20 years (Lowry, 1986; ACGIH, 2015). These include threshold limit values (TLVs) and the biological exposure indices (BEIs) developed by the American Conference of Governmental Industrial Hygienists (ACGIH), biological tolerance values (BATs) and biological guidance values (BLW) developed by the German Commission for the Investigation of Health Hazards of Chemical Compounds in the Work Area, (MAK Commission) (Angerer et al., 2011). These values are based on workers' exposure to chemical and physical agents under specific exposure scenarios in occupational settings. For example, the ACGHI committee which formulates BEI and TLVs consists of experts in occupational hygiene and public health. In deriving these guidance values, the committee carries out a critical review of pertinent scientific literature to identify NOAEL or LOAEL of the chemical from relevant animal or human exposure studies. Emphasis is also placed on dose/concentrations at which the critical effects appear to be reversible (ACGIH, 2015). These guidance values help trained occupational hygienists make decisions about the safe levels of chemical exposure in the occupational environment. However, neither TLV nor BEI represents a bright line between safe and unsafe exposure levels (ACGIH, 2015).

\subsection{Biomonitoring equivalents (BE)}

A biomonitoring equivalent or $\mathrm{BE}$ is defined as "the concentration or range of concentration of chemicals or their metabolites in a biological medium (blood, urine, or other medium) that are consistent with an existing health-based exposure guidance value such as reference dose (RfD) or tolerable daily intake or with a cancer-based exposure guidance value such as a risk-specific dose (e.g., the dose associated with a $1 \times 10^{-4}$ cancer risk)" (Hays et al., 2007, 2008a). BEs are screening tools which are useful for interpreting population level HBM data in the population health risk context, and are conceptually similar to BEIs established for occupational settings.

Figure 2 depicts general approaches to derive BE values based on the availability of animal PK and toxicity data. For compounds that have sufficient PK data, the existing exposure guidance values such as TDI or RfDs can be directly converted into corresponding biomarker concentrations (Figure 2; pathway 1). For chemicals without exposure guidance values, BE values can be derived from the POD values from animal toxicity studies. These POD values are first converted into human equivalent POD values by applying appropriate inter-species UF (Figure 2; pathway 2). Then by using the PK data or PK models, the human equivalent POD values are converted into $B E_{P O D}$ values. Finally, the $B E_{P O D}$ value is converted into a $\mathrm{BE}$ value by applying appropriate UF (Figure 2; pathway 2). 
Figure 2 Approaches for the derivation of BE values for compounds with varying guidance and pharmacokinetic data availability

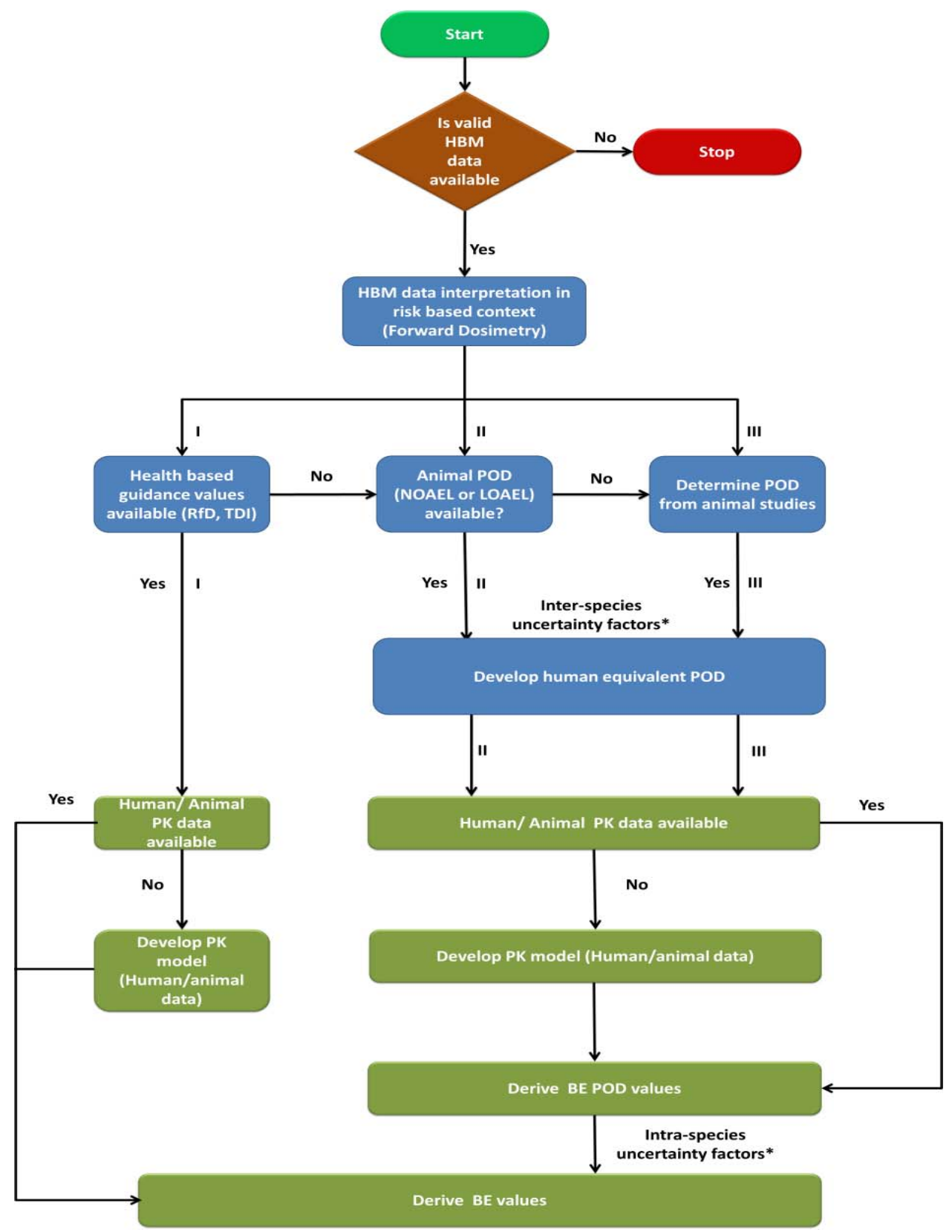

Notes: HBM: human biomonitoring data; RfD: reference dose; TDI: tolerable daily intake; POD: point of departure; NOAEL: no observed adverse effect level; LOAEL: low observed adverse effect level; PK data: pharmacokinetic data; uncertainty factors*: inter- and intra-species factors are based on pharmacokinetic information; BE POD: biomonitoring equivalent at human equivalent POD; $\mathrm{BE}$ - biomonitoring equivalent 
Table 2 List of derived BE values along with risk assessment exposure guidance values for selected environmental chemicals

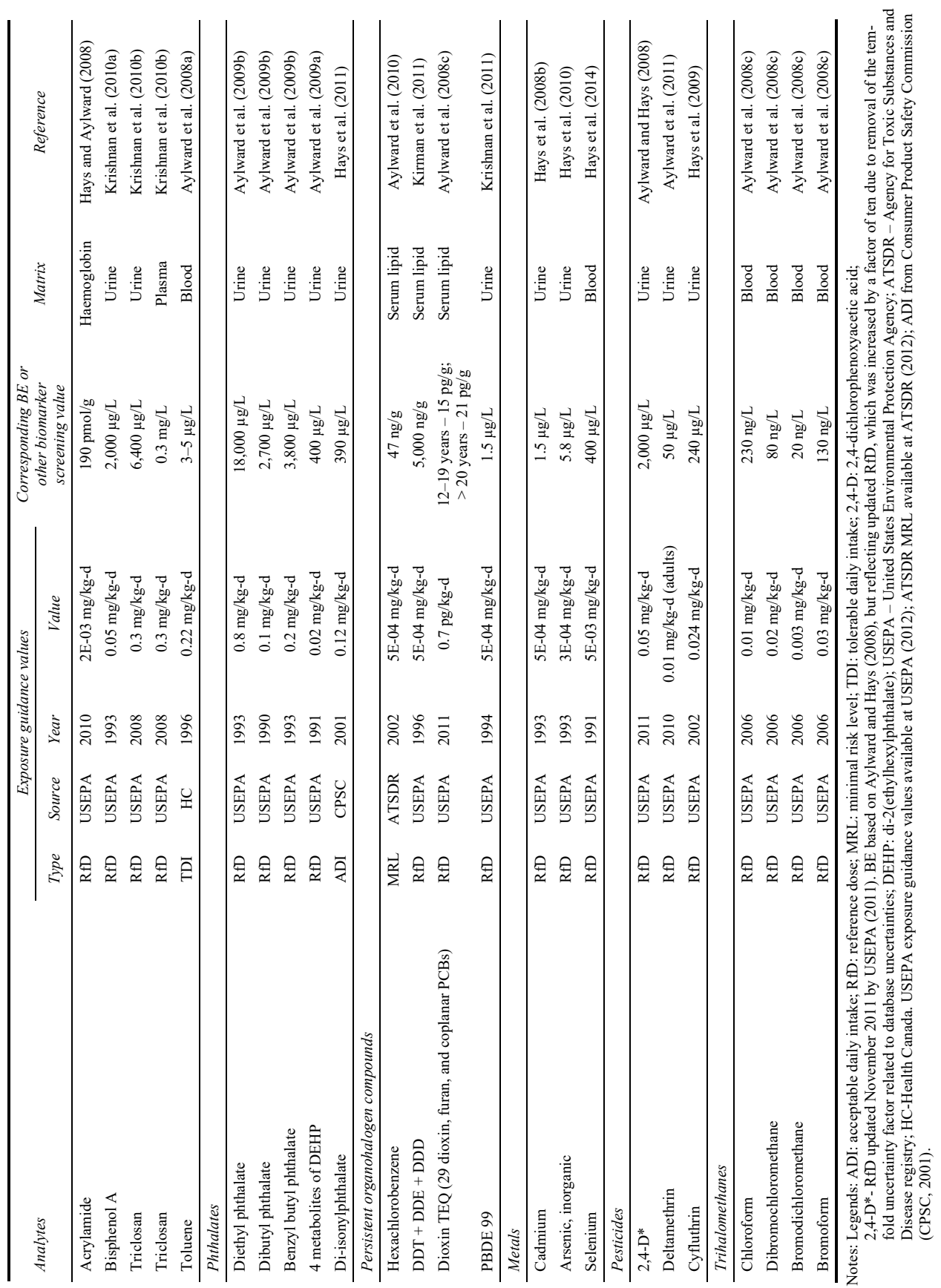


BE derivation makes a variety of assumptions pertaining the relevance and applicability of internal dose of chemicals in predicting health effects (Hays et al., 2007, 2008a). The primary assumption is that the internal dose of a chemical is a better predictor of health effects than the external exposure dose and the measured biomarker(s) concentrations in biological matrices are relevant in predicting chemical toxicity (e.g., lead and methyl mercury). Secondly, it assumes that the exposure guidance values are reasonable and health-protective of all subpopulations. Thirdly, BEs are derived based on steady-state conditions (i.e., intake and elimination rates of the chemicals are equal). In addition, BEs inherits all the assumptions on which the exposure guidance and/or POD values are based.

The choice of biomarker for BE derivation takes into account the MOA of the chemical and its toxicokinetics, among other factors [see for example BE derivation for toluene in Aylward et al. (2008a)]. However, it is not always possible to choose the biomarker that is most closely related to the MOA and/or the MOA is not known. Exposure guidance values that are specific to an exposure event such as chronic, intermediate or acute duration exposures could also be used as the basis for the derivation of BEs, provided such BE values are applied to HBM data that has been generated under the same specific exposure conditions as the guidance values (Hays et al., 2007, 2008a). $\mathrm{BE}$ values for several environmental chemicals in urine as well as blood have been derived (Table 2).

\subsubsection{Deriving BEs for biomarkers in blood}

Figure 3 depicts approaches that could be used to derive BEs using blood-based biomarker concentrations depending on the nature and the availability of PK data. In a simplest case, if the PK relationship between the blood biomarker concentration and the external dose is available in humans, but not known in animal models, then such a relationship could be used to extrapolate the guidance values to corresponding biomarker concentrations (steady-state approach; Figure 3). The BEs for several VOCs as well as 2, 4-dichlorophenoxyacetic acid has been derived using this approach (Aylward and Hays, 2008; Aylward et al., 2010).

Currently, PK data for many chemicals are available in animals which can be effectively used for deriving BEs for blood-based biomarkers using the internal dose extrapolation approach (Figure 3). This approach requires some understanding of the MOA (critical tissues) and dosimetry (the concentration in the critical tissue). This approach has been used to derive BE for hexachlorobenzene (HCB), acrylamide and trihalomethanes (Aylward et al., 2008b, 2010; Hays and Aylward, 2008). When there is sufficient evidence that the blood biomarker concentration is very closely related to the tissue concentration (from animal or human studies), the internal dose approach presented in Figure 3 could be simplified by assuming blood biomarker concentration as a surrogate for tissue concentration in animals. After applying appropriate inter-species UF the blood biomarker concentration in animals could be converted to equivalent blood biomarker concentration in humans $\left(B E_{P O D}\right)$. Krishnan and co-workers (Krishnan et al., 2010b) used this approach to derive the BE for plasma triclosan. 
Figure 3 Schematic representation for deriving blood-based BE values

\section{Steady-state approach}

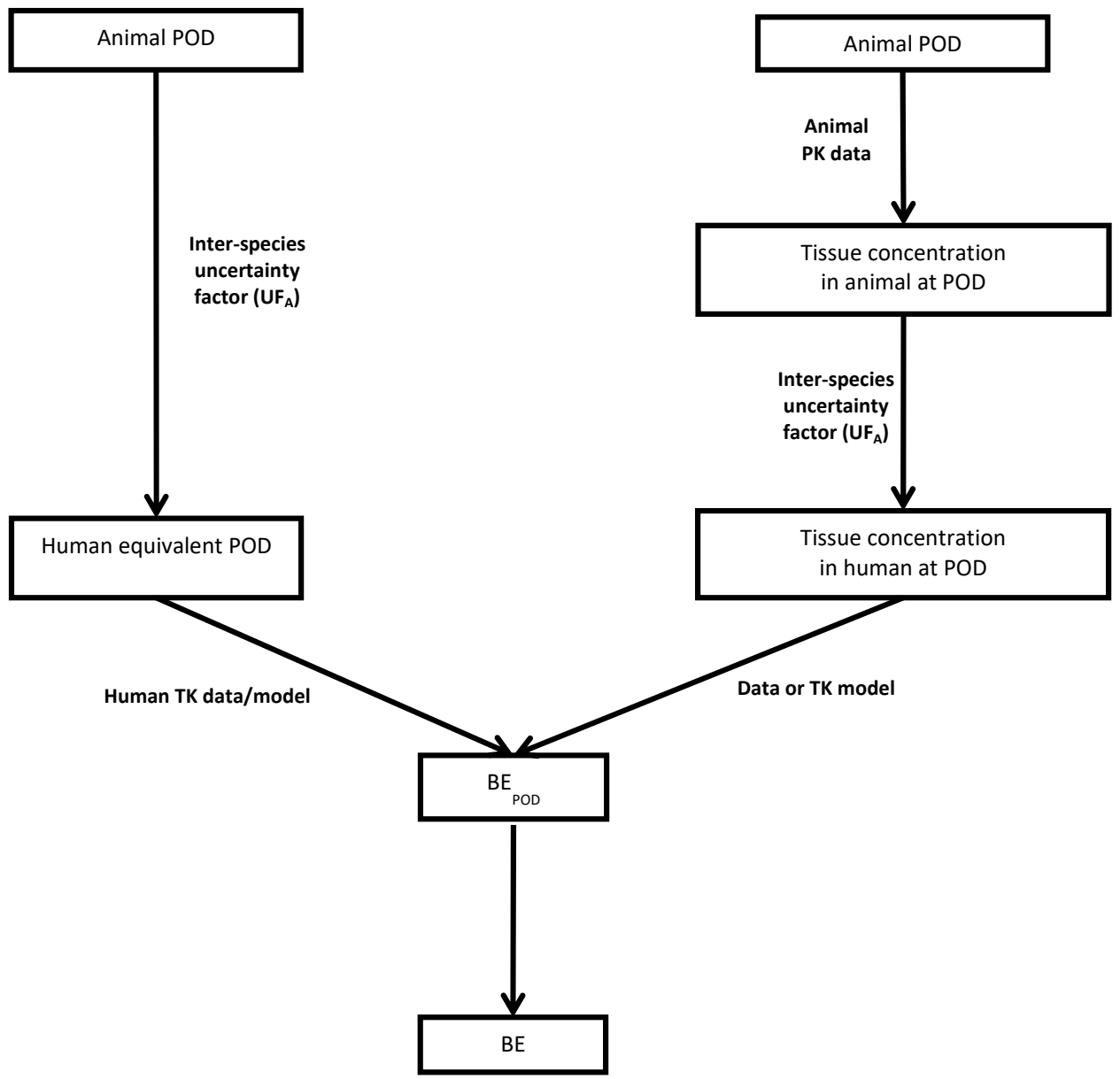

Notes: POD: point of departure; TK data: toxicokinetic data; uncertainty factors*: inter- and intra-species factors are based on pharmacokinetic information

Source: Figure modified from Angerer et al. (2011)

\subsubsection{Urinary biomarker concentration}

For chemicals that are primarily excreted in urine, either as a parent compound or as metabolites, a urinary mass balance approach, similar to the steady-state approach depicted in Figure 3, can be used to derive BEs (Angerer et al., 2011). In this approach, first the human equivalent POD is determined by dividing the animal POD using interspecies UF. At steady-state the amount of chemical excreted in urine would be equal to the amount ingested multiplied by the fractional urinary excretion (FUE). The FUE is a 
molar ratio of the amount of chemical appearing in urine to that of the administered dose (Koch et al., 2003). In most cases, the FUE is determined using actual excretion data from controlled human dosing experiments. The measured FUE generally varies among individuals [e.g., triclosan; (Sandborgh-Englund et al., 2006)]. However a central tendency measure of FUE is chosen in BE estimation as the variability introduced due to differences in the FUE values is usually smaller than the inter-individual variability in the population (Krishnan et al., 2010b).

Chemical mass balance established under steady-state conditions is used to convert the human equivalent POD ( $\mu \mathrm{g} / \mathrm{kg}$ bw/day) into corresponding urinary concentration $\left(B E_{P O D} ; \mathrm{ug} / \mathrm{L}\right)$ using the following equations.

- $\quad$ Based on urine volume

$$
B E_{P O D}(\mu g / L)=\frac{[D(\mu g / k g / d a y) \times B W(k g) \times F U E]}{V_{24 h}(L / \text { day })}
$$

- $\quad$ Based on urinary creatinine

$$
B E_{P O D}(\mu g / g C r)=\frac{[D(\mu g / k g / d a y) \times B W(k g) \times F U E]}{C r_{24 h}(g / \text { day })}
$$

where $B E_{P O D}(\mu g / L)$ and $B E_{P O D}(\mu g / g \quad C r)=B E_{P O D}$ based on volumetric or creatinine-adjusted urinary concentrations; $D=$ external dose (human equivalent POD in

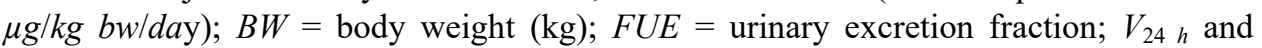
$\mathrm{Cr}_{24}{ }_{h}=$ estimated average 24-h urinary volume and creatinine excretion rate (CER), respectively.

The only PK data requirement for this method is the FUE in humans. FUE values for some chemicals are tabulated in Table 3. Some of the chemicals for which this approach has been used to derive urinary BE values include triclosan (Krishnan et al., 2010b), bisphenol A (Krishnan et al., 2010a), several phthalates (Aylward et al., 2009b), and inorganic arsenic (Hays et al., 2010).

When multiple urinary metabolites are detected in humans, the BE derivation approach takes into account the sum of all metabolite concentration. For example, metabolism of di(2-ethylhexyl)phthalate (DEHP) leads to the formation of primary metabolite mono (2-ethylhexyl)phthalate (MEHP) and several secondary metabolites such as mono (2-ethyl-5-hydroxyhexyl)-phthalate (MEHHP), mono(2-ethyl-5-oxohexyl)phthalate (MEOHP), mono(2-ethyl-5-carboxypentyl)-phthalate (MECPP), and mono [(2-carboxymethyl)hexyl] phthalate (MCMHP) (Koch et al., 2005; Silva et al., 2006). Therefore, urinary BE for DEHP were derived using the sum of three metabolites (MEHP, MEHHP, MEOHP), four metabolites (MEHP, MEHHP, MEOHP, and MECPP), and five metabolites (MEHP, MEHHP, MEOHP, MECPP, and MCMHP) (Aylward et al., 2009a). 
Table 3 FUE of different chemicals in humans, compiled from different studies

\begin{tabular}{|c|c|c|c|}
\hline Chemical & Urinary biomarker & Mean FUE & Reference \\
\hline Deltamethrin & DBCA & 0.15 & Aylward et al. (2011) \\
\hline \multirow[t]{3}{*}{ Inorganic arsenic } & As-III + As-V & 0.12 & Hays et al. (2010) \\
\hline & MMA & 0.15 & \\
\hline & DMA & 0.34 & \\
\hline Cyfluthrin & FPBA & 0.25 & Leng et al. (1997) \\
\hline Acrylamide & $\begin{array}{l}\text { Acrylamide mercapturic } \\
\text { acid }\end{array}$ & 0.5 & $\begin{array}{l}\text { Boettcher et al. (2006) and } \\
\text { Fuhr et al. (2006) }\end{array}$ \\
\hline Triclosan & Triclosan & 0.54 & $\begin{array}{l}\text { Sandborgh-Englund et al. } \\
\text { (2006), reviewed in Health } \\
\text { Canada 2012) }\end{array}$ \\
\hline Dibutyl phthalate & MBP & 0.55 & Anderson et al. (2001) \\
\hline Diethyl phthalate & MEP & 0.6 & $\begin{array}{l}\text { Saravanabhavan et al. } \\
\text { (2014) and Calafat and } \\
\text { McKee (2006) }\end{array}$ \\
\hline Benzylbutyl phthalate & $\mathrm{MBzP}$ & 0.6 & Anderson et al. (2001) \\
\hline \multirow{4}{*}{$\begin{array}{l}\text { Di (2-ethylhexyl) } \\
\text { phthalate }\end{array}$} & МEHP & 0.05 & Koch et al. (2005) \\
\hline & МЕHНP & 0.19 & \\
\hline & MEOHP & 0.11 & \\
\hline & МЕСРP & 0.18 & \\
\hline \multirow[t]{4}{*}{ Di-isononyl phthalate } & MiNP & 0.02 & Koch and Angerer (2007) \\
\hline & OH-MiNP & 0.2 & \\
\hline & Oxo-MiNP & 0.11 & \\
\hline & Carboxy MiNP & 0.11 & \\
\hline $\begin{array}{l}\text { 2,4-dichlorophenoxyacetic } \\
\text { acid }(2,4-D)\end{array}$ & $\begin{array}{l}\text { 2,4-dichlorophenoxyacetic } \\
\text { acid }\end{array}$ & 1 & Aylward and Hay (2008) \\
\hline Bisphenol A & Bisphenol A & 1 & Krishnan et al. (2010a) \\
\hline
\end{tabular}

Notes: DBCA: 3-(2,2-dibromovinyl)-2,2-dimethylcyclopropane carboxylic acid; As-III: Arsenic(III)acid; As-V: Arsenic(V)acid; MMA: monomethyl arsenic; DMA: dimethyl arsenic; FPBA: 4-fluoro-3-phenoxybenzoic acid;

MEP: monoethyl-phthalate; MBP:monoiso-butyl-phthalate;

MBzP: monobenzyl-phthalate; MEHP: mono(2-ethylhexyl)-phthalate;

MEHHP; mono(2-ethyl-5-hydroxyhexyl)-phthalate;

MEOHP: mono(2-ethyl-5-oxohexyl)-phthalate;

MECPP: mono (2-ethyl-5-carboxypentyl)-phthalate;

MiNP: monoisononyl-phthalate; OH-MiNP: mono-(hydroxy-iso-nonyl)-phthalate;

Oxo-MiNP: mono (oxo-isononyl)-phthalate;

Carboxy MiNP: mono(carboxy-iso-octyl)-phthalate;

2,4-D: 2,4-Dichlorophenoxyacetic acid.

Source: Table modified from Angerer et al (2011) 


\subsubsection{Strengths and limitations of BE approach}

Unique BE values are derived for each chemical taking into consideration the PK data and models, MOA and the toxicity of the chemical as well as the available exposure guidance values. BE values could be used to screen HBM datasets from general population studies or from targeted cohort studies in order to identify chemicals that are below, equal to/near, or above the existing exposure guidance values. Therefore, BEs serve as useful screening tools to assess HBM data in the human health risk context, providing an approach for ranking exposures and potential risks (Hays et al., 2007, 2008a). Moreover, the BE concept is easy to communicate and explain to the general public (Lakind et al., 2008).

On the other hand, BE values cannot be used either to interpret HBM data for individuals or as a diagnostic tool to assess 'safe' or 'unsafe' levels of exposure to chemicals. Exceedance of $\mathrm{BE}$ value in a single sample (or time point) does not necessarily mean continuous elevated exposure condition exists and would not trigger risk management initiatives. Comparison of HBM data with BE does not allow a direct population health impact assessment, it does however provide an approach for prioritising exposures and populations at potential risk. The BE approach does not capture temporal variability associated with compounds with short half-lives. Moreover, it assumes that the FUE is constant irrespective of the route of exposure.

\subsubsection{Uses of BEs in risk characterisation}

In traditional screening-level human health risk assessment, characterisation of health risk due to exposure to a chemical is often carried out by comparing exposure level prevalent in a given population or scenario to a reference dose (e.g., RfD, RfC). The hazard quotient (HQ), often calculated for a given exposure pathway, is a simple ratio of exposure dose $(\mu \mathrm{g} / \mathrm{kg}$ bw/d) or concentration to a reference dose $(\mu \mathrm{g} / \mathrm{kg} \mathrm{bw} / \mathrm{d})$ or concentration used to assess health risk for non-carcinogenic endpoints (USEPA, 2005). As described in the previous section, the BE for a given chemical is a translation of reference dose to internal dose using relevant PK data. This allows for the direct comparison of the biomarker concentration in a relevant biological matrix (e.g., urine) to $\mathrm{BE}$ (derived using appropriate PK data for urine) for a given chemical similar to the HQ approach used in traditional risk assessment. The following equation can be used to calculate the HQ for non-cancer end points (Aylward et al., 2013)

$$
\mathrm{HQ}=\frac{[\text { Biomarker }]}{\mathrm{BE}}
$$

Similar to traditional risk assessment, chemicals for which the HBM data results in a HQ $<<<1$ suggest a low priority for risk assessment. Chemicals whose HQ values are near 1 or above 1 indicate that the exposure levels are near or above the benchmark dose underlying that $\mathrm{BE}$ value and hence warrant further investigation and risk-assessment follow up.

In a similar fashion, MOS could be calculated using the following equation

$$
\operatorname{MOS}=\frac{\mathrm{BE}}{[\text { Biomarker }]}
$$


It should be noted that the $\mathrm{BE}$ approach inherently captures exposures via all exposure routes and pathways as it uses internal dosimetry to estimate risk.

While dealing with carcinogenic chemicals, the traditional risk assessment estimates cancer risk using unit risk factor or cancer slope factor $\left(\mathrm{q}^{*}\right)$. The risk specific dose (RsD) for a given chemical could be derived at an acceptable risk level (usually $1 \times 10^{-6}$ ). BEs (i.e., steady-state blood concentration) corresponding to the RsD could be derived using a suitable $\mathrm{PK}$ or PBPK model. The $\mathrm{BE}_{\mathrm{RsD}}$ for several chemicals, specifically for many VOCs, have been derived (Aylward et al., 2010).

\subsection{Human biomonitoring values (HBM-I and HBM-II)}

The German Human Biomonitoring Commission of the Federal Environment Agency advises the agency on all HBM issues. As part of its functions, the Commission has established two health-based biomonitoring guidance values, known as HBM-I, and HBM-II values, based on sound epidemiological and toxicological studies (HBM Commission, 2007, 2014). The HBM-I value is defined as "the concentration of a substance in human biological material below which - according to the knowledge and judgment of the commission and with regard to the substance under consideration - there is no risk for adverse health effects, and consequently, no need for an action" (HBM Commission, 2007). The HBM-I value is an alert level and the exceedance of HBM-I for a measure in an individual would prompt further toxicant measurements in his/her blood and urine samples. Other persons living in the same environment may also be included for further analyses (Ewers et al., 1999). The HBM-II values are intervention levels and are defined as, "the concentration of a substance in human biological material above which - according to the knowledge and judgement of the commission and with regard to the substance under consideration - there is an increased risk for adverse health effects, and consequently, an urgent need to reduce exposure and provide individual biomedical care" (HBM Commission, 2007). When HBM-II levels are exceeded for any measure in an individual, further toxicant measurement in the biological specimen are performed immediately. Moreover, actions are taken to reduce the individual's exposure and the person is referred to a medical examination for assessing possible health effects (Ewers et al., 1999).

Prior to 2007, HBM-I values were derived solely based on the results from epidemiological studies and hence were applicable at an individual level. HBM-I values for chemicals such as cadmium, mercury, pentachlorophenol, and thallium in urine or blood (Schulz et al., 2011) and PCBs in blood were developed using this approach. Since epidemiological data for many chemicals are not readily available the German HBM Commission has adopted a new approach in 2007 and again in 2014 to derive HBM-I values based on tolerable exposure values or key animal studies and the respective POD. Therefore, both BEs and most HBM-I values for chemicals are based on similar assumptions, data requirements and approaches (Angerer et al., 2011). Currently, HBM-I values are available for cadmium (in urine), mercury (in blood and urine), thallium (in urine), pentachlorophenol (in serum and urine), and PCBs (in blood) that were based on epidemiological studies. The HBM-I values for DEHP metabolites (in urine), DINCH metabolites (in urine), and bisphenol A (in urine) were developed using exposure guidance values. Further HBM-I values for glycol ethers metabolised to methoxy acetic acid (MAA) (in urine), DPHP metabolites (in urine), HBCDD (in lipid), N-ethyl-2pyrrolidon (NEP) metabolites (in urine), 2-mercaptobenzothiazole (2-MBT) (in urine) 
and triclosan (in urine) were derived based on a sound POD of an animal study. The HBM-II values are available for cadmium (in urine), mercury (in blood and urine), and pentachlorophenol (in serum and urine) (Angerer et al., 2011) as well as for PCBs in blood and NEP metabolites in urine (http://www.umweltbundesamt.de).

\subsection{Summary for forward approach}

Forward dosimetric approaches attempt to interpret HBM data by converting exposure guidance values into corresponding internal doses (biomarker concentrations). For this purpose, reference biomarker concentrations such BEIs, BEs and HBM-I values have been derived for many chemicals. These reference biomarker concentration values are simple to derive and are based on the available data on toxicity/exposure guidance values. Therefore, forward dosimetry approaches are useful screening tools to evaluate and prioritise HBM data in a health-risk-based context. However, these approaches neither provide quantification of exposure doses nor help to evaluate the source and pathways of chemical exposure.

\section{Reverse dosimetry-based methods}

Reverse dosimetry methods utilise human PK data as well as PK modelling to convert HBM concentrations into exposure doses (e.g., daily intakes) and/or possible exposure scenarios that are consistent with the biomonitoring data (Clewell et al., 2008; Georgopoulos et al., 2009). For chemicals that have sufficiently known sources and exposure pathways, more elaborate dose-reconstruction scenarios can be formulated using probabilistic models (Lyons et al., 2008; Georgopoulos et al., 2009; Tan et al., 2012).

\subsection{Daily intake (DI) estimations}

This is one of the simplest methods in reverse dosimetry. DI estimations using biomonitoring data are carried out with simple non-compartmental or compartmental (one or two compartment) PK models (David, 2000; Kohn et al., 2000). This approach may over-simplify the PK process in the biological system and assumes continuous exposure with steady-state body concentrations and cannot account for episodic exposures that often occur in real life scenarios (Lakind and Naiman, 2008; Lakind et al., 2011). Nevertheless, owing to its simplicity, the DI calculation serves as a first approximation to the intake dose of chemicals which can be derived using minimal PK data.

\subsubsection{Estimation of DI using urinary biomarker concentrations}

A generic expression for estimating DI of a chemical using urinary biomarker data is given below:

$$
\text { Daily intake }=\frac{\text { Urinary concentration } \times \text { Adjustment factor } \times M_{p}}{\text { Body weight } \times F U E}
$$


To minimise the effect of urine dilution, the urinary biomarker concentration data from spot urine analyses are adjusted using one of the following methods (Mage et al., 2004, 2008; Lakind and Naiman, 2008; Lakind et al., 2011; Huber et al., 2011). Based on the method employed, the urinary concentration and the adjustment factor terms in the above equation would change while all other terms remain the same. $M_{p}$ refers to molecular weight of the parent compound and FUE refers to FUE factor (see Section 5.3.2.). Specific DI equations pertaining to each adjustment method are provided in Table 4.

- Urine volume-adjustment: in this approach, the concentration of the biomarker in spot urine samples $\left(C_{\text {Volumetric }} ; \mu\right.$ mole/ $\left./ \mathrm{L}\right)$ is assumed to be representative of the concentration over a 24-hour time period. The total urine output (adjustment factor) for the individual is estimated either based on their urine flow rate (with the added assumption that the urine flow rate is constant) or based on the default reference values published in epidemiological studies (e.g., Valentin, 2002). Although this method does not account for variability in excretion rates of the chemical and/or metabolites, the differences in the concentration of the chemical excreted are expected to be randomly distributed and hence at a population level, the DI calculation would provide a representative estimate (Lakind and Naiman, 2008).

- Creatinine adjustment approach: creatinine is a by-product of muscle metabolism and in a given individual creatinine is excreted in urine at a more or less constant rate, as a function of renal blood flow and glomerular filtration rate. Hence, creatinine serves as an endogenous biomarker which can be used to account for urinary dilution (Boeniger et al., 1993). In this method, the creatinine adjusted urinary biomarker concentration $\left(C_{C r \text {-adjusted }} ; \mu\right.$ moles $\left./ \mathrm{g} \mathrm{Cr}\right)$ together with the CER (adjustment factor) is used to estimate the DI (Table 4). Muscle mass, and hence the amount of creatinine excreted by humans, depends on several factors such as age, gender, body weight, and diet (Mage et al., 2004, 2008; Barr et al., 2005). Recently, Mage et al. (2008) modelled CER in humans (> six years). These equations can be used to estimate CER in the general population. However, it should be noted that the CER is highly variable in infants and neonates, and hence this approach is not recommended for the DI estimation in these population sub-groups (Remer et al., 2002; Mage et al., 2008). It has also been noted to be highly variable in children. Kissel et al. (2005) found as much as 70-fold inter-individual variation in creatinine concentrations among children aged two to five years (across all samples) and a 16-fold intra-individual variation (within a single 24-h period). Moreover, this method assumes that the measured urinary biomarker behaves similarly to creatinine in the kidneys, i.e., the chemical is cleared predominantly by glomerular filtration and is not secreted or reabsorbed by the renal tubules. Therefore, this method is appropriate only in cases where the chemical/metabolite is excreted by the kidneys by glomerular filtration, like creatinine (Boeniger et al., 1993). Otherwise, the creatinine correction approach would introduce a bias in DI estimates. For example in humans bisphenol A (BPA), like other phenols, is expected to be excreted primarily via active tubular secretion in the kidneys (Mahalingaiah et al., 2008). It could also be noted that glomerular filtration rate should be interpreted in the context of physiological effects such as pregnancy and therefore may not be appropriate to use creatinine correction in these subpopulations. 
Table 4 DI equations based on various approaches to adjust for the urine dilution effects

a Based on urine volume adjustment approach

$$
D I_{V}=\frac{C_{\text {volumetric }}\left(\frac{\mu \text { moles }}{L}\right) \times V_{24 h}\left(\frac{L}{\text { day }}\right) \times M_{p}}{\text { Bodyweight }(K g) \times F U E}
$$

b Based on creatinine adjustment approach

$$
D I_{C r}=\frac{C_{C r \text { adjusted }}\left(\frac{\mu \text { moles }}{g \text { cr }}\right) \times C E R\left(\frac{g C r}{d a y}\right) \times M_{p}}{\text { Body weight }(K g) \times F U E}
$$

c Based on urine osmolality adjustment approach

$$
\begin{aligned}
& C_{\text {Osm-adj }}\left(\frac{\mu \text { moles }}{L}\right)=\frac{C\left(\frac{\mu m o l e s}{L}\right) \times \text { median Osmolality }\left(\frac{\text { Osm }}{K g}\right)}{\text { Osmolality }_{\text {specimen }}\left(\frac{\text { Osm }}{K g}\right)} \\
& D I_{\text {Osm }}=\frac{C_{O s m}\left(\frac{\mu m o l e s}{L}\right) \times V_{24 h}\left(\frac{L}{\text { day }}\right) \times M_{p}}{\text { Bodyweight }(K g) \times F U E}
\end{aligned}
$$

d Based on urine specific gravity adjustment approach

$$
\begin{aligned}
& C_{S G-\text { adj }}\left(\frac{\mu \text { moles }}{L}\right)=\frac{C\left(\frac{\mu \text { moles }}{L}\right) \times\left(S G_{\text {ref }}-1\right)}{\left(S G_{\text {specimen }}-1\right)} \\
& D I_{S G}=\frac{C_{S G-\text { adj }}\left(\frac{\mu \text { moles }}{L}\right) \times V_{24 h}\left(\frac{L}{\text { day }}\right) \times M_{p}}{\text { Bodyweight }(K g) \times F U E} \\
& V_{24}=\text { Urinary flow rate }\left(\frac{m L}{\text { hour }}\right) \times 24
\end{aligned}
$$

Notes: Legends: $D I_{V}=\mathrm{DI}$ estimate using urine volume approach $(\mu \mathrm{g} / \mathrm{kg}-\mathrm{bw} / \mathrm{d})$; $D I_{C r}=$ DI estimate using creatinine correction approach $(\mu \mathrm{g} / \mathrm{kg}-\mathrm{bw} / \mathrm{d}) ;$

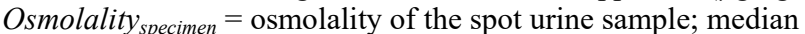
osmolality = median osmolality of all spot samples; $D I_{O s m}=$ DI estimate using urine osmolality approach ( $\mu \mathrm{g} / \mathrm{kg}$-bw/d); $S G_{r e f}=$ reference specific gravity (e.g., mean) of all samples; $D I_{S G}=$ DI estimate using urine specific gravity adjustment approach $(\mu \mathrm{g} / \mathrm{kg}-\mathrm{bw} / \mathrm{d}) ; V_{24 h}=$ estimated $24 \mathrm{~h}$ urine volume; $C E R=$ creatinine excretion rate; $M_{p}=$ molecular weight of parent compound; $F U E=$ fractional urinary excretion.

- Osmolality adjustment: urine osmolality reflects an individual's hydration status and is a measure of the number of dissolved particles per unit of water in the urine. It is expressed as milliosmoles per kilogram of water $(\mathrm{mOsm} / \mathrm{kg}$ water). Urine osmolality can range from $50-1,400 \mathrm{mOsm} / \mathrm{kg}$ water, but the average is between $500-800 \mathrm{mOsm} / \mathrm{kg}$ (Frederiksen et al., 2013). On the other hand, urine osmolality is directly related to the number of particles in solution and is unaffected by the molecular weight and size of these particles. Frederiksen et al. (2013) measured urinary phthalate concentrations and used urine osmolality to adjust for urinary dilution. To adjust, the urinary biomarker concentration $(\mathrm{ng} / \mathrm{mL})$ was divided by the 
urinary osmolality (Osm/ $\mathrm{kg}$ water) of that sample and multiplied by the median osmolality of all included urine samples in the study. The osmolality adjusted urine concentration is multiplied by the total urine output (adjustment factor) to estimate DI (see Table 4 for the DI equation).

- Specific gravity-adjustment: urinary specific gravity is a valid indicator of urinary osmolality - the 'gold standard' measurement of soluble particle concentrations in urine (Chadha et al., 2001). Several studies have shown that the specific gravity-adjustment method for correcting urine dilution introduced less variability to datasets when compared to the creatinine-adjustment method (Ikeda et al., 2003; Miller et al., 2004; Pearson et al., 2009). The main assumption in specific gravity-adjustment is that changes in urinary flow rate do not affect the relative ratio between the mass of the xenobiotic and the mass of the total dissolved solids (SG) in a urinary spot sample (Pearson et al., 2009). The ratio of the amount of biomarker and total dissolved solids remains constant as urinary flow fluctuates throughout the day. Like osmolality method, The SG adjusted biomarker concentration is multiplied by the total urine output (adjustment factor) to estimate DI (Table 4) (Cone et al., 2009; Koch et al., 2014).

\subsubsection{DI estimation for chemicals with multiple metabolites}

Often human exposure to an environmental chemical could lead to the formation of more than one metabolite in urine which is measured in HBM studies. For example, controlled human exposure studies using labelled standards showed that DEHP is metabolised to several primary and secondary metabolites such as MEHP, MEOHP, MEHHP, MECPP and MCMHP (Koch et al., 2005; Silva et al., 2006). The number of metabolites produced for a given parent chemical depends on its PK characteristics (which are in turn governed by its physicochemical properties) as well as the thermodynamic and/or kinetic stability of the metabolites.

The FUE values for the metabolites of DEHP (see Table 3) suggest that none of them can be considered as a 'major metabolite'. Therefore, it is recommended to sum the urinary metabolite concentrations (and the FUEs) before proceeding to compute DI estimates. The DEHP DI can be estimated by using the individual concentration of the metabolite (and corresponding FUE values) in equationslisted in Table 4. For example, DI of DEHP has been estimated using the sum of the concentrations of three metabolites (Wittassek et al., 2007a; Hines et al., 2011) and five metabolites (Wittassek et al., 2007b; Frederiksen et al., 2011) in population level biomonitoring studies.

\subsubsection{Estimates for blood biomarkers}

DI estimates for several persistent organic pollutants (POPs) such as perfluorooctanoic acid (PFOA), perfluorooctanoic sulfonic acid (PFOS), and PBDE have been estimated using measured blood concentrations (Fromme et al., 2007; Trudel et al., 2008; Thompson et al., 2010; Lorber and Egeghy, 2011). By their nature, POPs are metabolised and eliminated very slowly. Therefore, most of the biomonitoring studies measure the concentration of such compounds in blood. Moreover, owing to their long half-lives (typically many years), the variations due to daily exposures are smoothed out. A simple one-compartment PK model with first order clearance has been used to derive dose estimates for POPs using their concentrations in blood (Lorber and Egeghy, 2011). 
Assuming steady-state exposure conditions, the DI of POPs can be estimated from their concentration in blood using the following equation:

$$
D=k \times V_{d} \times C_{p}
$$

$D$ daily absorbed dose (ng/kg bw/day)

$C_{p}$ serum concentration $(\mathrm{ng} / \mathrm{ml})$ of the target chemical

$V_{d}$ volume of distribution $(\mathrm{ml} / \mathrm{kg} \mathrm{bw})$

$k$ first-order elimination rate in the body $\left(\right.$ day $\left.^{-1}\right)$.

Thus two PK parameters, namely, the first-order elimination rate constant $(k)$ and the volume distribution $\left(V_{d}\right)$, are chemical specific and hence no default values exist. For most of the chemicals, the $V_{d}$ parameter in humans is not known. In such cases, the $V_{d}$ could be calculated using tissue/blood partition coefficient measured in animals (Clewell et al., 2008). As equation (5) indicates, variability in $V_{d}$ estimate have significant effect on the DI estimates. The uncertainty in the DI has been handled differently by different research groups (Trudel et al., 2008; Thompson et al., 2010; Egeghy nd Lorber, 2011).

Like POPs, for compounds with intermediate half-lives (ranging from days to month; e.g., methyl mercury), DI can be estimated using a single-compartment model. However, in such cases the temporal variability and uncertainty in the dose estimates would be substantially higher than in the case of POPs (Clewell et al., 2008). Although similar DI estimates for non-persistent chemicals (half-life in the order of hours) using blood biomarkers are possible, such estimation is prone to severe temporal variability and will reflect only recent exposures.

\subsection{Probabilistic approaches}

Probabilistic reverse dosimetry approaches require strong computational capabilities. This method uses validated PBPK modelling and exposure models along with Monte Carlo analysis to predict exposure distributions (Georgopoulos et al., 2009). Different probabilistic approaches have been used for the dose distributions for different chemicals. The exposure conversion factor (ECF) approach is conceptually straightforward. It was proposed by Tan and coworkers (Tan et al., 2007) and assumes that the relationship between a biomarker and dose can be approximated by a linear function for exposure reconstruction purposes. The detailed methodology involved in the probabilistic approaches are beyond the scope of this review article and the readers are directed to related publications in this area (Clewell et al., 2008; Tan and Clewell, 2010; Tan et al., 2012; Grulke et al., 2013; Côté et al., 2014). This approach has been used to derive exposure distributions to several VOCs such as chloroform bromodichloromethane, dibromochloromethane, and bromoform using NHANES III dataset (Tan et al., 2007; Lyons et al., 2008; McNally et al., 2012).

\subsection{Strengths and limitations of reverse dosimetric approaches}

The approaches reviewed in Section 7 represent simple ways of deriving DI (dose) from the urinary and blood concentrations of chemicals and/or metabolites in an individual, as well as at population levels. These methods provide simple first approximation of 
exposure estimates without any consideration to exposure routes, pattern of exposure and frequency of exposures relative to half-life. Urine flow-rate and osmolality information is increasingly available from large-scale biomonitoring studies which could help estimate intake rates more accurately (Hays et al., 2015). Steady-state conditions are assumed in these models which may not always be true in real-life scenarios. Moreover, in some cases, owing to complicated PK of the chemicals, the use of simple one-compartment models may not be appropriate. Apparent volume distribution and elimination half-life values for many chemicals are not readily available which limits the applicability of models used for blood biomarkers. These approaches use point estimates for PK parameters (e.g., FUE and elimination half-life) and do not take into account uncertainty and/or variability among, for example, different age groups in a given population. Moreover, it also assumes that these input parameters are constant irrespective of the route of exposure.

\section{Sources of variability in biomarker concentrations}

Several studies have assessed the intra- and inter-individual variability in biomonitoring data collected on volunteers ( $\mathrm{Li}$ et al., 2010; Preau et al., 2010; Ye et al., 2011; Fisher et al., 2015), general population studies (Aylward et al., 2012, 2014; Hays et al., 2015) and occupational health studies (Tardif et al., 2002; Berthet et al., 2009). There are a range of factors which produce variability in the concentration of a biomarker and a clear understanding of these factors is critical for the proper interpretation of HBM data in health-risk context. These factors could be broadly classified into biological factors, exposure factors and sampling and analytical methodology factors. We have discussed some of these factors in other parts of the review (for example, Section 4.1). In this section, these factors are elaborated to provide further understanding of variability in biomonitoring data.

\subsection{Biological factors}

Differences in the PK (absorption, distribution, metabolism and excretion) of chemicals between individuals, and within individuals over time, can affect the ways in which humans react to exposure to environmental chemicals. For example, the fraction of the exposed dose that is absorbed by the body could be different among individuals owing to differences in absorption processes (e.g., transport proteins), the route of exposure (e.g., oral versus dermal), age (e.g., neonate, infants, children etc.) and other factors. Such variations in the absorbed dose are expected to result in differences in the measured biomarker concentration in the biological media or tissue sampled (Tardif et al., 2002; Clewell et al., 2004; Berthet et al., 2009; Catherine and Pierre-Olivier, 2009; Spaan et al., 2010).

The volume of distribution $\left(V_{d}\right)$ relates the concentration of the chemical in blood to the dose and in general, the larger the $V_{d}$ for a chemical, the lower its blood concentration. Compounds that are lipophilic, such as dioxins, tend to accumulate in adipose tissues (and hence have relatively lower $V_{d}$ ). As a result, biological factors that tend to affect the volume of adipose tissues (such as obesity) could lower the concentration of lipophilic chemicals in the blood or urine. With the assumption of steady-state, the intake rate is assumed to be equal to the elimination rate. However, in 
BE determination based on the chemical concentration in lipids, the volume of distribution $\left(V_{d}\right)$ is likely to be a major determinant and its variability between individuals will directly impact the interpretation. Moreover, it is important to note that factors that influence lipid-water solubility as well as protein binding (e.g., to serum albumen or alpha 1 acid glycoprotein) will influence the $V_{d}$ and impact BE calculations (Clewell et al., 2008).

Variation in clearance processes can also influence biomarker concentration in some cases, particularly when the chemical is cleared from the body through multiple pathways, for example via pulmonary/biliary/renal/metabolic pathways (Epstein, 1996; Mühlberg and Platt, 1999). Metabolism of a parent compound to more water soluble metabolites through oxidative (phase 1) or conjugative (phase 2) metabolic steps provides the opportunity for variation based on expression or activity of the enzymes as well as pharmacogenomic differences (Clewell et al., 2004). Metabolic clearance varies among different groups due to several factors (e.g., age, gender, genetic factors, anthropometric differences, lifestyle, pregnancy/lactation, pathological conditions, or polymorphism) which might be important in understanding the overall variability in the biomarker data (Clewell et al., 2004; Catherine and Pierre-Olivier, 2009).

For chemicals that are eliminated in part by renal mechanisms, the variation between and within individuals in the fraction of the dose appearing in the urine will influence urinary concentrations. Consequently, variability in the FUE of chemicals will impact the interpretation of urinary biomarker data. Sandborgh-Englund et al. (2006) assessed the elimination kinetics of triclosan in humans and found that the triclosan FUE varied from 0.24 to 0.83 with a median of 0.54 . Such variations would have a direct impact on the DI calculations. While it is beyond the scope of this paper, computational methods are available to explore the impact of these types of variations in the biomonitoring data interpretation (see for example Clewell et al., 2008).

Other factors that induce variability in DI calculations include various adjustments to calculate the daily excretion rates. In the absences of relevant data, default values are often used for key physiological parameters, for example CER and total urine output, while estimating DIs. Usage of default values is another source of variability in DI estimation and should be taken into consideration when comparing DI estimates among different studies.

\subsection{Exposure-related factors}

Most of the biomonitoring data currently available in population representative surveys such as CHMS and NHANES are exposure biomarkers that are useful in exposure assessment. In this regard and as discussed in Section 4.1, choice of appropriate biomarker(s) (in terms of sensitivity and specificity as well as potential relevance to the MOA) is critical for exposure assessment. In addition, the frequency of exposure and PK characteristics such as the half-life of the biomarker could significantly influence the measured concentration in bio-specimens. For persistent compounds (with half-lives in the order of years, such as dioxins) the temporal fluctuations in biomarker concentrations would be negligible. In such cases, variations in exposure frequency are expected to have minimal influence on the biomarker concentration (Aylward et al., 2012). However, for chemicals with short half-lives (e.g., hours), the exposure frequency will have a significant influence in determining the temporal variability of the biomarker concentration in blood or urine (Koch et al., 2014). 
In addition, concentrations in spot urine samples from diverse individuals are expected to vary owing to differences in the exposure patterns (between-person variability). Chemicals such as triclosan and phthalates are found in several consumer and personal care products and the use pattern of products varies significantly between individuals. In an observational and interventional study, Koch and co-workers assessed the intra- and inter-individual variability in the urinary concentration of triclosan, parabens, triclocarban and benzophenones in eight individuals over a period of six days (Koch et al., 2014). All participants provided complete urine voids during the period of the study as well as maintaining a diary of personal care product (e.g., toothpaste) use during those days. Moreover, in two out of the six days participants were provided with replacement personal care products which did not contain the target analytes. They observed that the concentration of target compounds in urine samples collected during the replacement product use days are relatively lower compared to other days. Moreover, preliminary analysis showed that the product use diary data correspond well with the observed urinary concentrations of the target analytes. Thus differences in the exposure patterns among individuals are expected to introduce variability in the biomonitoring data.

Similarly, Preau et al. (2010) studied the temporal variability in urinary phthalate metabolite concentrations using data collected from eight adult volunteers. Specific metabolites of diethyl phthalate [DEP; metabolite: mono-ethyl phthalate (MEP)] and di (2-ethylhexyl) phthalate [DEHP; metabolite: (2-ethyl-5-hydroxyhexyl) phthalate (MEHHP)] were measured in the urine samples (spot samples, morning void and $24 \mathrm{~h}$ composite sample) using a validated analytical method. The results indicated that regardless of the type of void (spot samples, morning void and $24 \mathrm{~h}$ composite sample), the inter-person variability in the concentration accounted for $>75 \%$ of the total variability in the MEP concentration. On the other hand, within-person variability contributed to about $70-80 \%$ of the total variability in the MEHHP concentration. The general population exposure to DEP occurs mainly through the use of consumer and personal care products while DEHP exposures occur mainly via food (Preau et al., 2010). The authors noted that the differences in the variability contributors (inter-person versus within-person) in MEP and MEHHP correlate well with the use patterns of the respective phthalates. However, as mentioned earlier, data from nationally representative surveys such as CHMS are affected to a lesser extent by the inter- and intra-day variability due to their large sample size.

\subsection{Sample collection and analytical methodology factors}

The sample collection protocols used in biomonitoring studies could have a significant effect on the biomonitoring data. For example, in cycle 1 of the CHMS 2007-2009 (Health Canada, 2010), some of the participants were requested to fast prior to the collection of bio-specimens. This would affect the concentrations of biomarkers (chemicals) for which food is the major source of exposure in humans (e.g., di(2-ethylhexyl) phthalate or DEHP) (Koch and Calafat, 2009; Saravanabhavanet al., 2013). For some chemicals, sampling time may affect the measured biomarker concentrations. For example, Silva et al. (2006) observed that the concentrations of some of the phthalate metabolites were significantly higher in individuals who provided urine samples in the evening compared to individuals providing samples in the morning. In this 
particular case, such variability is attributed to the short half-life of phthalate metabolites and the exposure patterns.

Ensuring the analytical quality of the biomarker measurement is important and discussed in Section 2.1. Developing and employing appropriate QA/QC system is critical to reduce analytical variability and to ascertain accuracy and long-term precision of the biomonitoring data. In addition, care must be taken to avoid contamination of samples during sample storage, processing and analysis to avoid erroneous results. For example, external contamination could occur from the use of contaminated solvents and/or reagents, experimental apparatus and laboratory environment, although pinning down the specific contamination source is often very difficult and depends on the nature of the biomarker that is being analysed (Ye et al., 2013). Even if the biomonitoring data are generated in a laboratory with proper QA/QC protocols in place, biomonitoring data produced in two different laboratories must be compared with caution due to interlaboratory and inter-methodology differences (Needham et al., 2007; Esteban et al., 2014). To improve the comparability of HBM data, it is highly desirable for harmonising HBM methodologies. In Europe such harmonisation exercise for sampling design, survey protocols and laboratory methods were carried out recently as a part of DEMOCOPHES project (Schindler et al., 2014; Casteleyn et al., 2015). As a part of harmonisation of laboratory methods, 38 analytical laboratories from 17 member states of European Union participated in two inter-laboratory comparison exercises and in two external quality assessment exercises in which they analysed five groups of target analytes (Schindler et al., 2014). Ten internationally reputed analytical laboratories served as reference laboratories for external quality assessment. Clearly the above example illustrates the importance of collaboration and understanding amongst analytical laboratories involved in biomonitoring studies. Such collaborative exercises would also help to build analytical capacity and would educate new laboratories on best practices in HBM methodologies (Katsonouri et al., 2015).

\section{Integration of HBM data in human health risk assessment}

So far, we have broadly reviewed the nature of the HBM data and tools required for its interpretation in the health-risk context. In this section, examples are presented to illustrate how these tools are being used to integrate HBM data in traditional risk assessment paradigm. Lead and mercury are two chemicals for which several epidemiological studies have directly linked human biomonitoring data to adverse-health outcome (Kjellström et al., 1986, 1989; Grandjean et al., 1997; Canfield et al., 2003; Lanphear et al., 2005; Jedrychowski et al., 2007).

Such biomarker-health effects relationship formed the basis of risk assessment and risk management activities for these chemicals (CDC, 2005; Alarcon et al., 2011; Health Canada, 2013a). Therefore, there is no need for additional tools for integrating lead and mercury HBM data in the health-risk context. Risk assessment practitioners have identified a few other chemicals (e.g., ethanol) to have sufficient biomarker-health effects data (Maier et al., 2004). However, developing a robust biomarker-health effect relationship is highly resource intensive and time consuming. The majority of chemicals for which biomonitoring data currently exist (Health Canada, 2013b; CDC, 2015), do not have robust biomarker-health effects relationships. Therefore, as a practical measure, use 
of tools described in previous sections for integrating HBM data in health risk assessment appears logical.

\subsection{Applications of forward dosimetry tools in human health risk assessment}

Aylward et al. (2013) recently utilised BE approach to generate chemical specific HQs for 130 chemicals by comparing the BEs to the corresponding biomonitoring data from CDC (CDC, 2015). In most cases the data from the descriptive statistics published by $\mathrm{CDC}$ were used. Additional descriptive statistics were created for analytes which have been shown to have dependency on age or smoking status (e.g., acrylamide). Among the chemicals that were not part of the volatile organic group (VOCs), the 95th percentile population level biomarker concentration approached or exceeded the HQ value of 1 for acrylamide (in smokers), DEHP, dioxins, cadmium (smokers), and arsenic. For 11 chemicals comparison of the GM biomarker concentrations with BEs resulted in HQ values between 0.1 and 1 . Among the VOC group, the 95th percentile concentration for benzene (smokers), xylenes (both smokers and non-smokers), and 1,4-dichlorobenzene exceeded HQ of 1. Like DEHP, most of the VOCs included in this analysis have a short half-life. Therefore, the authors noted that for these compounds use of 95th percentile concentrations may not represent daily or long-term average exposure level. In such cases, comparison of GM or other central tendency measures would be meaningful for risk assessment. Five analysed VOCs at GM concentrations produced a HQ between 0.1 and 1 . For 12 out of 13 chemicals that have cancer risk-based screening values, both GM and 95th percentile concentrations exceeded $1 \times 10^{-6}$ risk level. For eight chemicals the GM concentration exceeded $1 \times 10^{-4}$ risk level. However, since BE values were generated as steady-state concentrations at RsD for each chemical, this analysis provides a worst-case exposure scenario and should not be generalised.

A similar analysis for 17 chemicals using biomonitoring data from CHMS cycle 1 and cycle 2 was presented (St-Amand et al., 2014). The BE values based on Health Canada exposure guidance values were used wherever available. For the majority of the chemicals analysed, the HQ values evaluated at the 95th percentile concentration of the biomarkers did not exceed 1 . Among the ten chemicals (out of 17) with relatively short half-life $(<1 \mathrm{~d})$, HQ calculated using GM concentration of arsenic was between 0.1 and 1. For rest of the chemicals, HQ calculated based on the GM of the concentrations was between 0.001 and 0.1. Like NHANES study, the HQ for arsenic calculated based on 95th percentile exceeds 1 . However, the HQ for DEHP even at 95th percentile did not exceed 1. This may be partly due to the fact that a different BE value for DEHP, based on a Health Canada exposure guidance value and three metabolites, was used in this analysis.

In the case of chemicals with long half-life (seven out of 17), the HQ for all chemicals, with the exception of cadmium, calculated at the GM did not exceed 0.1. The HQ for PCBs calculated at 95 th percentile fell between 0.1 and 1 . In the case of cadmium the HQ calculated at 95th percentile was approaching or exceeding 1 while HQ calculated at GM was between 0.1 and 1 . The cancer risk was evaluated for arsenic, dichlorodiphenyltrichloroethane (DDT) and hexachlorobenzene. The cancer risk for DDT and hexachlorobenzene was between $1 \times 10^{-5}$ and $1 \times 10^{-6}$. The cancer risk for inorganic arsenic exceeded $1 \times 10^{-4}$; however its predominant metabolite, dimethyl arsenic (DMA), could be formed from other organic arsenic species and hence the inorganic arsenic exposure estimates are likely overestimated. 
The studies presented here illustrate some recent examples in which the BE approach could be used as a risk assessment screening tool. However, it should be noted that HQ $>1$ does not necessarily mean a risk to the general population is imminent. It simply helps to evaluate exposures and prioritise them for further detailed risk assessment. Moreover, as mentioned earlier, the BE approach is as good as the exposure guidance value upon which it was built and it carries all the assumptions and limitations associated with the development of the exposure guidance value. Finally, since both HBM-1 derived by German Biomonitoring Commission and $\mathrm{BE}$ are in principle the same, HBM-1 values could be used in the same fashion as BE to interpret general population data in the health risk context.

Another example for the utility of biomonitoring data in health risk assessment is provided in the Scientific Committee on Consumer Products (SCCP) opinion on triclosan (European Commission, 2009). Triclosan is a preservative and an antimicrobial agent widely used in cosmetics and consumer products such as tooth paste and mouth wash. In EU the maximum allowable concentration of triclosan is $0.3 \%$. As a part of their scientific opinion, the SCCP presented a triclosan exposure assessment. The systemic exposure dose (SED) was estimated by traditional pathway analysis using data on dermal absorption and oral exposure. The MOS was evaluated by two methods. In the first method, the SED dose was compared with the NOAEL of $12 \mathrm{mg} / \mathrm{kg}$ bw/d from a rat bioassay which resulted in a MOS of 380. In the second method, comparison of serum triclosan data from a human biomonitoring study to the rat triclosan serum data measured at NOAEL dose level resulted in a higher MOS of 940. However, the participants in the human biomonitoring study did not use all common products that contain triclosan (European Commission, 2009). The SCCP used worst-case exposure scenarios in estimating SED exposures. Therefore, to be more conservative, the SCCP used MOS based on SED exposures to derive its conclusion and used MOS based on biomonitoring data as supporting evidence.

As mentioned in Section 5.2, tools such as BEI and BAT are specifically formulated to make decisions on the safe exposure levels in occupational settings. These tools have been developed keeping in mind workers who are supposed to be in good health and taking into account normal work hours (40 h per week). On the other hand, the composition of the general population is strikingly different and contains several vulnerable sub-populations such as infants, pregnant women and the aged populations. Moreover, the general population exposures are averaged for the full day ( $24 \mathrm{~h})$ (NRC, 2006). Although it is intuitive to think that the occupational values could be extrapolated to the general population after applying UF, no formal guidelines (or criteria) are currently available for such extrapolation. Therefore the use of these tools in general population risk assessment is very limited (ACGIH, 2015).

\subsection{Applications of reverse dosimetry tools in human health risk assessment}

In March 2012, Health Canada and Environment Canada jointly published a preliminary risk assessment report on triclosan (Health Canada, 2012). In this work, Health Canada has extensively used human biomonitoring data to estimate triclosan exposure in the general population. Since at that time, no CHMS data on triclosan was available, biomonitoring data from NHANES was used as a surrogate. There are numerous monitoring and surveillance initiatives funded under Canada's Chemicals Management Plan that will be included in the final risk assessment report on triclosan including the 
second report on human biomonitoring of environmental chemicals in Canada (data from CHMS cycle 2), the MIREC studies (MIREC, MIREC-ID and MIREC-CD plus), and the Plastics and Personal Care Product Use in Pregnancy study (P4) (Government of Canada, 2014). These initiatives will allow incorporation of Canadian biomonitoring data into the triclosan health risk assessment and provide a better understanding of exposure for the general population in Canada including pregnant women, children and infants.

Using the urine volume approach (cf., Section 6.1.1) the DI rates for the general population ( $>$ six years) were calculated. For children $<$ six years, aggregate exposure was estimated by summing the DIs from urinary concentrations from other biomonitoring studies, concentration from breast milk, and incidental exposures from object-to-mouth activity and hand-to-mouth activity. Since NHANES did not report total urine output for survey participants, the urine output data (mean and 95th percentile for different age groups) from Geigy scientific tables (Lentner, 1981) was used. Overall triclosan DI for the general population aged $\geq$ six years based on mean and 95th percentile urine output data was estimated to be $0.0029 \mathrm{mg} / \mathrm{kg}$ bw/d and $0.0045 \mathrm{mg} / \mathrm{kg}$ bw $/ \mathrm{d}$ respectively. The corresponding margin of exposures (MOEs), estimated by comparing these DI with a NOAEL of $25 \mathrm{mg} / \mathrm{kg}$ bw/d from a mouse study (critical study), were 8,621 and 5,556 respectively. In addition to the overall estimates, the DIs as well as the MOEs for different age groups was also estimated (see Table 4 in Health Canada, 2012)

Recently, the Chronic Hazard Advisory Panel (CHAP) on phthalates and phthalate alternatives used urinary phthalate metabolite data from NHANES 2005-2006 and study for future families (SFF) to estimate DIs of several phthalates in the US general population, in pregnant women (NHANES, SFF) and in infants (CHAP, 2014). The creatinine adjustment approach (cf., Section 6.1.1) was used to estimate phthalate DIs using urinary metabolite data. The CER was estimated in individuals using Mage equations based on the height, weight and sex of the participants (Mage et al., 2008; Huber et al., 2011). Since the height and weight information were not collected in SFF study, a default value for CER applicable to pregnant women was used. FUE values for different phthalate metabolites published in peer-reviewed literature were used to estimate DI (see for example Table 4). The phthalate DI estimates were used to derive HQ for individual phthalates. Cumulative risk assessment for phthalates having similar toxicity was also conducted.

Apart from using HBM data to estimate DIs, CHAP has estimated DI for phthalates using aggregate external exposure approach/scenario-based exposure approach. The average phthalate DIs based on the modelling were in general agreement with those obtained using HBM data. However, for some phthalates, the HBM approach produced slightly higher DI (e.g., DEHP and dibutyl phthalates) compared to modelling. In addition to demonstrating the usefulness of HBM data in the traditional risk assessment, this example illustrate that:

1 HBM data serves as a 'fact check' of exposure estimates produced using modelling approach

2 in contrary to general perception, the modelling approach do not always overestimate exposures as exposures to some of the phthalates using HBM approach were higher.

The US Consumer Product Safety Commission (CPSC) has extended CHAP's methodology to estimate DIs of phthalates in pregnant women and women of reproductive age (15-45 years) based on NHANES data from 2005-2006, 2007-2008, 
2009-2010 and 2011-2012 (CPSC, 2015). In addition to exposure estimates, phthalate risk, and cumulative phthalate risk (based on simultaneous exposure to multiple phthalates) were estimates for individuals and descriptive statistical parameters (e.g., median, and 95th percentile) of the risk estimates for the overall population were computed. Due to a very limited number of pregnant women in NHANES 2007-2008, 2009-2010 and 2011-2012 datasets, stable statistical estimates for phthalate exposure and risk were not calculated for this sub-population. In these cases, estimates were derived only for women of reproductive age. The results from this study showed that the DI estimates for DEHP has decreased in latter cycles of NHANES compared to 2005-2006. On the other hand, the DI estimates for DINP has increased in the latter cycles of which parallels the replacement of DINP for DEHP in the consumer products.

In July 2015, Health Canada and Environment Canada jointly released state of the science reports on some phthalates (Environment Canada, Health Canada, 2015a, 2015b, 2015c, 2015d). They have derived DI estimates for several phthalates based on the respective urinary phthalate metabolite data from the CHMS (cycles 1 and 2), P4, MIREC, MIREC-CD Plus. When appropriate Canadian data were not available, biomonitoring data from NHANES was used. Like CHAP, Health Canada used creatinine correction approach (cf., Section 6.1.1) for deriving DI estimates. The CER s were derived in individuals using Mage equations (Mage et al., 2008; Huber et al., 2011). Median body mass indices were used for the respective population groups in both the CHMS and NHANES analyses. For P4 and MIREC, the median body mass indices for the women in the surveys were used to evaluate the adiposity adjustment. In addition to using HBM data to estimate DIs, Health Canada has estimated DI for phthalates using a scenario-based exposure approach. The average phthalate DIs based on the modelling of environmental media and food exposure were in general agreement with those obtained using HBM data. However, the modelling of exposure via products used by consumers was generally higher than the exposure estimates using the HBM approach.

\section{Confidence assessment}

Biomonitoring data are collected, interpreted, communicated and used in public health decision making activities (Bahadori et al., 2007; Lioy et al., 2015). Consequently, it is essential to characterise the strength of the evidence and robustness of the approaches used in these initiatives. Therefore, uncertainty assessment forms an integral part of biomonitoring data interpretation and communication (Lakind et al., 2008a). Factors that affect variability in HBM data introduce different degrees of uncertainty in the interpretation of biomonitoring data. As these factors are biomarker- and chemicalspecific, the uncertainty surrounding the data interpretation varies between chemicals.

Knowledge about the MOA, the nature of the chemical entity causing the toxicity, the relationship (through metabolic pathway analysis) of the measured biomarker to the actual toxicant are important to interpret HBM data in a risk-based context. For example, conclusions regarding exposure and health effects can be deduced with greater certainty and confidence from the biomonitoring data for data-rich chemicals such as lead and mercury (Kjellström et al., 1986, 1989; Grandjean et al., 1997; Canfield et al., 2003; Lanphear et al., 2005; Jedrychowski et al., 2007). In fact, the biomonitoring data for these chemicals are used to make health policy decisions and interventions (WHO, 2001; CDC, 2005; Lanphear, 2005; NRC, 2006; Alarcon et al., 2011). However, in many instances 
there are gaps in our understanding of the mechanisms of toxicity as well as in our knowledge of toxicokinetic parameters. These gaps in knowledge and understanding increase the uncertainty around our interpretation and lower our confidence concerning the potential population health impacts. Therefore, confidence assessment in the context of incorporation of HBM data in risk assessment should consider factors such as availability of toxicological and epidemiological evidences in animals and humans, MOA of chemicals in animal models and its relevance to humans, human/animal PK data, validation and robustness of PK data/models among others.

\section{Summary and key findings}

Selected key findings from this review are summarised below:

- Detection of reliable biomarker(s) of a given chemical in an appropriate biological specimen provides direct evidence of exposure to that chemical, however, the absence of a measurable concentration of that biomarker does not necessarily indicate the absence of exposure.

- Several factors including the sensitivity and specificity of the biomarker, availability of validated analytical method(s), physicochemical characteristics of the chemical, as well as its PK characteristics affect the selection of biomarkers and the interpretation of biomonitoring results.

- An evaluation of temporal and special variability is critical for the inclusion of biomonitoring data in risk assessment.

- Direct comparison of HBM data with biomonitoring-based toxicological reference values is a straightforward approach to interpreting HBM data. However, such reference values are available for only a few chemicals (e.g., blood lead and blood mercury).

- For chemicals that do not have biomonitoring-based toxicological reference values, different forward and reverse dosimetry-based methods exist for the interpretation of HBM data in a population health and risk assessment context. Matrix-specific methods exist in both forward and reverse dosimetry approaches for interpreting blood and urine biomarker concentration data.

- Currently a handful of examples exist in which the human biomonitoring data has been incorporated into health risk assessment. However this trend is expected to grow in the coming years when more and more biomonitoring data as well as methods to interpret them becomes available.

\section{Knowledge gaps and future directions}

Data from HBM studies have helped to fill key data-gaps in human health risk assessments of environmental chemicals. Examples of human health risk assessments 
provided in Section 8 illustrate the role of HBM in understanding the link between exposure and health effects. The HBM data provides an estimate of integrated exposure. Source attributions using HBM data in the exposure assessments needs concurrent efforts to gather information on possible exposure sources for a given chemical. Thus, a clear understanding of HBM-based approaches is required to assess the extent to which the HBM data could improve health risk assessment. Moreover, this will aid in developing general guidelines for choosing appropriate interpretation tools (forward vs. reverse dosimetry) in instances where HBM data are used as a 'reality check' on exposure assessment and/or risk characterisation. In addition, further understanding of the implications of these changes to risk management is required. For many emerging environmental chemicals, lack of relevant PK data (e.g., FUE) is a bottle-neck in interpreting HBM data in population health risk context. Although some data suggest that infants metabolise (Calafat et al., 2004) some environmental chemicals differently than older age groups, it is still not clear to what extent age-related difference in PKs would influence the choice of biomarkers for HBM studies.

Further research and development work is required to address some of the key issues pertaining to acquiring and interpretation of HBM data. More work is needed to identify specific and sensitive biomarkers for emerging contaminants. Additional in-vitro, in-vivo and epidemiological studies are required to identify MOA, toxicity and dose-response relationships for emerging chemicals and to aid biomarker development. Validity and applicability of these biomarkers in different population sub-groups (e.g., children) need to be tested for wider usage in HBM programs.

Certified/standard reference materials (CRM/SRM) are available for only for a handful of environmental chemicals. Therefore, there is an urgent need for developing new reference materials required to assess the performance of the analytical laboratories. Moreover, there are currently very few inter-laboratory proficiency testing programs [such as the German External Quality Assessment Scheme (G-EQUAS)] that are relevant for human biomonitoring studies. Government agencies could play a key role in developing such programs to assess the quality and comparability of the HBM data produced by different laboratories.

Approaches/methodologies that will improve the current DI estimation methods or simplify the probabilistic methods would be desirable. In addition, approaches that incorporate non-steady state kinetics in both forward and reverse dosimetry approaches would be useful as in real life most environmental exposure the general population occurs intermittently. The FUE values for only very few metabolites/biomarkers are currently available. Novel innovative approaches are required for deriving FUE values for several chemicals in order to derive exposure estimates from biomonitoring data.

Additional research efforts are required to adjust for urine dilution effects in measures of environmental chemicals in spot urine samples. Continuous improvements in PK/PBPK models and their usage for interpreting HBM data could help to overcome some issues pertaining to variability. Moreover, adjustment of urinary metabolite concentrations using osmolality/specific gravity to adjust for urine dilution effects appears to be promising especially when interpreting biomonitoring data from infants. Although the fundamental theory pertaining to osmolality adjustment is well grounded, its application to HBM data interpretation needs further improvement. 


\section{References}

Agency for Toxic Substances and Disease Registry (ATSDR) (2012) Minimum Risk Levels for Hazardous Substances [online] http://www.atsdr.cdc.gov/mrls/mrllist.asp (accessed 2 October 2012).

Agency for Toxic Substances and Disease Registry (ATSDR) (2015) Minimal Risk Levels (MRLs) for Hazardous Substances, ATSDR, Atlanta, GA [online] http://www.atsdr.cdc.gov/mrls/index.asp (accessed 7 January 2016).

Alarcon, W., Graydon, J. and Calvert, G. (2011) 'Adult blood lead epidemiology and surveillance United States, 2008-2009', Morbidity and Mortality Weekly Report, Vol. 60, No. 25, pp.841-845.

Alavanja, M.C., Sandler, D.P., McMaster, S.B., Zahm, S.H., McDonnell, C.J., Lynch, C.F., Pennybacker, M., Rothman, N., Dosemeci, M., Bond, A.E. and Blair, A. (1996) 'The agricultural health study', Environmental Health Perspectives, Vol. 104, No. 4, pp.362-369.

Alberta Health and Wellness (AHW) (2008) Alberta Biomonitoring Program: Chemicals in Serum of Pregnant Women in Alberta, Public Health Division, Alberta Health and Wellness, Edmonton, Alberta [online] http://www.health.alberta.ca/documents/Chemical-Biomonitoring2008.pdf (accessed 7 January 2016).

Alberta Health and Wellness (AHW) (2010) Alberta Biomonitoring Program: Chemicals in Serum of Children in Southern Alberta 2004-2006. Influence of Age and Comparison to Pregnant Women, Public Health Division, Alberta Health and Wellness, Edmonton, Alberta [online] http://www.health.alberta.ca/documents/Chemical-Biomonitoring-2010.pdf (accessed 7 January 2016).

Albertini, R., Bird, M., Doerrer, N., Needham, L., Robison, S., Sheldon, L. and Zenick, H. (2006) 'The use of biomonitoring data in exposure and human health risk assessments', Environmental Health Perspectives, Vol. 114, No. 11, pp.1755-1762.

American Conference of Governmental Industrial Hygienists (ACGIH) (2015) TLVs and BEIs: Threshold Limit Values for Chemical Substances and Physical Agents\& Biological Exposure Indices, ACGIH Worldwide, Cincinnati, $\mathrm{OH}$.

Anderson, W.A.C., Castle, L., Scotter, M.J., Massey, R.C. and Springall, C. (2001) 'A biomarker approach to measuring human dietary exposure to certain phthalate diesters', Food Additives and Contaminants: Part A, Vol. 18, No. 12, pp.1068-1074.

Angerer, J. (2012) 'Strengths and limitations of HBM - yes we can!', Int J Hygiene and Environmental Health, Vol. 215, No. 2, pp.96-97.

Angerer, J., Aylward, L.L., Hays, S.M., Heinzow, B. and Wilhelm, M. (2011) 'Human biomonitoring assessment values: approaches and data requirements', Int J. Hygiene and Environmental Health, Vol. 214, No. 5, pp.348-360.

Angerer, J., Bird, M.G., Burke, T.A., Doerrer, N.G., Needham, L., Robison, S.H., Sheldon, L. and Zenick, H. (2006) 'Strategic biomonitoring initiatives: moving the science forward', Toxicological Sciences, Vol. 93, No. 1, pp.3-10.

Angerer, J., Ewers, U. and Wilhelm, M. (2007) 'Human biomonitoring: state of the art', Int J. Hygiene and Environmental Health, Vol. 210, No. 3, pp.201-228.

Arbuckle, T.E., Davis, K., Marro, L., Fisher, M., Legrand, M., LeBlanc, A. and MIREC Study Group (2014) 'Phthalate and bisphenol A exposure among pregnant women in Canada results from the MIREC study', Environment International, Vol. 68, pp.55-65.

Arbuckle, T.E., Fraser, W.D., Fisher, M., Davis, K., Liang, C.L., Lupien, N. et al. (2013) 'Cohort profile: the maternal-infant research on environmental chemicals research platform', Paediatric and Perinatal Epidemiology, Vol. 27, No. 4, pp.415-425.

Arbuckle, T.E., Marro, L., Davis, K., Fisher, M., Ayotte, P., Bélanger, P. et al. (2015) 'Exposure to free and conjugated forms of bisphenol A and triclosan among pregnant women in the MIREC cohort', Environmental Health Perspectives, Vol. 123, No. 4, pp.277-284. 
Arctic Monitoring and Assessment Programme (AMAP) (2009) Human Health in the Arctic, Arctic Monitoring and Assessment Programme, Oslo, Norway [online] http://www.amap.no/ documents/doc/amap-assessment-2009-human-health-in-the-arctic/98 (accessed 7 January 2016).

Arnold, S.M., Angerer, J., Boogaard, P.J., Hughes, M.F., O’Lone, R.B., Robison, S.H. and Robert Schnatter, A. (2013) 'The use of biomonitoring data in exposure and human health risk assessment: benzene case study', Critical Reviews in Toxicology, Vol. 43, No. 2, pp.119-153.

Aylward, L.L. and Hays, S.M. (2008) 'Biomonitoring equivalents (BE) dossier for 2, 4-dichlorophenoxyacetic acid (2, 4-D) (CAS No. 94-75-7)', Regulatory Toxicology and Pharmacology, Vol. 5, No. 3, pp.S37-S48.

Aylward, L.L., Barton, H.A. and Hays, S.M. (2008a) 'Biomonitoring equivalents (BE) dossier for toluene (CAS No. 108-88-3)', Regulatory Toxicology and Pharmacology, Vol. 51, No. 3, pp.S27-S36.

Aylward, L.L., LaKind, J.S. and Hays, S.M. (2008b) 'Derivation of biomonitoring equivalent (BE) values for 2, 3, 7, 8-tetrachlorodibenzo-p-dioxin (TCDD) and related compounds: a screening tool for interpretation of biomonitoring data in a risk assessment context', $J$ Toxicology and Environmental Health, Part A, Vol. 71, No. 22, pp.1499-1508.

Aylward, L.L., LaKind, J.S. and Hays, S.M. (2008c) 'Biomonitoring Equivalents (BE) dossier for trihalomethanes', Regulatory Toxicology and Pharmacology, Vol. 51, No. 3, pp.S68-S77.

Aylward, L.L., Hays, S.M., Gagné, M. and Krishnan, K. (2009a) 'Derivation of biomonitoring equivalents for di (2-ethylhexyl) phthalate (CAS No. 117-81-7)', Regulatory Toxicology and Pharmacology, Vol. 55, No. 3, pp.249-258.

Aylward, L.L., Hays, S.M., Gagné, M. and Krishnan, K. (2009b) 'Derivation of biomonitoring equivalents for di-n-butyl phthalate (DBP), benzylbutyl phthalate (BzBP), and diethyl phthalate (DEP)', Regulatory Toxicology and Pharmacology, Vol. 55, No. 3, pp.259-267.

Aylward, L.L., Hays, S.M., Gagné, M., Nong, A. and Krishnan, K. (2010) 'Biomonitoring equivalents for hexachlorobenzene', Regulatory Toxicology and Pharmacology, Vol. 58, No. 1, pp.25-32.

Aylward, L.L., Hays, S.M., Smolders, R., Koch, H.M., Cocker, J., Jones, K. et al. (2014) 'Sources of variability in biomarker concentrations', $J$ Toxicology and Environmental Health, Part B, Vol. 17, No. 1, pp.45-61.

Aylward, L.L., Kirman, C.R., Adgate, J.L., McKenzie, L.M. and Hays, S.M. (2012) 'Interpreting variability in population biomonitoring data: role of elimination kinetics', $J$ Exposure Science and Environmental Epidemiology, Vol. 22, No. 4, pp.398-408.

Aylward, L.L., Kirman, C.R., Blount, B.C. and Hays, S.M. (2010) 'Chemical-specific screening criteria for interpretation of biomonitoring data for volatile organic compounds (VOCs) application of steady-state PBPK model solutions', Regulatory Toxicology and Pharmacology, Vol. 58, No. 1, pp.33-44.

Aylward, L.L., Kirman, C.R., Schoeny, R., Portier, C.J. and Hays, S.M. (2013) 'Evaluation of biomonitoring data from the CDC national exposure report in a risk assessment context: perspectives across chemicals', Environmental Health Perspectives, Vol. 121, No. 3, pp.287-294.

Aylward, L.L., Krishnan, K., Kirman, C.R., Nong, A. and Hays, S.M. (2011) 'Biomonitoring equivalents for deltamethrin', Regulatory Toxicology and Pharmacology, Vol. 60, No. 2 , pp.189-199.

Bahadori, T., Phillips, R.D., Money, C.D., Quackenboss, J.J., Clewell, H.J., Bus, J.S. et al. (2007) 'Making sense of human biomonitoring data: findings and recommendations of a workshop', $J$ Exposure Science and Environmental Epidemiology, Vol. 17, No. 4, pp.308-313.

Barr, D.B., Wilder, L.C., Caudill, S.P., Gonzalez, A.J., Needham, L.L. and Pirkle, J.L. (2005) 'Urinary creatinine concentrations in the US population: implications for urinary biologic monitoring measurements', Environmental Health Perspectives, Vol. 113, No. 2, pp.192-200. 
Bartoníček, V. (1962) 'Metabolism and excretion of trichloroethylene after inhalation by human subjects', British J Industrial Medicine, Vol. 19, No. 2, pp.134-141.

Becker, K., Schulz, C., Kaus, S., Seiwert, M. and Seifert, B. (2003) 'German Environmental Survey 1998 (GerES III): environmental pollutants in the urine of the German population', International Journal of Hygiene and Environmental Health, Vol. 206, No. 1, pp.15-24.

Becker, R.A., Hays, S.M., Robison, S. and Aylward, L.L. (2012) 'Development of screening tools for the interpretation of chemical biomonitoring data', J Toxicology, Article ID 941082.

Berthet, A., de Batz, A., Tardif, R., Charest-Tardif, G., Truchon, G., Vernez, D. and Droz, P.O. (2009) 'Impact of biological and environmental variabilities on biological monitoring - an approach using toxicokinetic models', Journal of Occupational and Environmental Hygiene, Vol. 73, No. 3, pp.177-184.

Boeniger, M.F., Lowry, L.K. and Rosenberg, J. (1993) 'Interpretation of urine results used to assess chemical exposure with emphasis on creatinine adjustments: a review', American Industrial Hygiene Association Journal, Vol. 54, No. 10, pp.615-627.

Boettcher, M.I., Bolt, H.M., Drexler, H. and Angerer, J. (2006) 'Excretion of mercapturic acids of acrylamide and glycidamide in human urine after single oral administration of deuteriumlabelled acrylamide', Archives of Toxicology, Vol. 80, No. 2, pp.55-61.

Bushnik, T., Haines, D., Levallois, P., Levesque, J., van Oostdam, J. and Viau, C. (2010) 'Lead and bisphenol A concentrations in the Canadian population', Health Reports, Vol. 21, No. 3, pp.7-18.

Calafat, A.M. and McKee, R.H. (2006) 'Integrating biomonitoring exposure data into the risk assessment process: phthalates [diethyl phthalate and Di (2-ethylhexyl) phthalate] as a case study', Environmental Health Perspectives, Vol. 114, No. 11, pp.1783-1789.

Calafat, A.M. and Needham, L.L. (2008) 'Factors affecting the evaluation of biomonitoring data for human exposure assessment', Int J Andrology, Vol. 31, No. 2, pp.139-143.

Calafat, A.M., Needham, L.L., Silva, M.J. and Lambert, G. (2004) 'Exposure to di-(2-ethylhexyl) phthalate among premature neonates in a neonatal intensive care unit', Pediatrics, Vol. 113, No. 5, pp.e429-e434.

Canfield, R.L., Henderson Jr., C.R., Cory-Slechta, D.A., Cox, C., Jusko, T.A. and Lanphear, B.P. (2003) 'Intellectual impairment in children with blood lead concentrations below $10 \mu \mathrm{g}$ per deciliter', New England J Medicine, Vol. 348, No. 16, pp.1517-1526.

Casteleyn, L., Dumez, B., Becker, K., Kolossa-Gehring, M., Den Hond, E., Schoeters, G. et al. (2015) 'A pilot study on the feasibility of European harmonized human biomonitoring: strategies towards a common approach, challenges and opportunities', Environmental Research, Vol. 141, pp.3-14.

Catherine, T. and Pierre-Olivier, D. (2009) 'Age differences in biological monitoring of chemical exposure: a tentative description using a toxicokinetic model', Int Archives of Occupational and Environmental Health, Vol. 82, No. 5, pp.669-676.

Centers for Disease Control and Prevention (CDC) (2005) Preventing Lead Poisoning in Young Children, CDC, Atlanta, GA [online] http://www.cdc.gov/nceh/lead/publications/ PrevLeadPoisoning.pdf (accessed 7 January 2016).

Centers for Disease Control and Prevention (CDC) (2009) Fourth National Report on Human Exposure to Environmental Chemicals [online] http://www.cdc.gov/exposurereport/ (accessed 7 January 2016).

Centers for Disease Control and Prevention (CDC) (2010) 'Vital signs: nonsmokers' exposure to second hand smoke - United States, 1999-2008', Morbidity and Mortality Weekly Report, Vol. 59, No. 35, pp.1141-1146.

Centers for Disease Control and Prevention (CDC) (2015) Fourth National Report on Human Exposure to Environmental Chemicals: Updated Tables, CDC, Atlanta, GA [online] http://www.cdc.gov/exposurereport/ (accessed 7 January 2016). 
Černá, M., Batáriová, A., Šmíd, J., Čejchanová, M., Očadlíková, D., Bavorová, H. and Beneš, B. (2007) 'Human biomonitoring system in the Czech Republic', International Journal of Hygiene and Environmental Health, Vol. 210, Nos. 3/4, pp.495-499.

Černá, M., Krsková, A., Čejchanová, M. and Spěváčková, V. (2012) 'Human biomonitoring in the Czech Republic: an overview', Int Journal of Hygiene and Environmental Health, Vol. 215, No. 2, pp.109-119.

Chadha, V., Garg, U. and Alon, U.S. (2001) 'Measurement of urinary concentration: a critical appraisal of methodologies', Pediatric Nephrology, Vol. 16, No. 4, pp.374-382.

Chronic Hazard Advisory Panel (CHAP) (2014) Chronichazard Advisory Panel on Phthalate and Phthalate Substitutes, Consumer Product Safety Commission, Bethesda, MD, US [online] http://www.cpsc.gov/PageFiles/169902/CHAP-REPORT-With-Appendices.pdf (accessed 7 January 2016).

Clewell, H.J., Gentry, P.R., Covington, T.R., Sarangapani, R. and Teeguarden, J.G. (2004) 'Evaluation of the potential impact of age-and gender-specific pharmacokinetic differences on tissue dosimetry', Toxicological Sciences, Vol. 79, No. 2, pp.381-393.

Clewell, H.J., Tan, Y.M., Campbell, J.L. and Andersen, M.E. (2008) 'Quantitative interpretation of human biomonitoring data', Toxicology and Applied Pharmacology, Vol. 231, No. 1, pp.122-133.

Cone, E.J., Caplan, Y.H., Moser, F., Robert, T., Shelby, M.K. and Black, D.L. (2009) 'Normalization of urinary drug concentrations with specific gravity and creatinine', $J$ Analytical Toxicology, Vol. 33, No. 1, pp.1-7.

Consumer Product Safety Commission (CPSC) (2001) Chronic Hazard Advisory Panel on Diisononyl Phthalate (DINP), U.S. Consumer Product Safety Commission Directorate for Health Sciences Bethesda, MD 20814 [online] http://www.cpsc.gov/pagefiles/98260/dinp.pdf.

Consumer Products Safety Commission (CPSC) (2015) Estimated Phthalate Exposure and Risk to Pregnant Women and Women of Reproductive Age as Assessed Using Four NHANES Biomonitoring Data Sets (2005/2006, 2007/2008, 2009/2010, 2011/2012), CPSC, Rockville, MD [online] http://www.cpsc.gov/Global/Regulations-Laws-and-Standards/CPSIA/CHAP/ NHANES-Biomonitoring-analysis-for-Commission.pdf (accessed 7 January 2016).

Côté, J., Bonvalot, Y., Carrier, G., Lapointe, C., Fuhr, U., Tomalik-Scharte, D. et al. (2014) A novel toxicokinetic modeling of cypermethrin and permethrin and their metabolites in humans for dose reconstruction from biomarker data', PloS One, Vol. 9, No. 2, p.e88517.

David, R.M. (2000) 'Exposure to phthalate esters', Environmental Health Perspectives, Vol. 108, No. 10, p.A440.

Egeghy, P.P. and Lorber, M. (2011) 'An assessment of the exposure of Americans to perfluorooctane sulfonate: a comparison of estimated intake with values inferred from NHANES data', $J$ Exposure Science and Environmental Epidemiology, Vol. 21, No. 2, pp.150-168.

Environment Canada, Health Canada (2015a) State of the Science Report: Phthalate Substance Grouping: Short-Chain Phthalate Esters: 1,2-Benzenedicarboxylic Acid, Dimethyl Ester (Dimethyl Phthalate (DMP), Environment Canada, Health Canada, Ottawa (ON), on the cover: Chemical Abstracts Service Registry Number: 131-11-3 [online] http://www.ec.gc.ca/ese-ees/default.asp?lang=En\&n=51624E94-1 (accessed 11 December 2015).

Environment Canada, Health Canada (2015b) State of the Science Report: Phthalate Substance Grouping: Medium-Chain Phthalate Esters, Environment Canada, Health Canada, Ottawa, ON, On the cover: Chemical Abstracts Service Registry Numbers: 84-61-7; 84-64-0; 84-69-5; 523-31-9; 5334-09-8; 16883-83-3; 27215-22-1; 27987-25-3; 68515-40-2; 71888-89-6 [online] http://www.ec.gc.ca/ese-ees/default.asp?lang=En\&n=4D845198-1 (accessed 11 December 2015). 
Environment Canada, Health Canada (2015c) State of the Science Report: Phthalate Substance Grouping: 1,2-Benzenedicarboxylic Acid, Diisononyl Ester;1,2-Benzenedicarboxylic Acid, diC8-10-Branched Alkyl Esters, C9-Rich; (Diisononyl Phthalate; DINP), Environment Canada, Health Canada, Ottawa, ON, On the cover: Chemical Abstracts Service Registry Numbers: 28553-12-0 and 68515-48-0 [online] http://www.ec.gc.ca/ese-ees/ default.asp?lang=En\&n=47F58AA5-1 (accessed 11 December 2015).

Environment Canada, Health Canada (2015d) State of the Science Report: Phthalate Substance Grouping: Long-Chain Phthalate Esters: 1,2-Benzenedicarboxylic Acid, Diisodecyl Ester (Diisodecyl Phthalate; DIDP) and 1,2-Benzenedicarboxylic Acid, Diundecyl Ester (Diundecyl Phthalate; DUP), Environment Canada, Health Canada, Ottawa (ON), On the cover: Chemical Abstracts Service Registry Numbers: 26761-40-0; 68515-49-1; 3648-20-2 [online] www.ec.gc.ca/ese-ees/default.asp?lang=En\&n=D3FB0F30-1 (accessed 11 December 2015).

Epstein, M. (1996) 'Aging and the kidney', J American Society of Nephrology, Vol. 7, No. 8, pp.1106-1122.

Eskenazi, B., Bradman, A., Gladstone, E.A., Jaramillo, S., Birch, K. and Holland, N. (2003) 'CHAMACOS, a longitudinal birth cohort study: lessons from the fields', J Children's Health, Vol. 1, No. 1, pp.3-27.

Esteban, M., Schindler, B.K., Jiménez-Guerrero, J.A., Koch, H.M., Angerer, J., Rivas, T.C. et al. (2014) 'Mercury analysis in hair: comparability and quality assessment within the transnational COPHES/DEMOCOPHES project', Environmental Research, Vol. 141, No. 4, pp.24-30.

European Centre for Ecotoxicology and Toxicology of Chemicals (ECETOC) (2005) Guidance for the Interpretation of Biomonitoring Data, Document No. 44.ECETOC, Brussels [online] http://library.wur.nl/WebQuery/clc/1818408 (accessed 7 January 2016).

European Commission (2009) Scientific Committee on Consumer Products (SCCP): Opinion on Triclosan, COLIPA No. P32, European Commission, Burssels [online] http://ec.europa.eu/health/ph_risk/committees/04_sccp/docs/sccp_o_166.pdf (accessed 7 January 2016).

Ewers, U., Krause, C., Schulz, C. and Wilhelm, M. (1999) 'Reference values and human biological monitoring values for environmental toxins', Int Archives Occupational and Environmental Health, Vol. 72, No. 4, pp.255-260.

Fisher, M., Arbuckle, T.E., Mallick, R., LeBlanc, A., Hauser, R., Feeley, M. et al. (2015) 'Bisphenol A and phthalate metabolite urinary concentrations: daily and across pregnancy variability', J Exposure Science and Environmental Epidemiology, Vol. 25, No. 3, pp.231-239.

Frederiksen, H., Aksglaede, L., Sorensen, K., Skakkebaek, N.E., Juul, A. and Andersson, A.M. (2011) 'Urinary excretion of phthalate metabolites in 129 healthy Danish children and adolescents: estimation of daily phthalate intake', Environmental Research, Vol. 111, No. 5, pp.656-663.

Frederiksen, H., Kranich, S.K., Jørgensen, N., Taboureau, O., Petersen, J.H. and Andersson, A.M. (2013) 'Temporal variability in urinary phthalate metabolite excretion based on spot, morning, and 24-h urine samples: considerations for epidemiological studies', Environmental Science and Technology, Vol. 47, No. 2, pp.958-967.

Fréry, N., Saoudi, A., Garnier, R., Zeghnoun, A., Falq, G. and Guldner, L. (2010) Exposure of the French Population to Environmental Pollutants - Environmental Components of the French National Survey on Nutrition and Health - Initial Results, French Institute for Public Health Surveillance Saint-Maurice, Fra [online] http://www.invs.sante.fr/publications/2011/ exposition_polluants_enns/plaquette_eng_exposition_polluants_enns.pdf (accessed 7 January 2016).

Fréry, N., Vandentorren, S., Etchevers, A. and Fillol, C. (2012) 'Highlights of recent studies and future plans for the French human biomonitoring (HBM) programme', Int $J$ Hygiene and Environmental Health, Vol.215, No. 2, pp.127-132. 
Fromme, H., Schlummer, M., Möller, A., Gruber, L., Wolz, G., Ungewiss, J. et al. (2007) 'Exposure of an adult population to perfluorinated substances using duplicate diet portions and biomonitoring data', Environmental Science and Technology, Vol. 41, No. 22, pp.7928-7933.

Fuhr, U., Boettcher, M.I., Kinzig-Schippers, M., Weyer, A., Jetter, A., Lazar, A. et al. (2006) 'Toxicokinetics of acrylamide in humans after ingestion of a defined dose in a test meal to improve risk assessment for acrylamide carcinogenicity', Cancer Epidemiology Biomarkers and Prevention, Vol. 15, No. 2, pp.266-271.

Georgopoulos, P.G., Sasso, A.F., Isukapalli, S.S., Lioy, P.J., Vallero, D.A., Okino, M. and Reiter, L. (2009) 'Reconstructing population exposures to environmental chemicals from biomarkers: challenges and opportunities', $J$ Exposure Science and Environmental Epidemiology, Vol. 19, No. 2, pp.149-171.

Göen, T., Schaller, K.H. and Drexler, H. (2012) 'External quality assessment of human biomonitoring in the range of environmental exposure levels', Int $J$ Hygiene and Environmental Health, Vol. 215, No. 2, pp.229-232.

Government of Canada (2014) Triclosan [online] http://www.chemicalsubstanceschimiques.gc.ca/ plan/approach-approche/triclosan-eng.php (accessed 10 July 2014).

Grandjean, P., Weihe, P., White, R.F., Debes, F., Araki, S., Yokoyama, K. et al. (1997) 'Cognitive deficit in 7-year-old children with prenatal exposure to methylmercury', Neurotoxicology and Teratology, Vol. 19, No. 6, pp.417-428.

Grulke, C.M., Holm, K., Goldsmith, M.R. and Tan, Y.M. (2013) 'PROcEED: probabilistic reverse dosimetry approaches for estimating exposure distributions', Bioinformation, Vol. 9, No. 13, pp.707-709.

Hansen, J.C. (1998) 'The human health programme under AMAP. AMAP human health group. Arctic monitoring and assessment program', Int J Circumpolar Health, Vol. 57, No. 4, pp.280-291.

Hays, S.M. and Aylward, L.L. (2008) 'Biomonitoring equivalents (BE) dossier for acrylamide (AA)(CAS No. 79-06-1)', Regulatory Toxicology and Pharmacology, Vol. 51, No. 3, pp.S57-S67.

Hays, S.M., Aylward, L.L. and Blount, B.C. (2015) 'Variation in urinary flow rates according to demographic characteristics and body mass index in NHANES: potential confounding of associations between health outcomes and urinary biomarker concentrations', Environmental Health Perspectives, Vol. 123, No. 4, pp.293-300.

Hays, S.M., Aylward, L.L., Gagné, M. and Krishnan, K. (2009) 'Derivation of biomonitoring equivalents for cyfluthrin', Regulatory Toxicology and Pharmacology, Vol. 55, No. 3, pp.268-275.

Hays, S.M., Aylward, L.L., Gagné, M., Nong, A. and Krishnan, K. (2010) 'Biomonitoring equivalents for inorganic arsenic', Regulatory Toxicology and Pharmacology, Vol. 58, No. 1, pp.1-9.

Hays, S.M., Aylward, L.L., Kirman, C.R., Krishnan, K. and Nong, A. (2011) 'Biomonitoring equivalents for di-isononyl phthalate (DINP)', Regulatory Toxicology and Pharmacology, Vol. 60, No. 2, pp.181-188.

Hays, S.M., Aylward, L.L., LaKind, J.S., Bartels, M.J., Barton, H.A., Boogaard, P.J. et al. (2008a) 'Guidelines for the derivation of biomonitoring equivalents: report from the biomonitoring equivalents expert workshop', Regulatory Toxicology and Pharmacology, Vol. 51, No. 3, pp.S4-S15.

Hays, S.M., Nordberg, M., Yager, J.W. and Aylward, L.L. (2008b) 'Biomonitoring equivalents (BE) dossier for cadmium (Cd)(CAS No. 7440-43-9)', Regulatory Toxicology and Pharmacology, Vol. 51, No. 3, pp.S49-S56.

Hays, S.M., Becker, R.A., Leung, H.W., Aylward, L.L. and Pyatt, D.W. (2007) 'Biomonitoring equivalents: a screening approach for interpreting biomonitoring results from a public health risk perspective', Regulatory Toxicology and Pharmacology, Vol. 47, No. 1, pp.96-109. 
Hays, S.M., Macey, K., Nong, A. and Aylward, L.L. (2014) 'Biomonitoring equivalents for selenium', Regulatory Toxicology and Pharmacology, Vol. 70, No. 1, pp.333-339.

Health Canada (1995) Approaches to the Derivation of Drinking Water Guidelines, Health Canada, Ottawa, ON [online] http://www.hc-sc.gc.ca/ewh-semt/pubs/water-eau/part_i-partie_i/indexeng.php (accessed 7 January 2016).

Health Canada (1996) Health-Based Tolerable Daily Intakes/Concentrations and Tumorigenic Doses/Concentrations for Priority Substances, Health Canada, Ottawa, ON [online] http://www.hc-sc.gc.ca/ewh-semt/pubs/contaminants/hbct-jact/index-eng.php (accessed 7 January 2016).

Health Canada (2010) Report on Human Biomonitoring of Environmental: Results of the Canadian Health Measures Survey Cycle 1 (2007-2009), Health Canada, Ottawa, ON.

Health Canada (2012) Preliminary Assessment Report on Triclosan, Health Canada and Environment Canada, Ottawa, ON [online] http://www.ec.gc.ca/ese-ees/6EF68BEC-56204435-8729-9B91C57A9FD2/Triclosan_EN.pdf (accessed 7 January 2016).

Health Canada (2013a) Final Human Health State of the Science Report on Lead, Health Canada, Ottawa, ON [online] http://www.hc-sc.gc.ca/ewh-semt/pubs/contaminants/dhhssrl-rpecscepsh/ index-eng.php (accessed 7 January 2016).

Health Canada (2013b) Second Report on Human Biomonitoring of Environmental: Results of the Canadian Health Measures Survey Cycle 2 (2009-2011), Health Canada, Ottawa, ON.

Heffernan, A.L., Aylward, L.L., Toms, L.M.L., Sly, P.D., Macleod, M. and Mueller, J.F. (2014) 'Pooled biological specimens for human biomonitoring of environmental chemicals: opportunities and limitations', J Exposure Science and Environmental Epidemiology, Vol. 24, No. 3, pp.225-232.

Hines, C.J., Hopf, N.B., Deddens, J.A., Silva, M.J. and Calafat, A.M. (2011) 'Estimated daily intake of phthalates in occupationally exposed groups', $J$ Exposure Science and Environmental Epidemiology, Vol. 21, No. 2, pp.133-141.

Horton, M. (2008) 'The California biomonitoring program', Epidemiology, Vol. 19, No. 6, p.S13.

Huang, M.Y., Jin, C., Liu, Y.T., Li, B.H., Qu, Q.S., Uchida, Y. et al. (1994) 'Exposure of workers to a mixture of toluene and xylenes. I. metabolism', Occupational and Environmental Medicine, Vol. 51, No. 1, pp.42-46.

Huber, D.R., Blount, B.C., Mage, D.T., Letkiewicz, F.J., Kumar, A. and Allen, R.H. (2011) 'Estimating perchlorate exposure from food and tap water based on US biomonitoring and occurrence data', J Exposure Science and Environmental Epidemiology, Vol. 21, No. 4, pp.395-407.

Human Biomonitoring (HBM) Commission of the German Federal Environment Agency (2014) 'Grundsatzpapier zur Ableitung von HBM-Werten. Stellungnahme der Kommission HumanBiomonitoring des Umweltbundesamtes', Bundesgesundheitsbl Gesundheitsforsch Gesundheitsschutz, Vol. 57, No. 1, pp.138-147.

Human Biomonitoring (HBM) Commission of the German Federal Environment Agency (2007) 'Derivation of human biomonitoring (HBM) values based on tolerable intake doses. Part II: rationale and way of derivation', Bundesgesundheitsbl. Gesundheitsforsch. Gesundheitsschutz, Vol. 50, No. 2, pp.251-254.

Ikeda, M., Ezaki, T., Tsukahara, T., Moriguchi, J., Furuki, K., Fukui, Y. et al.(2003) 'Bias induced by the use of creatinine-corrected values in evaluation of $\beta$ 2-microgloblin levels', Toxicology Letters, Vol. 145, No. 2, pp.197-207.

Jedrychowski, W., Perera, F., Jankowski, J., Rauh, V., Flak, E., Caldwell, K.L. et al. (2007) 'Fish consumption in pregnancy, cord blood mercury level and cognitive and psychomotor development of infants followed over the first three years of life: Krakow epidemiologic study', Environment International, Vol. 33, No. 8, pp.1057-1062.

Joas, A., Knudsen, L.E., Kolossa-Gehring, M., Sepai, O., Casteleyn, L., Schoeters, G. et al. (2014) 'Policy recommendations and cost implications for a more sustainable framework for European human biomonitoring surveys', Environmental Research, Vol. 141, pp.42-57. 
Joas, R., Casteleyn, L., Biot, P., Kolossa-Gehring, M., Castano, A., Angerer, J. et al. (2012) 'Harmonised human biomonitoring in Europe: activities towards an EU HBM framework', Int J Hygiene and Environmental Health, Vol. 215, No. 2, pp.172-175.

Katsonouri, A., Fischer, M.E., Hadjipanayis, A., Arendt, M., Lavranos, G., Hoffmann, L. et al. (2015) 'Harmonized European human biomonitoring in small countries: challenges, opportunities and lessons learned in Cyprus and Luxembourg from the DEMOCOPHES study', Biomonitoring, Vol. 2, No. 1, pp.21-34.

Kim, Y. (2014) 'The Korea National Health And Nutrition Examination Survey (KNHANES): current status and challenges', Epidemiology and Health, Vol. 36, p.e2014002.

Kirman, C.R., Aylward, L.L., Hays, S.M., Krishnan, K. and Nong, A. (2011) 'Biomonitoring equivalents for DDT/DDE', Regulatory Toxicology and Pharmacology, Vol. 60, No. 2, pp.172-180.

Kirman, C.R., Sweeney, L.M., Meek, M.E. and Gargas, M.L. (2003) 'Assessing the dosedependency of allometric scaling performance using physiologically based pharmacokinetic modeling', Regulatory Toxicology and Pharmacology, Vol. 38, No. 3, pp.345-367.

Kissel, J.C., Curl, C.L., Kedan, G., Lu, C., Griffith, W., Barr, D.B. et al.(2005) 'Comparison of organophosphorus pesticide metabolite levels in single and multiple daily urine samples collected from preschool children in Washington State', $J$ Exposure Science and Environmental Epidemiology, Vol. 15, No. 2, pp.164-171.

Kjellström, T., Kennedy, P., Wallis, S. and Mantell, C. (1986) Physical and Mental Development of Children with Prenatal Exposure to Mercury from Fish. Stage 1: Preliminary Tests at Age 4, National Swedish Environmental Protection Board, Solna, Sweden.

Kjellström, T., Kennedy, P., Wallis, S., Stewart, A., Friberg, L., Lind, B., Wutherspoon, T. and Mantell, C. (1989) Physical and Mental Development of Children with Prenatal Exposure to Mercury from Fish. Stage 2. Interviews and Psychological Tests at Age 6, National Swedish Environmental Protection Board, Solna, Sweden.

Koch, H.M. and Angerer, J. (2007) 'Di-iso-nonylphthalate (DINP) metabolites in human urine after a single oral dose of deuterium-labelled DINP', Int $J$ Hygiene and Environmental Health, Vol. 210, No. 1, pp.9-19.

Koch, H.M. and Calafat, A.M. (2009) 'Human body burdens of chemicals used in plastic manufacture', Philosophical Transactions Royal Society B: Biological Sciences, Vol. 364, No. 1526, pp.2063-2078.

Koch, H.M., Aylward, L.L., Hays, S.M., Smolders, R., Moos, R.K., Cocker, J. et al. (2014) 'Inter-and intra-individual variation in urinary biomarker concentrations over a 6-day sampling period. Part 2: personal care product ingredients', Toxicology Letters, Vol. 231, No. 2, pp.261-269.

Koch, H.M., Bolt, H.M., Preuss, R. and Angerer, J. (2005) 'New metabolites of di (2-ethylhexyl) phthalate (DEHP) in human urine and serum after single oral doses of deuterium-labelled DEHP', Archives of Toxicology, Vol. 79, No. 7, pp.367-376.

Koch, H.M., Drexler, H. and Angerer, J. (2003) 'An estimation of the daily intake of di (2-ethylhexyl) phthalate (DEHP) and other phthalates in the general population', Int J Hygiene and Environmental Health, Vol. 206, No. 2, pp.77-83.

Kohn, M.C., Parham, F., Masten, S.A., Portier, C.J., Shelby, M.D., Brock, J.W. and Needham, L.L. (2000) 'Human exposure estimates for phthalates', Environmental Health Perspectives, Vol. 108, No. 10, pp.A440-A442.

Krewski, D., Westphal, M., Andersen, M.E., Paoli, G.M., Chiu, W.A., Al-Zoughool, M. et al. (2014) 'A framework for the next generation of risk science', Environmental Health Perspectives, Vol. 122, No. 8, pp.796-805.

Krishnan, K., Adamou, T., Aylward, L.L., Hays, S.M., Kirman, C.R. and Nong, A. (2011) 'Biomonitoring equivalents for 2, 2, 4, 4', 5-pentabromodiphenylether (PBDE-99)', Regulatory Toxicology and Pharmacology, Vol. 60, No. 2, pp.165-171. 
Krishnan, K., Gagné, M., Nong, A., Aylward, L.L. and Hays, S.M. (2010a) 'Biomonitoring equivalents for bisphenol A (BPA)', Regulatory Toxicology and Pharmacology, Vol. 58, No. 1, pp.18-24.

Krishnan, K., Gagné, M., Nong, A., Aylward, L.L. and Hays, S.M. (2010b) 'Biomonitoring equivalents for triclosan', Regulatory Toxicology and Pharmacology, Vol. 58, No. 1, pp.10-17.

Kroes, R., Kleiner, J. and Renwick, A. (2005) 'The threshold of toxicological concern concept in risk assessment', Toxicological Sciences, Vol. 86, No. 2, pp.226-230.

Kweon, S., Kim, Y., Jang, M.J., Kim, Y., Kim, K., Choi, S. et al. (2014) 'Data resource profile: the Korea National Health And Nutrition Examination Survey (KNHANES)', Int J Epidemiology, Vol. 43, No. 1, pp.69-77.

La Corte, E. and Wuttke, S. (2012) 'The First Nations Biomonitoring Initiative - FNBI', Int J Hygiene and Environmental Health, Vol. 215, No. 2, pp.168-171.

Lakind, J.S. and Naiman, D.Q. (2008) 'Bisphenol A (BPA) daily intakes in the United States: estimates from the 2003-2004 NHANES urinary BPA data', Journal of Exposure Science and Environmental Epidemiology, Vol. 18, No. 6, pp.608-615.

LaKind, J.S. and Naiman, D.Q. (2011) 'Daily intake of bisphenol A and potential sources of exposure: 2005-2006 National Health And Nutrition Examination Survey', J Exposure Science and Environmental Epidemiology, Vol. 21, No. 3, pp.272-279.

LaKind, J.S., Aylward, L.L., Brunk, C., DiZio, S., Dourson, M., Goldstein, D.A. et al. (2008) 'Guidelines for the communication of biomonitoring equivalents: report from the biomonitoring equivalents expert workshop', Regulatory Toxicology and Pharmacology, Vol. 51, No. 3, pp.S16-S26.

Landrigan, P.J., Trasande, L., Thorpe, L.E., Gwynn, C., Lioy, P.J., D’Alton, M.E. et al. (2006) 'The national children's study: a 21-year prospective study of 100,000 American children', Pediatrics, Vol. 118, No. 5, pp.2173-2186.

Langlois, É., Saravanabhavan, G., Arbuckle, T.E. and Giroux, S. (2014) 'Correction and comparability of phthalate metabolite measurements of Canadian biomonitoring studies (2007-2012)', Environment Int, Vol. 64, pp.129-133.

Lanphear, B.P. (2005) 'Origins and evolution of children's environmental health', in Goehl, T.J. (Eds.) In Essays on the Future of Environmental Health Research: A Tribute to Kenneth Olden, Special Issue, pp.24-31, National Institute of Environmental Health Sciences, Research Triangle Park.

Lanphear, B.P., Hornung, R., Khoury, J., Yolton, K., Baghurst, P., Bellinger, D.C. et al. (2005) 'Low-level environmental lead exposure and children's intellectual function: an international pooled analysis', Environmental Health Perspectives, Vol. 113, No. 7, pp.894-899.

Leng, G., Leng, A., Kuhn, K.H. and Lewalter, J. (1997) 'Human dose-excretion studies with the pyrethroid insecticide cyfluthrin: urinary metabolite profile following inhalation', Xenobiotica, Vol. 27, No. 12, pp.1273-1283.

Lentner, C. (1981) Geigy Scientific Tables. Vol. 1, Units of Measurement, Body Fluids, Composition of the Body, Nutrition, Ciba-Geigy, Basle.

Li, Z., Romanoff, L.C., Lewin, M.D., Porter, E.N., Trinidad, D.A., Needham, L.L. et al. (2010) 'Variability of urinary concentrations of polycyclic aromatic hydrocarbon metabolite in general population and comparison of spot, first-morning, and 24-h void sampling', $J$ Exposure Science and Environmental Epidemiology, Vol. 20, No. 6, pp.526-535.

Lioy, P.J., Hauser, R., Gennings, C., Koch, H.M., Mirkes, P.E., Schwetz, B.A. and Kortenkamp, A. (2015) 'Assessment of phthalates/phthalate alternatives in children's toys and childcare articles: review of the report including conclusions and recommendation of the chronic hazard advisory panel of the Consumer Product Safety Commission', J Exposure Science and Environmental Epidemiology, Vol. 25, No. 4, pp.343-353. 
Lorber, M. and Egeghy, P.P. (2011) 'Simple intake and pharmacokinetic modeling to characterize exposure of Americans to perfluoroctanoic acid, PFOA', Environmental Science and Technology, Vol. 45, No. 19, pp.8006-8014.

Lowry, L.K. (1986) 'Biological exposure index as a complement to the TLV', J Occupational Medicine, Vol. 28, No. 8, pp.578-582.

Lu, F.C. (1988) 'Acceptable daily intake: inception, evolution, and application', Regulatory Toxicology and Pharmacology, Vol. 8, No. 1, pp.45-60.

Lyons, M.A., Yang, R.S., Mayeno, A.N. and Reisfeld, B. (2008) 'Computational toxicology of chloroform: reverse dosimetry using Bayesian inference, Markov chain Monte Carlo simulation, and human biomonitoring data', Environmental Health Perspectives, Vol. 116, No. 8, pp.1040-1046.

Mage, D.T., Allen, R.H. and Kodali, A. (2008) 'Creatinine corrections for estimating children's and adult's pesticide intake doses in equilibrium with urinary pesticide and creatinine concentrations', Journal of Exposure Science and Environmental Epidemiology, Vol. 18, No. 4, pp.360-368.

Mage, D.T., Allen, R.H., Gondy, G., Smith, W., Barr, D.B. and Needham, L.L. (2004) 'Estimating pesticide dose from urinary pesticide concentration data by creatinine correction in the third National Health And Nutrition Examination Survey (NHANES-III)', J Exposure Science and Environmental Epidemiology, Vol. 14, No. 6, pp.457-465.

Magnusson, B. and Örnemark, U. (Eds.) (2014) Eurachem Guide: The Fitness for Purpose of Analytical Methods - A Laboratory Guide to Method Validation and Related Topics, 2nd ed. [online] http://www.Eurachem.Org (accessed 7 January 2016).

Mahalingaiah, S., Meeker, J.D., Pearson, K.R., Calafat, A.M., Ye, X., Petrozza, J. and Hauser, R. (2008) 'Temporal variability and predictors of urinary bisphenol A concentrations in men and women', Environmental Health Perspectives, Vol. 116, No. 2, pp.173-178.

Maier, A., Savage, R.E. and Haber, L.T. (2004) 'Assessing biomarker use in risk assessment - a survey of practitioners', $J$ Toxicology and Environmental Health, Part A, Vol. 67, Nos. 8-10, pp.687-695.

McDonald, T.A., Holland, N.T., Skibola, C., Duramad, P. and Smith, M.T. (2001) 'Hypothesis: phenol and hydroquinone derived mainly from diet and gastrointestinal flora activity are causal factors in leukemia', Leukemia, Vol. 15, No. 1, pp.10-20.

McNally, K., Cotton, R., Cocker, J., Jones, K., Bartels, M., Rick, D. et al. (2012) 'Reconstruction of exposure to m-Xylene from human biomonitoring data using PBPK modelling, Bayesian inference, and Markov chain Monte Carlo simulation', J Toxicology, Article ID 760281.

Miller, R.C., Brindle, E., Holman, D.J., Shofer, J., Klein, N.A., Soules, M.R. and O’Connor, K.A. (2004) 'Comparison of specific gravity and creatinine for normalizing urinary reproductive hormone concentrations', Clinical Chemistry, Vol. 50, No. 5, pp.924-932.

Mühlberg, W. and Platt, D. (1999) 'Age-dependent changes of the kidneys: pharmacological implications', Gerontology, Vol. 45, No. 5, pp.243-253.

National Research Council (NRC) (2006) Human Biomonitoring for Environmental Chemicals.Committee on Human Biomonitoring for Environmental Toxicants, National Research Council, Washington, DC.

National Research Council (NRC) (2012) 'Exposure science in the 21st century: a vision and a strategy', Edited by Committee on Human and Environmental Exposure Science in the 21st Century, Board on Environmental Studies and Toxicology; Division on Earth and Life Studies, National Research Council, Washington, DC.

Needham, L.L., Calafat, A.M. and Barr, D.B. (2007) 'Uses and issues of biomonitoring', Int Hygiene and Environmental Health, Vol. 210, Nos. 3-4, pp.229-238.

Padmanabhan, V., Siefert, K., Ransom, S., Johnson, T., Pinkerton, J., Anderson, L. et al. (2008) 'Maternal bisphenol-A levels at delivery: a looming problem?', J Perinatology, Vol. 28, No. 4, pp.258-263. 
Pearson, M.A., Lu, C., Schmotzer, B.J., Waller, L.A. and Riederer, A.M. (2009) 'Evaluation of physiological measures for correcting variation in urinary output: implications for assessing environmental chemical exposure in children', $J$ Exposure Science and Environmental Epidemiology, Vol. 19, No. 3, pp.336-342.

Pirkle, J.L., Brody, D.J., Gunter, E.W., Kramer, R.A., Paschal, D.C., Flegal, K.M. and Matte, T.D. (1994) 'The decline in blood lead levels in the United States: the National Health And Nutrition Examination Surveys (NHANES)', The Journal of the American Medical Association, Vol. 272, No. 4, pp.284-291.

Preau, J.L., Wong, L.Y., Silva, M.J., Needham, L.L. and Calafat, A.M. (2010) 'Variability over 1 week in the urinary concentrations of metabolites of diethyl phthalate and di (2-ethylhexyl) phthalate among eight adults: an observational study', Environmental Health Perspectives, Vol. 118, No. 12, pp.1748-1754.

Rawn, D.F., Ryan, J.J., Sadler, A.R., Sun, W.F., Haines, D., Macey, K. and van Oostdam, J. (2012) 'PCDD/F and PCB concentrations in sera from the Canadian Health Measures Survey (CHMS) from 2007 to 2009', Environment Int, Vol. 47, pp.48-55.

Remer, T., Neubert, A. and Maser-Gluth, C. (2002) 'Anthropometry-based reference values for 24-h urinary creatinine excretion during growth and their use in endocrine and nutritional research', The American Journal of Clinical Nutrition, Vol. 75, No. 3, pp.561-569.

Sand, S., Portier, C.J. and Krewski, D. (2011) 'A signal-to-noise crossover dose as the point of departure for health risk assessment', Environmental Health Perspectives, Vol. 119, No. 12, pp.1766.

Sandborgh-Englund, G., Adolfsson-Erici, M., Odham, G. and Ekstrand, J. (2006) 'Pharmacokinetics of triclosan following oral ingestion in humans', Journal of Toxicology and Environmental Health, Part A, Vol. 69, No. 20, pp.1861-1873.

Saravanabhavan, G., Guay, M., Langlois, É., Giroux, S., Murray, J. and Haines, D. (2013) 'Biomonitoring of phthalate metabolites in the Canadian population through the Canadian Health Measures Survey (2007-2009)', Int J Hygiene and Environmental Health, Vol. 216, No. 6, pp.652-661.

Saravanabhavan, G., Walker, M., Guay, M. and Aylward, L. (2014) 'Urinary excretion and daily intake rates of diethyl phthalate in the general Canadian population', Science of the Total Environment, Vol. 500, pp.191-198.

Schindler, B.K., Esteban, M., Koch, H.M., Castano, A., Koslitz, S., Cañas, A. et al. (2014) 'The European COPHES/DEMOCOPHES project: towards transnational comparability and reliability of human biomonitoring results', Int J Hygiene and Environmental Health, Vol. 217, No. 6, pp.653-661.

Schisterman, E.F., Perkins, N.J., Liu, A. and Bondell, H. (2005) 'Optimal cut-point and its corresponding Youden index to discriminate individuals using pooled blood samples', Epidemiology, Vol. 16, No. 1, pp.73-81.

Schoeters, G., Den Hond, E., Colles, A., Loots, I., Morrens, B., Keune, H. et al. (2012) 'Concept of the Flemish human biomonitoring programme', Int J Hygiene and Environmental Health, Vol. 215, No. 2, pp.102-108.

Schroijen, C., Baeyens, W., Schoeters, G., Den Hond, E., Koppen, G., Bruckers, L. et al. (2008) 'Internal exposure to pollutants measured in blood and urine of Flemish adolescents in function of area of residence', Chemosphere, Vol. 71, No. 7, pp.1317-1325.

Schulz, C., Angerer, J., Ewers, U. and Kolossa-Gehring, M. (2007a) 'The German human biomonitoring commission', Int J Hygiene and Environmental Health, Vol. 210, Nos. 3-4, pp.373-382.

Schulz, C., Conrad, A., Becker, K., Kolossa-Gehring, M., Seiwert, M. and Seifert, B. (2007b) 'Twenty years of the German Environmental Survey (GerES): human biomonitoring temporal and spatial (West Germany/East Germany) differences in population exposure', Int J Hygiene and Environmental Health, Vol. 210, Nos. 3-4, pp.271-297. 
Schulz, C., Wilhelm, M., Heudorf, U. and Kolossa-Gehring, M. (2011) 'Update of the reference and HBM values derived by the German Human Biomonitoring Commission', Int J Hygiene and Environmental Health, Vol. 215, No. 2, pp.26-35.

Seifert, B.E.R.N.D., Becker, K., Hoffmann, K., Krause, C. and Schulz, C. (1999) 'The German Environmental Survey 1990/1992 (GerES II): a representative population study', J Exposure Science and Environmental Epidemiology, Vol. 10, No. 2, pp.103-114.

Sexton, K., Needham, L. and Pirkle, J. (2004) 'Human biomonitoring of environmental chemicals measuring chemicals in human tissues is the 'gold standard' for assessing people's exposure to pollution', American Scientist, Vol. 92, pp.38-45.

Silva, M.J., Samandar, E., Preau, J.L., Needham, L.L. and Calafat, A.M. (2006) 'Urinary oxidative metabolites of di (2-ethylhexyl) phthalate in humans', Toxicology, Vol. 219, Nos. 1-3, pp.22-32.

Smolders, R., Den Hond, E., Koppen, G., Govarts, E., Willems, H., Casteleyn, L. et al. (2014) 'Interpreting biomarker data from the COPHES/DEMOCOPHES twin projects: using external exposure data to understand biomarker differences among countries', Environmental Research, Vol. 141, pp.86-95.

Smolders, R., Schramm, K.W., Stenius, U., Grellier, J., Kahn, A., Trnovec, T. et al. (2009) 'A review on the practical application of human biomonitoring in integrated environmental health impact assessment', $J$ Toxicology and Environmental Health, Part B, Vol. 12, No. 2, pp.107-123.

Spaan, S., Fransman, W., Warren, N., Cotton, R., Cocker, J. and Tielemans, E. (2010) 'Variability of biomarkers in volunteer studies: the biological component', Toxicology Letters, Vol. 198, No. 2, pp.144-151.

St-Amand, A., Werry, K., Aylward, L.L., Hays, S.M. and Nong, A. (2014) 'Screening of population level biomonitoring data from the Canadian Health Measures Survey in a riskbased context', Toxicology Letters, Vol. 231, No. 2, pp.126-134.

Tan, Y.M. and Clewell, H.J. (2010) 'Probabilistic reverse dosimetry modeling for interpreting biomonitoring data', in Krishnan, K. and Andersen, M.E. (Eds.): Quantitative Modeling in Toxicology, Wiltshire, Great Britain, pp.353-369.

Tan, Y.M., Liao, K.H. and Clewell, H.J. (2007) 'Reverse dosimetry: interpreting trihalomethanes biomonitoring data using physiologically based pharmacokinetic modeling', $J$ Exposure Science and Environmental Epidemiology, Vol. 17, No. 7, pp.591-603.

Tan, Y.M., Sobus, J., Chang, D., Tornero-Velez, R., Goldsmith, M., Pleil, J. and Dary, C. (2012) 'Reconstructing human exposures using biomarkers and other 'clues', $J$ Toxicology and Environmental Health, Part B, Vol. 15, No. 1, pp.22-38.

Tardif, R., Droz, P.O., Charest-Tardif, G., Pierrehumbert, G. and Truchon, G. (2002) 'Impact of human variability on the biological monitoring of exposure to toluene: I. Physiologically based toxicokinetic modelling', Toxicology Letters, Vol. 134, Nos. 1/3, pp.155-163.

Thompson, J., Lorber, M., Toms, L.M.L., Kato, K., Calafat, A.M. and Mueller, J.F. (2010) 'Use of simple pharmacokinetic modeling to characterize exposure of Australians to perfluorooctanoic acid and perfluorooctane sulfonic acid', Environment International, Vol. 36, No. 4, pp.390-397.

Trasande, L., Landrigan, P.J. and Schechter, C. (2005) 'Public health and economic consequences of methyl mercury toxicity to the developing brain', Environmental Health Perspectives, Vol. 113, No. 5, pp.590-596.

Trudel, D., Horowitz, L., Wormuth, M., Scheringer, M., Cousins, I.T. and Hungerbühler, K. (2008) 'Estimating consumer exposure to PFOS and PFOA', Risk Analysis, Vol. 28, No. 2, pp.251-269.

Ukai, H., Takada, S., Inui, S., Imai, Y., Kawai, T., Shimbo, S.I. and Ikeda, M. (1994) 'Occupational exposure to solvent mixtures: effects on health and metabolism', Occupational and Environmental Medicine, Vol. 51, No. 8, pp.523-529. 
United States Environmental Protection Agency (USEPA) (2005) Human Health Risk Assessment Protocol for Hazardous Waste Combustion Facilities, Multimedia Planning and Permitting Division, Center for Combustion Science and Engineering, Office of Solid Waste and Emergency Response, USEPA [online] http://www3.epa.gov/epawaste/hazard/tsd/td/combust/ riskvol.htm (accessed 7 January 2016).

United States Environmental Protection Agency (USEPA) (2008) Reregistration Eligibility Document (RED) for Triclosan, USEPA [online] http://nepis.epa.gov/Exe/ZyNET.exe/ P1001QGB.TXT?ZyActionD=ZyDocument\&Client=EPA\&Index=2006+Thru+2010\&Docs= $\&$ Query $=\&$ Time $=\&$ EndTime $=\&$ SearchMethod $=1 \&$ TocRestrict $=n \&$ Toc $=\&$ TocEntry $=\& Q F i e l$ $\mathrm{d}=\& Q$ Field Year $=\& Q$ FieldMonth $=\& Q$ FieldDay $=\&$ IntQFieldOp $=0 \&$ ExtQFieldOp $=0 \& X m l Q u$ ery $=\&$ File $=$ D $\% 3 \mathrm{~A} \% 5 \mathrm{Czy}$ files $\% 5 \mathrm{CIndex} \% 20 \mathrm{Data} \% 5 \mathrm{C} 06$ thru $10 \% 5 \mathrm{CTxt} \% 5 \mathrm{C} 00000004 \% 5 \mathrm{CP}$ 1001QGB.txt\&User $=$ ANONYMOUS\&Password=anonymous\&SortMethod $=\mathrm{h} \% 7 \mathrm{C}-$ \&MaximumDocuments $=1 \&$ FuzzyDegree $=0$ \&ImageQuality $=\mathrm{r} 75 \mathrm{~g} 8 / \mathrm{r} 75 \mathrm{~g} 8 / \mathrm{x} 150 \mathrm{y} 150 \mathrm{~g} 16 / \mathrm{i} 425$ $\&$ Display $=\mathrm{p} \%$ 7Cf $\&$ DefSeekPage $=\mathrm{x} \&$ SearchBack $=$ ZyActionL\&Back=ZyActionS\&BackDesc $=$ Results $\% 20$ page $\&$ MaximumPages $=1 \& Z y$ Entry $=1 \&$ SeekPage $=x \& Z y P U R L($ accessed 7 January 2016).

United States Environmental Protection Agency (USEPA) (2011) Memorandum. 2,4-D Petition for the Establishment of a New Formulation of 2,4-D Choline on Herbicide Tolerant Field Corn Containing the Aryloxyalkonate Dioxygenase-1 (ADD-1) Gene, October 27.

United States Environmental Protection Agency (USEPA) (2012) Integrated Risk Information System (IRIS) Database [online] $\mathrm{https}$ ://cfpub.epa.gov/ncea/iris/search/index.cfm?keyword=exposure+guidance+value (accessed 15 September 2012).

United States Environmental Protection Agency (USEPA) (2015) Integrated Risk Information System (IRIS), USEPA [online] http://www.epa.gov/IRIS/ (accessed 7 January 2015).

Valentin, J. (2002) 'Basic anatomical and physiological data for use in radiological protection: reference values: ICRP Publication 89', Annals of ICRP, Vol. 32, No. 3, pp.1-277.

Vandenberg, L.N., Gerona, R.R., Kannan, K., Taylor, J.A., van Breemen, R.B., Dickenson, C.A. et al. (2014) 'A round robin approach to the analysis of bisphenol a (BPA) in human blood samples', Environmental Health, Vol. 13, No. 1, pp.25-44.

Verret, F. and Giroux, S. (2010) La Formation De Pools De Serum De Sang Pour l'Analyse Dans l'Enquête Canadienne Sur Les Mesures De La Santé, Statistical Society of Canada, Ottawa, ON [online] http://ssc.ca/webfm_send/531 (accessed 7 January 2016).

Wittassek, M., Heger, W., Koch, H.M., Becker, K., Angerer, J. and Kolossa-Gehring, M. (2007a) 'Daily intake of di (2-ethylhexyl) phthalate (DEHP) by German children - a comparison of two estimation models based on urinary DEHP metabolite levels', Int J Hygiene and Environmental Health, Vol. 210, No. 1, pp.35-42.

Wittassek, M., Wiesmüller, G.A., Koch, H.M., Eckard, R., Dobler, L., Müller, J. et al. (2007b) 'Internal phthalate exposure over the last two decades-a retrospective human biomonitoring study', Int J Hygiene and Environmental Health, Ann ICRP, Vol. 210, Nos. 3/4, pp.319-333.

World Health Organization (WHO) (2001) Environmental Health Criteria 222: Biomarkers in Risk Assessment: Validity and Validation, WHO, Geneva [online] http://www.inchem.org/ documents/ehc/ehc/ehc222.htm (accessed 7 January 2016).

Wuttke, S., LaCorte, E., Garcia, D. and Ooi, M. (2013) First Nations Biomonitoring Initiative: National Results (2011), Assembly of First Nations, Ottawa, ON [online] http://www.afn.ca/ uploads/files/afn_fnbi_en_-_2013-06-26.pdf (accessed 7 January 2016).

Ye, X., Wong, L.Y., Bishop, A.M. and Calafat, A.M. (2011) 'Variability of urinary concentrations of bisphenol A in spot samples, first morning voids, and 24-hour collections', Environmental Health Perspectives, Vol. 119, No. 7, pp.983-988. 
Ye, X., Zhou, X., Hennings, R., Kramer, J. and Calafat, A.M. (2013) 'Potential external contamination with bisphenol A and other ubiquitous organic environmental chemicals during biomonitoring analysis: an elusive laboratory challenge', Environmental Health Perspectives, Vol. 121, No. 3, pp.283-286.

Ye, X., Zhou, X., Wong, L.Y. and Calafat, A.M. (2012) 'Concentrations of bisphenol A and seven other phenols in pooled sera from 3-11 year old children: 2001-2002 National Health and Nutrition Examination Survey', Environmental Science and Technology, Vol. 46, No. 22, pp.12664-12671. 